\title{
Naphtho[2,1-e]-1,2-azaphosphorine-2-oxide Derivatives: Synthesis, Optoelectronic Properties, and Self-Dimerization Phenomena
}

Chun-Lin Deng, ${ }^{\dagger}$ Jeremy P. Bard,${ }^{\dagger}$ Lev N. Zakharov, ${ }^{\ddagger}$ Darren W. Johnson, ${ }^{*}, \dagger$ and Michael M. Haley*,†

Department of Chemistry \& Biochemistry and the Materials Science Institute, University of Oregon, Eugene, Oregon 97403-1253, United States

¿AMCOR, University of Oregon, Eugene, Oregon 97403-1433, United States

1. X-ray Structure Data and Molecular Packing

2. Photophysical Properties

3. Theoretical Calculations

3.1 TD-DFT Calculations and Cartesian Coordinates

3.2 Dimerization Computations

4. Measurements of Dimerization Constants $K_{\text {dim }}$

5. References

6. Copies of NMR Spectra for New Compounds 


\section{X-ray Structure Data and Molecular Packing}

Diffraction intensities for 8c and 8d were collected at $173 \mathrm{~K}$ on a Bruker Apex2 CCD diffractometer using $\mathrm{CuK} \alpha$ radiation, $\lambda=1.54178 \AA$. Space groups were determined based on intensity statistics. Absorption corrections were applied by SADABS. ${ }^{1}$ Structures were solved by direct methods and Fourier techniques and refined on $F^{2}$ using full matrix least-squares procedures. All non-H atoms were refined with anisotropic thermal parameters. All $\mathrm{H}$ atoms in $\mathbf{8 d}$ and $\mathrm{H}$ atoms at the $\mathrm{N}$ atoms in $\mathbf{8 c}$ involved in $\mathrm{H}$-bonds were found on the residual density maps and refined with isotropic thermal parameters. Other $\mathrm{H}$ atoms in $8 \mathbf{c}$ were refined in calculated positions in a rigid group model. Solvent molecules $\mathrm{CHCl}_{3}$ in $\mathbf{8 c}$ fill out in the packing a space between main molecules and its positions appear to be partially occupied or highly disordered. As a result diffraction intensities at high angles are very weak. These solvents molecules on $\mathbf{8 c}$ were treated by SQUEEZE. ${ }^{2}$ The correction of the X-ray data by SQUEEZE is 228 electron/cell; the required value is 232 electron/cell for four $\mathrm{CHCl}_{3}$ molecules in the full unit cell. All calculations were performed by the Bruker SHELXL-2014 package. ${ }^{3}$ The ORTEP drawings were made with the Diamond ${ }^{4}$ and Ortep- $3^{5}$ programs. The visualization and exploration of the intermolecular close contacts of the structure was achieved by the Hirshfeld surface analysis with the Crystal Explorer software. ${ }^{6}$ CCDC 1900247 and 1900248 contain the supplementary crystallographic data for this paper. These data can be obtained free of charge from The Cambridge Crystallographic Data Centre at www.ccdc.cam.ac.uk/data_request/cif.

Crystallographic data for 8c: $\mathrm{C}_{25} \mathrm{H}_{17} \mathrm{~N}_{2} \mathrm{O}_{2} \mathrm{P} \cdot \mathrm{CHCl}_{3}, \mathrm{M}=527.74,0.11 \times 0.03 \times 0.02 \mathrm{~mm}$, $\mathrm{T}$ $=173(2) \mathrm{K}$, Triclinic, space group $P-1, a=12.7196(6) \AA, b=14.8501(7) \AA, c=15.4304(7) \AA, \alpha$ $=117.135(3)^{\circ}, \beta=106.977(3)^{\circ}, \gamma=94.402(3)^{\circ}, V=2404.1(2) \AA^{3}, Z=4, D c=1.458 \mathrm{Mg} \mathrm{m}^{-3}$,

$\mu(\mathrm{Cu})=4.310 \mathrm{~mm}^{-1}, \mathrm{~F}(000)=1080,2 \theta_{\max }=133.44^{\circ}, 30824$ reflections, 8446 independent reflections $\left[\mathrm{R}_{\text {int }}=0.0546\right], \mathrm{R} 1=0.1027, \mathrm{wR} 2=0.2856$ and $\mathrm{GOF}=1.115$ for 8446 reflections (549 parameters) with $\mathrm{I}>2 \sigma(\mathrm{I}), \mathrm{R} 1=0.1241$, wR $2=0.3052$ and $\mathrm{GOF}=1.119$ for all reflections, $\max / \min$ residual electron density $+1.335 /-0.332 \mathrm{e}^{-3}$. CCDC 1900247.

Crystallographic data for 8d: $\mathrm{C}_{25} \mathrm{H}_{20} \mathrm{NO}_{4} \mathrm{PS}, \mathrm{M}=461.45,0.12 \times 0.09 \times 0.07 \mathrm{~mm}, \mathrm{~T}=$ 173(2) K, Triclinic, space group $P-1, a=8.3012(3) \AA, b=11.3430(4) \AA, c=13.0055(5) \AA, \alpha=$ $93.515(2)^{\circ}, \beta=103.769(2)^{\circ}, \gamma=110.518(2)^{\circ}, V=1099.77(7) \AA^{3}, Z=2, D_{\mathrm{c}}=1.393 \mathrm{Mg} \mathrm{m}^{-3}$, 
$\mu(\mathrm{Cu})=2.273 \mathrm{~mm}^{-1}, F(000)=480,2 \theta_{\max }=133.10^{\circ}, 12225$ reflections, 3871 independent reflections $\left[\mathrm{R}_{\text {int }}=0.0453\right], \mathrm{R} 1=0.0370, \mathrm{wR} 2=0.0993$ and $\mathrm{GOF}=1.029$ for 3871 reflections (369 parameters) with $\mathrm{I}>2 \sigma(\mathrm{I}), \mathrm{R} 1=0.0424, \mathrm{wR} 2=0.1039$ and $\mathrm{GOF}=1.029$ for all reflections, $\max /$ min residual electron density $+0.399 /-0.342 \mathrm{e}^{-3}$. CCDC 1900248.

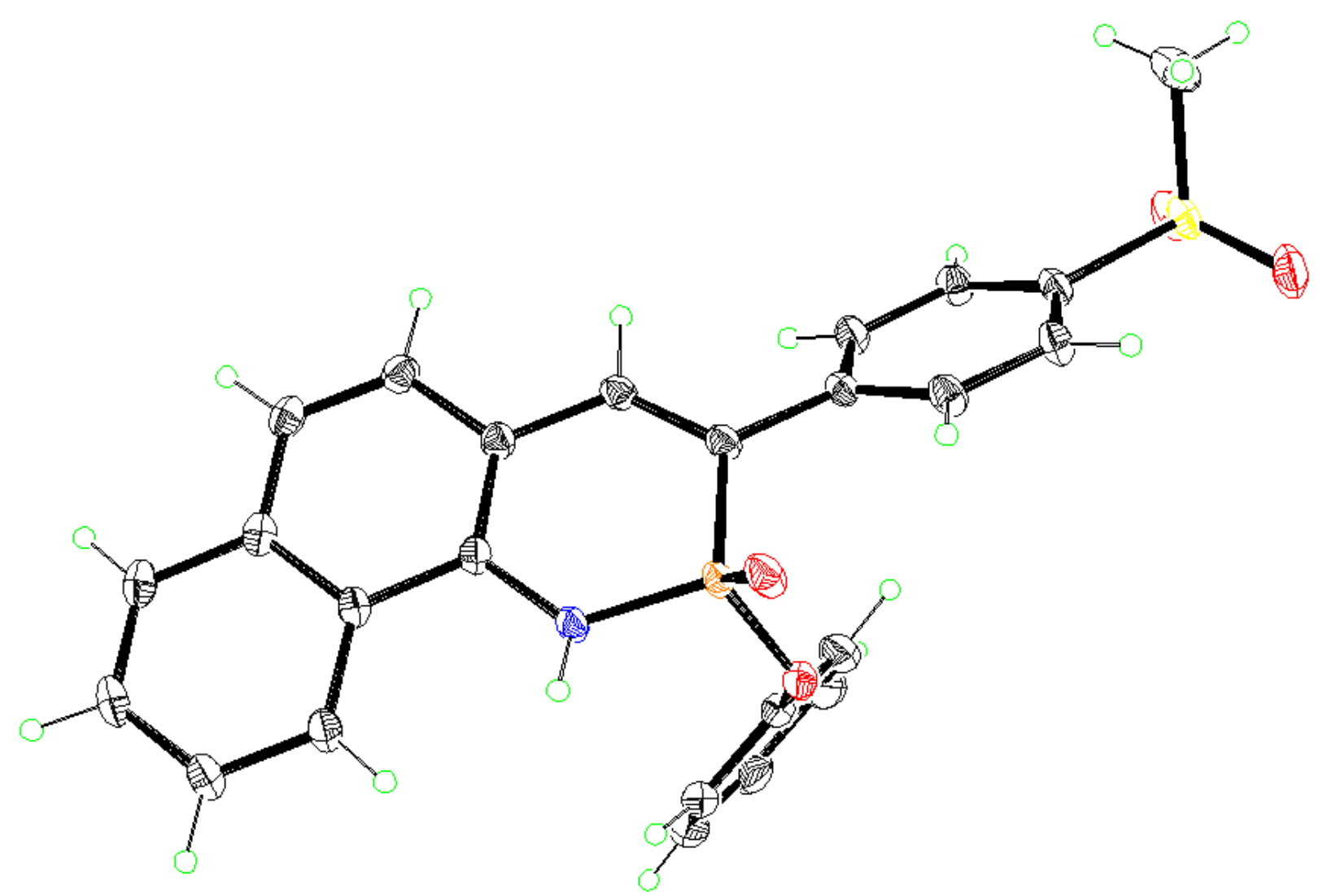

Figure S1. ORTEP drawing of the structure of 8d (thermal ellipsoids drawn at 30\% probability). Single crystals suitable for X-ray diffraction were obtained by slow evaporation of $\mathrm{CH}_{2} \mathrm{Cl}_{2}$ solution at room temperature. 
(a)

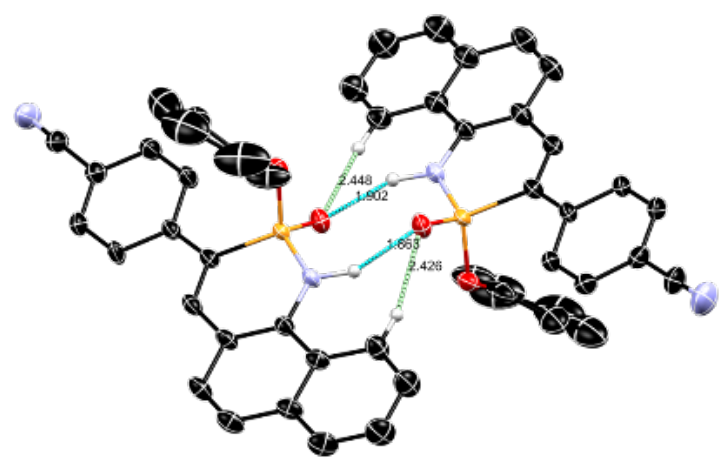

(b)

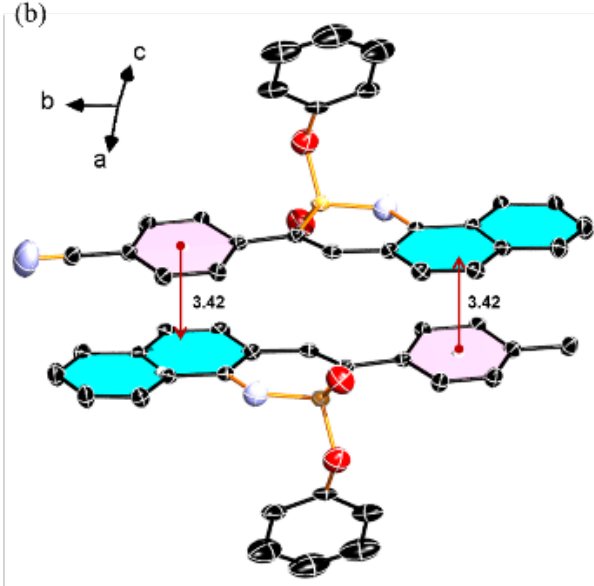

(c)

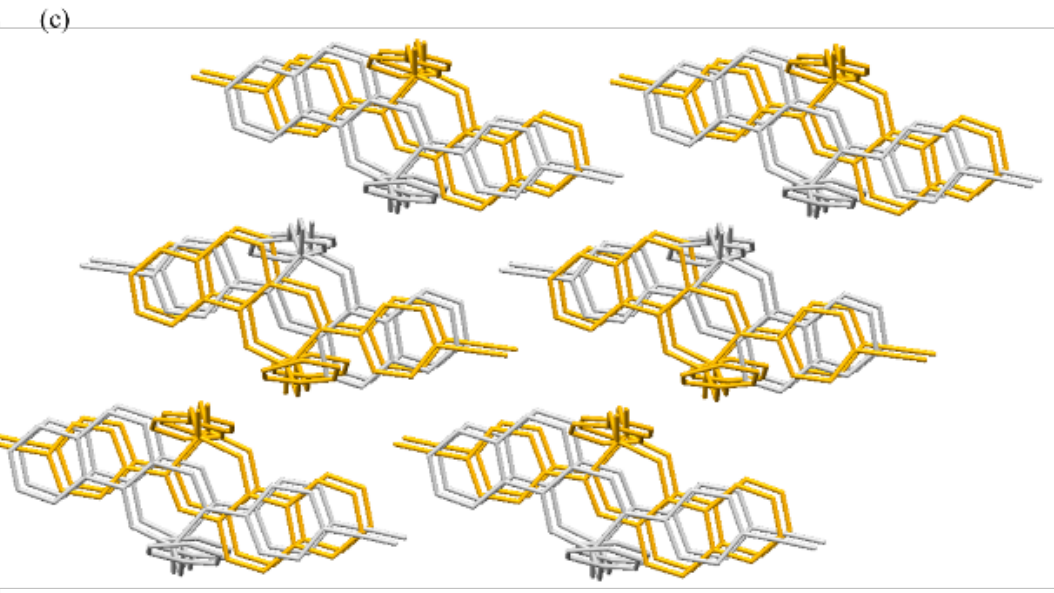

Figure S2. (a) ORTEP drawing of the dimer structure of $\mathbf{8 c}$ via hydrogen-bonding (thermal ellipsoids drawn at $30 \%$ probability). Single crystals suitable for X-ray diffraction were obtained by slow evaporation of $\mathrm{CHCl}_{3}$ solution at room temperature; (b) Intermolecular $\pi$ contacts; (c) The molecular packing diagram viewed along the $a$-axis. All hydrogen atoms not involved in $\mathrm{H}$-bonding interactions are omitted for clarity; the distances of contacts are in $\AA$. 
Table S1. Selected bond lengths, bond angles, and dihedral angles of PN-heterocycles $\mathbf{8 c}$ and $\mathbf{8 d}$. There are no significant differences of the selected bond angles and lengths among the two structures. The dihedral angle between the tricyclic cores and their intersections with the pendant phenyl plane exhibit the value of $7^{\circ}$ for $\mathbf{8 c}$ and $40^{\circ}$ in $\mathbf{8 d}$, respectively, which is likely attributable to the packing effects in the solid state.

\begin{tabular}{|c|c|c|}
\hline Bond lengths & Bond angles & gles $A / B$ \\
\hline Compound & $8 c$ & 8d \\
\hline \multicolumn{3}{|l|}{ Bond length $(\AA)$} \\
\hline$a$ & $1.632 / 1.635$ & 1.648 \\
\hline$b$ & $1.771 / 1.760$ & 1.762 \\
\hline$c$ & $1.353 / 1.351$ & 1.347 \\
\hline \multicolumn{3}{|l|}{ Bond angle $\left(^{\circ}\right)$} \\
\hline$\alpha$ & $128.2 / 128.9$ & 127.4 \\
\hline$\beta$ & $128.1 / 128.2$ & 127.8 \\
\hline Deviation of $P$ atom $(\AA)^{[\mathrm{a}]}$ & $0.05 / 0.11$ & 0.25 \\
\hline Dihedral angle $\mathrm{A} / \mathbf{B} \boldsymbol{\theta}\left({ }^{\mathrm{o}}\right)^{[\mathrm{b}]}$ & 7.1/7.6 & 40.2 \\
\hline
\end{tabular}

${ }^{a}$ The average distance of the $\mathrm{P}$ atom from the mean plane $\mathbf{A}$ defined by the other 13 non- $\mathrm{H}$ atoms in the tricyclic core. ${ }^{b}$ Dihedral angles between the mean plane A defined by the other 13 non- $\mathrm{H}$ atoms and the pendant phenyl plane B. 


\section{Photophysical Properties}

UV/Vis data were obtained on an HP $8453 \mathrm{UV} / \mathrm{Vis}$ spectrometer. Fluorescence data were acquired with a Horiba Jobin-Yvon FluoroMax-4 fluorescence spectrophotometer. Dilute solutions in degassed spectral grade $\mathrm{CHCl}_{3}$ in a $1 \mathrm{~cm}$ quartz cell were used for measurements. Quantum yields were calculated by comparison with freshly prepared quinine sulfate in $0.1 \mathrm{M}$ $\mathrm{H}_{2} \mathrm{SO}_{4}$ with excitation at $360 \mathrm{~nm}$ using an excitation slit width of $2.5 \mathrm{~nm}$ for all samples, and emission integrated across the range 380-650 $\mathrm{nm}$. To minimize the re-absorption effects, the absorbance in the $10 \mathrm{~mm}$ fluorescence cuvette was about 0.05-0.07 at $360 \mathrm{~nm}$. Fluorescence lifetimes were measured using time correlated single photon counting (TCSPC). Dilute solutions were prepared and placed in a $1 \mathrm{~cm}$ optical path quartz cuvette. A pulsed nanoLED was used to excite the samples at $344 \mathrm{~nm}$ at a $1 \mathrm{MHz}$ repetition rate. The emission was detected via a longpass filter. The decay profiles of were fitted reasonably well using the bi-exponential (for 8a-c) and mono-exponential functions (for 8d-f).

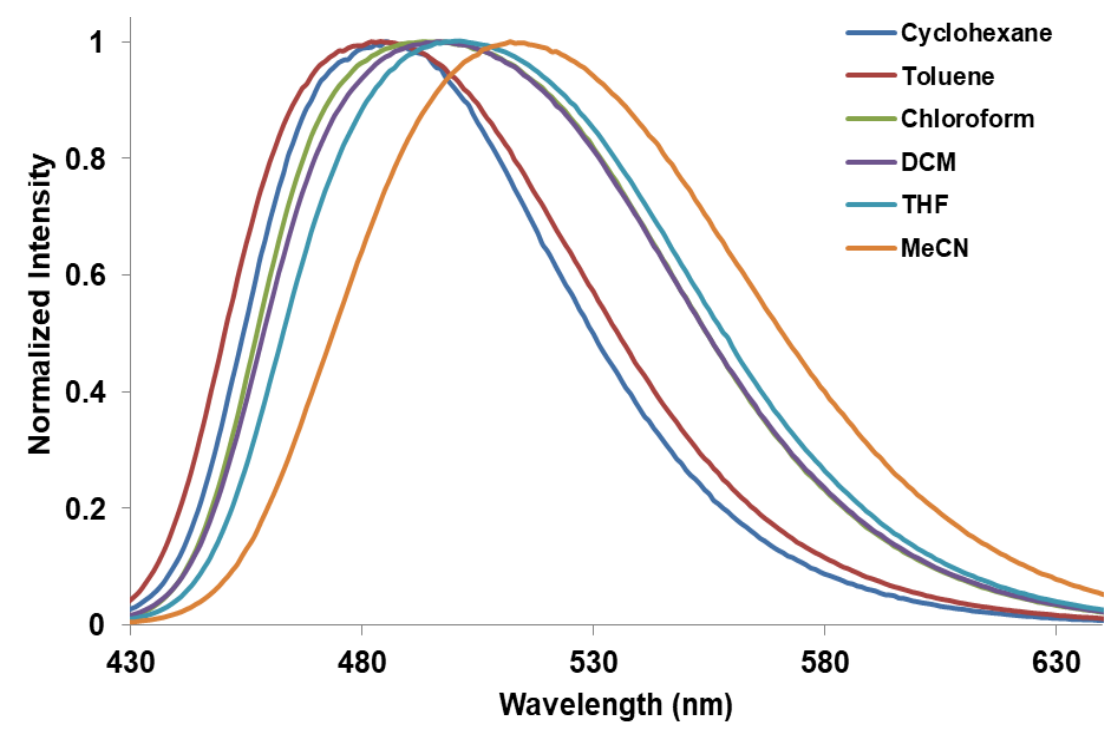

Figure S3. Fluorescence spectra of $\mathbf{8 a}$ in various solvents. 


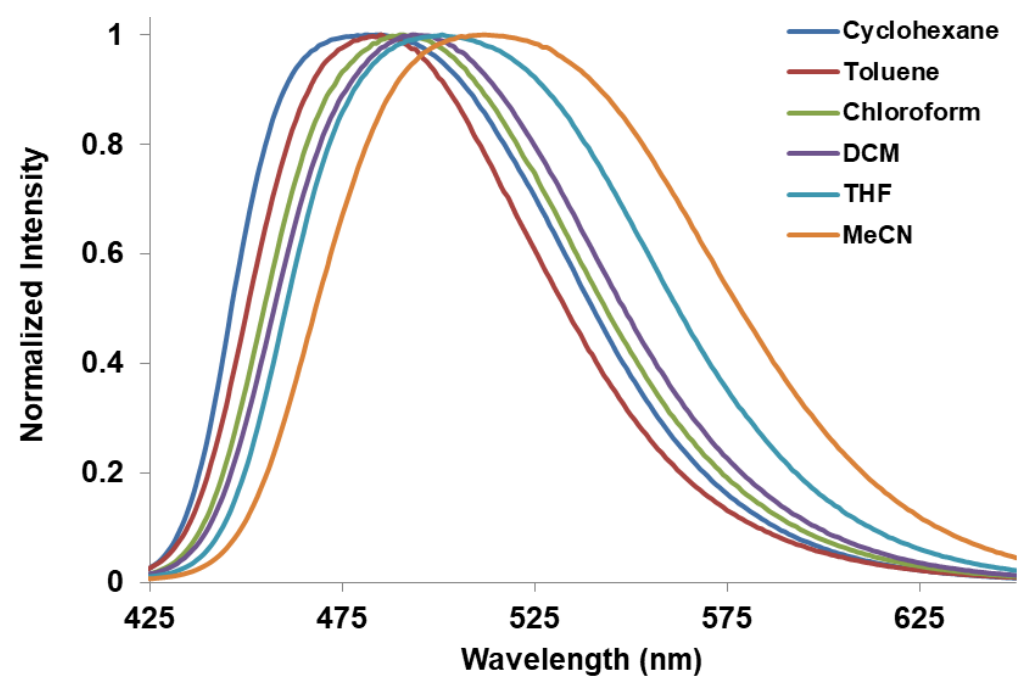

Figure S4. Fluorescence spectra of $\mathbf{8 b}$ in various solvents.

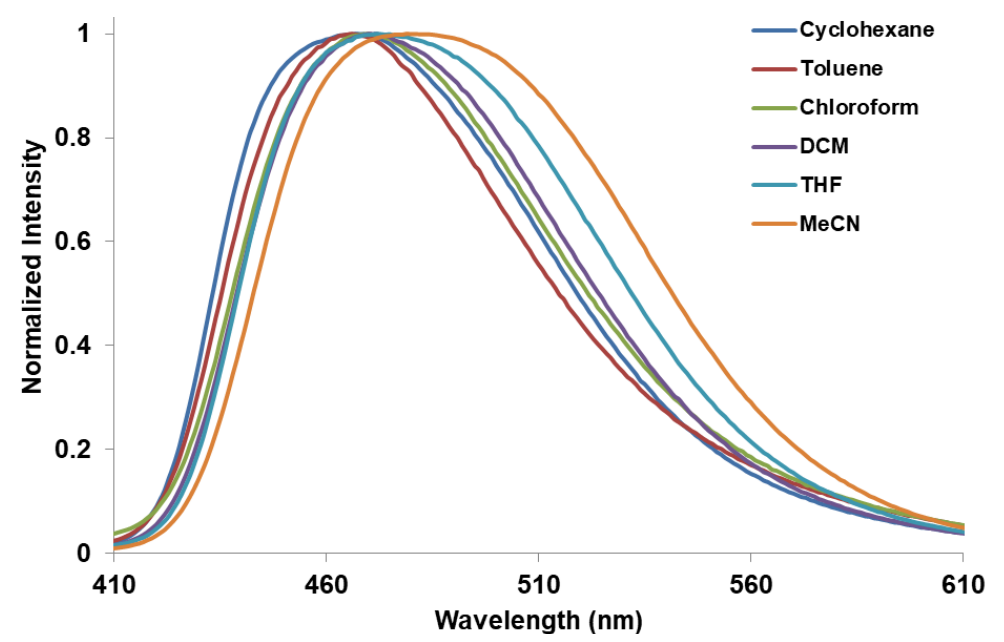

Figure S5. Fluorescence spectra of $8 \mathrm{c}$ in various solvents.

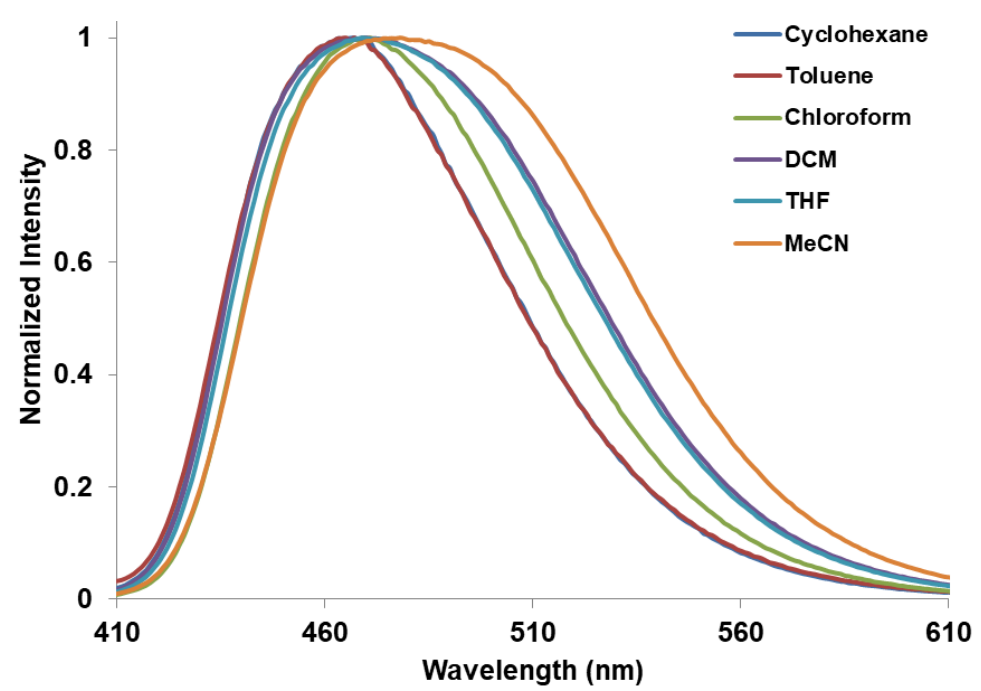

Figure S6. Fluorescence spectra of $\mathbf{8 d}$ in various solvents. 


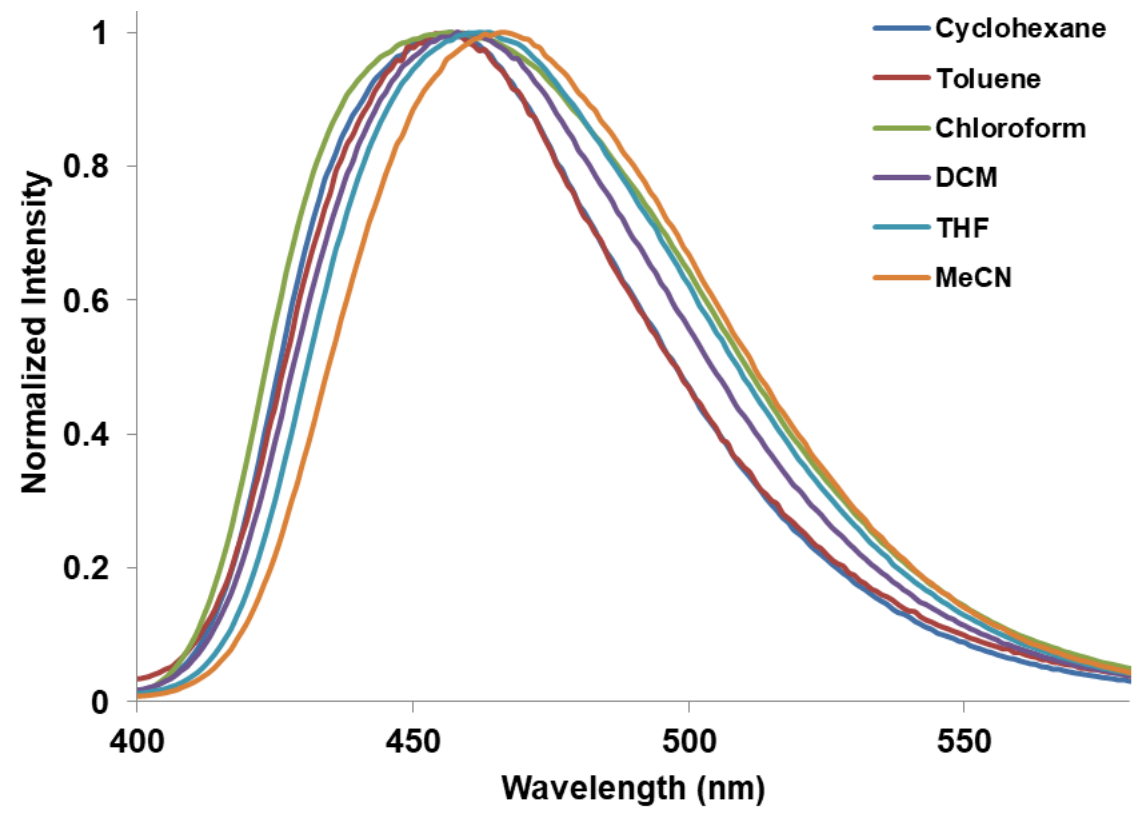

Figure S7. Fluorescence spectra of $\mathbf{8 e}$ in various solvents.

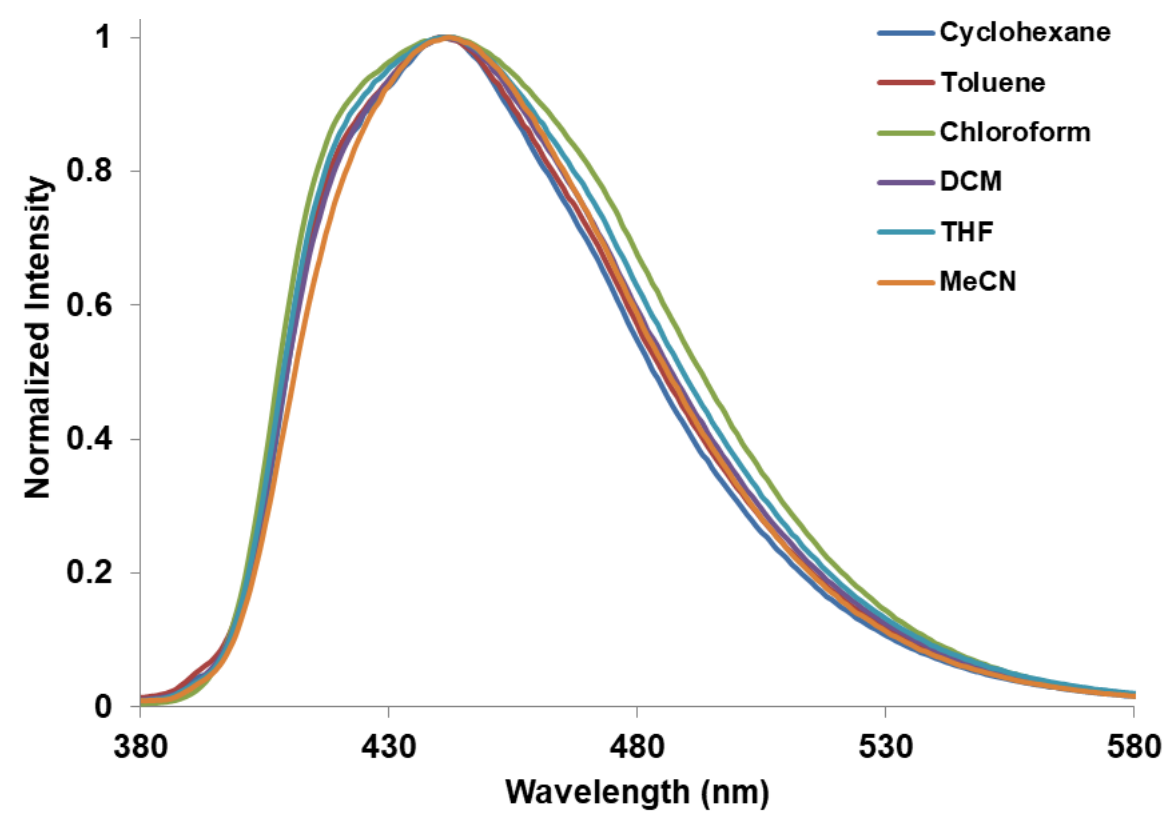

Figure S8. Fluorescence spectra of $\mathbf{8 f}$ in various solvents. 


\section{Theoretical calculations}

This work benefited from access to the University of Oregon high performance computer, Talapas. The initial structures of 7, 8c and $8 \mathbf{d}$ were generated from the above X-ray crystallography data. Other similar structures are adjusted according to the structures in the single crystal.

\subsection{TD-DFT calculations}

These initial structures were optimized using the functional PBE0 ${ }^{7}$ and $6-311 \mathrm{G}(\mathrm{d})$ basis set as implemented in Gaussian 09. ${ }^{8}$ In addition, all the optimized structures were confirmed by frequency analysis and the number of imaginary frequencies was zero. TD-DFT vertical excitation calculations and geometry optimization of the first excited state $\left(\mathrm{S}_{1}\right)$ were performed at the same level of theory. The PCM solvation model $^{9}$ was used to account for the solvent effects of the chloroform. 


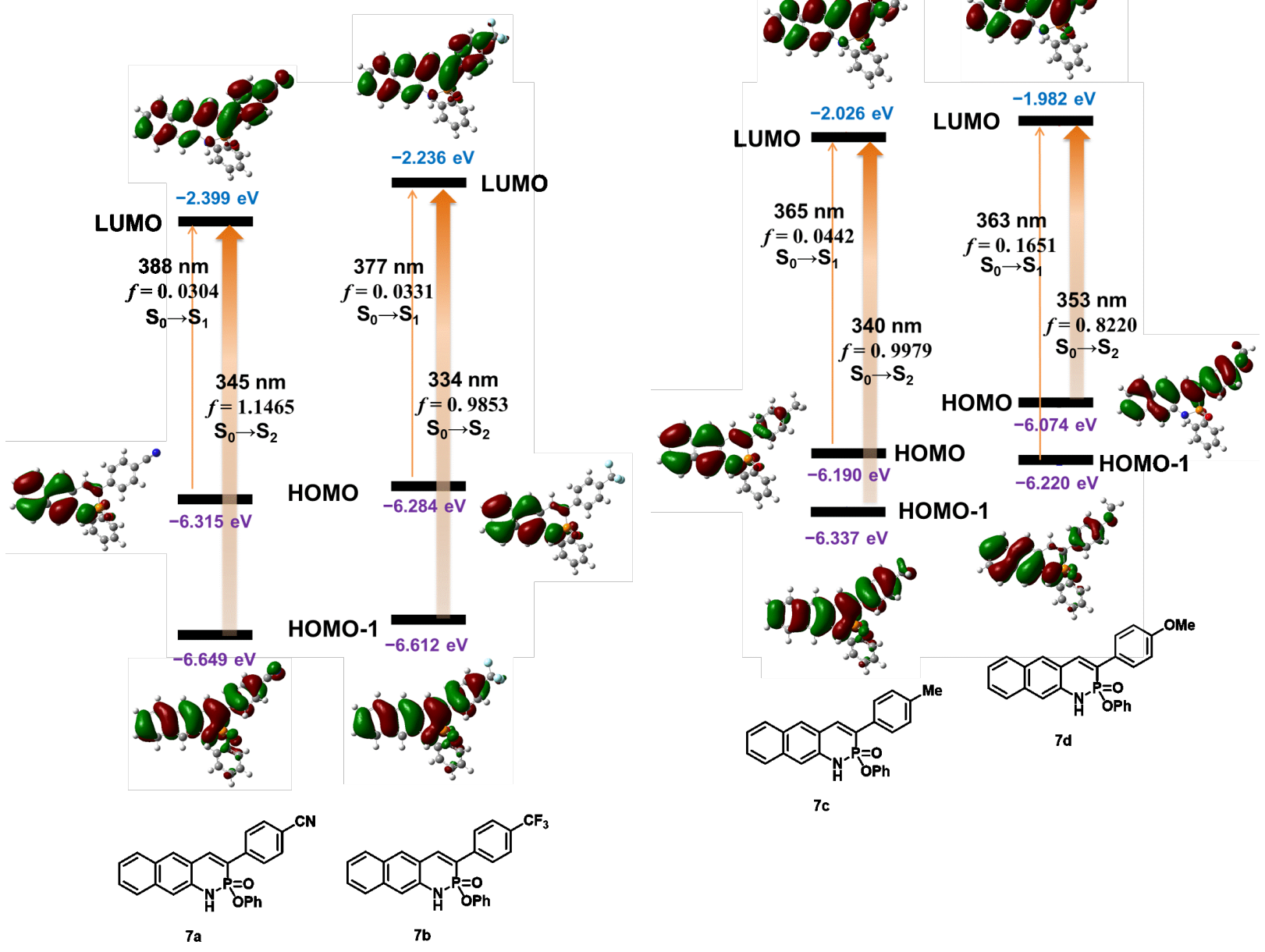

Figure S9. Kohn-Sham molecular orbitals, excitation energies, and oscillator strengths for PNheterocycles 7a-d, calculated at the TD-PBE0/6-311G(d) level of theory. For 7a-c, the $\mathrm{S}_{0} \rightarrow \mathrm{S}_{1}$ electronic transitions are dominated by the transitions from HOMO $\rightarrow$ LUMO with very small oscillator strengths $(f$ $<0.1$ ), while the transitions from HOMO-1 $\rightarrow$ LUMO contribute the $\mathrm{S}_{0} \rightarrow \mathrm{S}_{2}$ electronic transitions with large oscillator strengths $(f \approx 1)$. For $7 \mathbf{d}$, the $\mathrm{S}_{0} \rightarrow \mathrm{S}_{1}$ electronic transitions mainly correspond to the transitions from HOMO- $1 \rightarrow$ LUMO, while the $\mathrm{S}_{0} \rightarrow \mathrm{S}_{2}$ electronic transitions is predominated by the transitions from $\mathrm{HOMO} \rightarrow$ LUMO. 
Table S2. The $\mathrm{S}_{0} / \mathrm{S}_{1}$ geometries of 8 .

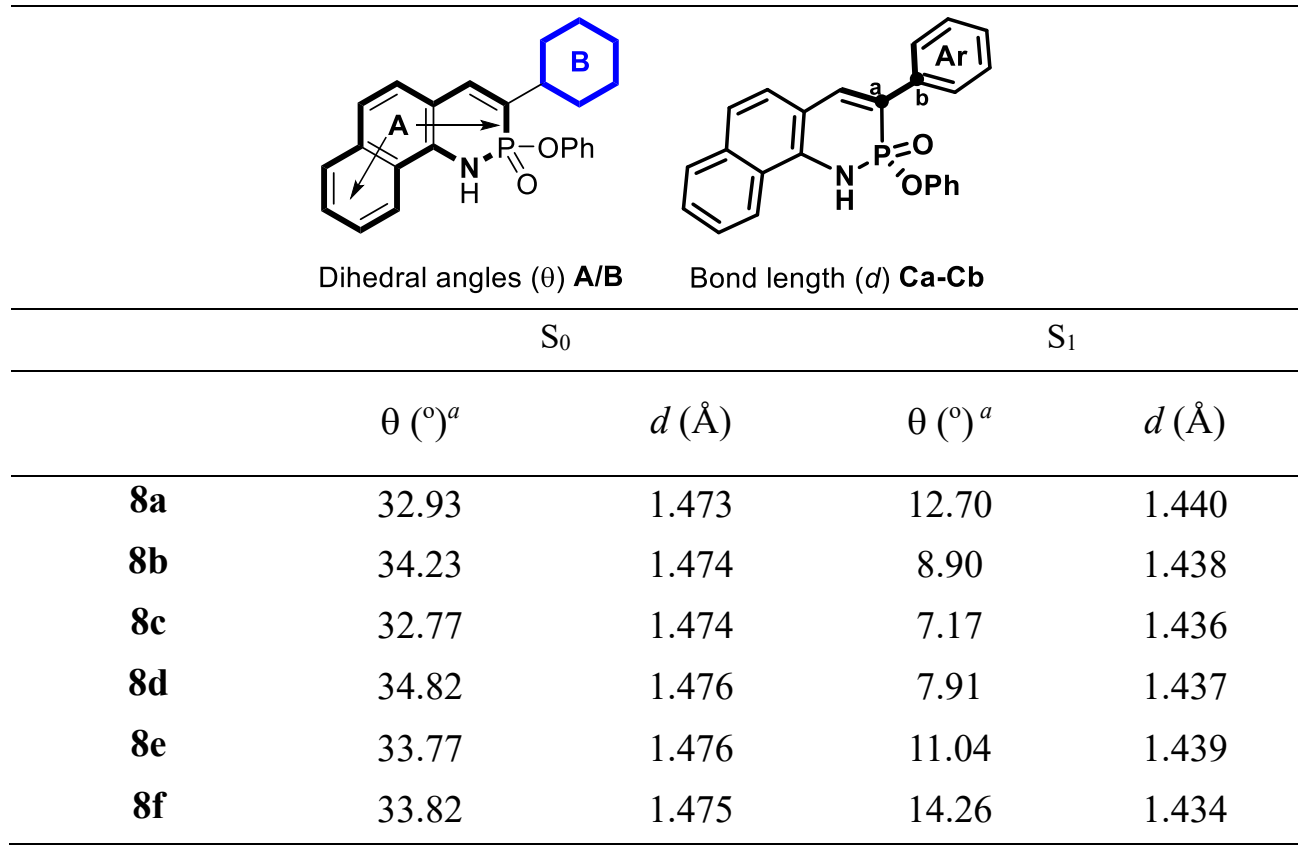

${ }^{a}$ Dihedral angles between the mean plane A defined by the other 13 non- $\mathrm{H}$ atoms and the pendant phenyl plane $\mathbf{B}$.

Table S3. The frontier molecular orbital energies in the $\mathrm{S}_{1}$ states and TD-DFT predicted emission wavelengths of 8 .

\begin{tabular}{cccccc}
\hline & HOMO $(\mathrm{eV})$ & LUMO $(\mathrm{eV})$ & $\Delta E_{\mathrm{H}-\mathrm{L}}(\mathrm{eV})$ & $\lambda_{\text {em,cal }}(\mathrm{nm})^{a}$ & $\lambda_{\text {em,exp }}(\mathrm{nm})$ \\
\hline $\mathbf{8 a}$ & -6.144 & -2.948 & 3.196 & $485(0.6025)$ & 493 \\
$\mathbf{8 b}$ & -6.124 & -2.86 & 3.264 & $477(0.6002)$ & 490 \\
$\mathbf{8 c}$ & -6.023 & -2.721 & 3.302 & $468(0.7179)$ & 469 \\
$\mathbf{8 d}$ & -6.042 & -2.68 & 3.362 & $461(0.6329)$ & 470 \\
$\mathbf{8 e}$ & -5.98 & -2.569 & 3.411 & $452(0.5917)$ & 457 \\
$\mathbf{8 f}$ & -5.592 & -2.305 & 3.287 & $461(0.9252)$ & 441 \\
\hline
\end{tabular}

${ }^{a}$ The calculated oscillator strength $(f)$ given in parentheses. 
Table S4. Cartesian coordinates for compound 8a $\left(\mathrm{S}_{0}\right)$.

\begin{tabular}{|c|c|c|c|}
\hline & $X$ & $\mathrm{Y}$ & Z \\
\hline $\mathrm{C}$ & -3.35271900 & -1.20420200 & 2.53300500 \\
\hline $\mathrm{C}$ & -2.00606500 & -1.14125900 & 2.35750400 \\
\hline $\mathrm{C}$ & -4.22602400 & -1.08607300 & 1.41893800 \\
\hline $\mathrm{C}$ & -3.69077000 & -0.89475000 & 0.11278200 \\
\hline $\mathrm{C}$ & -2.26894600 & -0.84593400 & -0.04256000 \\
\hline $\mathrm{C}$ & -1.43143400 & -0.96170300 & 1.06865300 \\
\hline $\mathrm{N}$ & -1.71933000 & -0.70538700 & -1.29922200 \\
\hline $\mathrm{P}$ & -0.11889700 & -0.43998600 & -1.72505300 \\
\hline $\mathrm{C}$ & 0.72457600 & -0.77490500 & -0.19769700 \\
\hline $\mathrm{C}$ & -0.00503900 & -0.95929700 & 0.93481100 \\
\hline $\mathrm{O}$ & 0.28286000 & -1.14480500 & -2.96141600 \\
\hline $\mathrm{C}$ & 2.19697100 & -0.80638500 & -0.18933100 \\
\hline $\mathrm{C}$ & 2.90576700 & -0.30244100 & 0.90712000 \\
\hline $\mathrm{C}$ & 4.29465400 & -0.35462200 & 0.94599400 \\
\hline $\mathrm{C}$ & 5.01025000 & -0.90607100 & -0.13436900 \\
\hline $\mathrm{C}$ & 4.30590600 & -1.38831500 & -1.23642400 \\
\hline $\mathrm{C}$ & 2.92235600 & -1.33665000 & -1.26534100 \\
\hline $\mathrm{C}$ & -5.62753400 & -1.14792400 & 1.58124800 \\
\hline $\mathrm{C}$ & -6.47254400 & -1.02166000 & 0.50731500 \\
\hline $\mathrm{C}$ & -5.94612600 & -0.81825400 & -0.78126800 \\
\hline $\mathrm{C}$ & -4.58803400 & -0.75466000 & -0.97185700 \\
\hline $\mathrm{O}$ & 0.03899600 & 1.15615200 & -2.00919200 \\
\hline $\mathrm{C}$ & -0.27586800 & 2.13487200 & -1.07858700 \\
\hline $\mathrm{C}$ & -1.58530700 & 2.58133300 & -0.95811100 \\
\hline $\mathrm{C}$ & -1.87333300 & 3.60036900 & -0.05744800 \\
\hline $\mathrm{C}$ & -0.86157200 & 4.16953800 & 0.70809200 \\
\hline $\mathrm{C}$ & 0.44713200 & 3.71957800 & 0.56861400 \\
\hline $\mathrm{C}$ & 0.74589600 & 2.69858200 & -0.32581700 \\
\hline $\mathrm{C}$ & 6.43288500 & -0.96755300 & -0.11363100 \\
\hline $\mathrm{N}$ & 7.58579900 & -1.02406300 & -0.10777500 \\
\hline $\mathrm{H}$ & -3.77670500 & -1.34565800 & 3.52182700 \\
\hline $\mathrm{H}$ & -1.33787800 & -1.23336800 & 3.20848900 \\
\hline $\mathrm{H}$ & -2.34428500 & -0.80180400 & -2.08693400 \\
\hline $\mathrm{H}$ & 0.54400900 & -1.16380900 & 1.85277400 \\
\hline $\mathrm{H}$ & 2.37779700 & 0.15847600 & 1.73386400 \\
\hline $\mathrm{H}$ & 4.85006700 & -1.81113200 & -2.07275300 \\
\hline $\mathrm{H}$ & 2.39189700 & -1.72135800 & -2.12874700 \\
\hline $\mathrm{H}$ & -6.02784800 & -1.29689400 & 2.57972800 \\
\hline $\mathrm{H}$ & -7.54716800 & -1.07167100 & 0.64978200 \\
\hline $\mathrm{H}$ & -6.61475800 & -0.70588500 & -1.62817700 \\
\hline $\mathrm{H}$ & -4.22565600 & -0.57795000 & -1.97980500 \\
\hline $\mathrm{H}$ & -2.36366000 & 2.14011600 & -1.57070400 \\
\hline $\mathrm{H}$ & -2.89513200 & 3.95265200 & 0.04006500 \\
\hline $\mathrm{H}$ & -1.09117700 & 4.96744100 & 1.40660700 \\
\hline $\mathrm{H}$ & 1.24364100 & 4.16664600 & 1.15473700 \\
\hline $\mathrm{H}$ & 1.76299800 & 2.34545800 & -0.45811700 \\
\hline
\end{tabular}




$$
\begin{array}{llll}
\mathrm{C} & 4.98087700 & 0.17131800 & 2.08058100 \\
\mathrm{~N} & 5.52306600 & 0.59947600 & 3.00481400
\end{array}
$$

\begin{tabular}{|c|c|c|c|}
\hline & $\mathrm{X}$ & $\mathrm{Y}$ & $\mathrm{Z}$ \\
\hline $\mathrm{C}$ & -3.39447600 & -2.12734200 & 1.75355100 \\
\hline $\mathrm{C}$ & -2.02528300 & -2.03930100 & 1.59915400 \\
\hline $\mathrm{C}$ & -4.27675300 & -1.51497200 & 0.83190700 \\
\hline $\mathrm{C}$ & -3.73039200 & -0.79758600 & -0.27517300 \\
\hline $\mathrm{C}$ & -2.31187500 & -0.74729100 & -0.42997500 \\
\hline $\mathrm{C}$ & -1.43492500 & -1.35307100 & 0.52811900 \\
\hline $\mathrm{N}$ & -1.75001000 & -0.15357300 & -1.51464900 \\
\hline $\mathrm{P}$ & -0.12124800 & 0.26906500 & -1.77801700 \\
\hline $\mathrm{C}$ & 0.75173900 & -0.66481500 & -0.54281800 \\
\hline $\mathrm{C}$ & -0.01344400 & -1.32565400 & 0.41045600 \\
\hline $\mathrm{O}$ & 0.20981400 & 0.15284300 & -3.21226200 \\
\hline $\mathrm{C}$ & 2.19062500 & -0.60187600 & -0.52644900 \\
\hline $\mathrm{C}$ & 2.91774600 & -0.99630100 & 0.62227200 \\
\hline $\mathrm{C}$ & 4.29830700 & -0.94713600 & 0.66366100 \\
\hline $\mathrm{C}$ & 5.04517300 & -0.49710900 & -0.45929100 \\
\hline $\mathrm{C}$ & 4.32601600 & -0.09651400 & -1.60345100 \\
\hline $\mathrm{C}$ & 2.95469800 & -0.14534700 & -1.64003100 \\
\hline $\mathrm{C}$ & -5.66833100 & -1.59125500 & 0.99522700 \\
\hline $\mathrm{C}$ & -6.52744800 & -0.97478600 & 0.09997700 \\
\hline $\mathrm{C}$ & -5.99720500 & -0.25894400 & -0.97570300 \\
\hline $\mathrm{C}$ & -4.62653900 & -0.16853900 & -1.16052300 \\
\hline $\mathrm{O}$ & -0.02208100 & 1.83396500 & -1.35360300 \\
\hline $\mathrm{C}$ & -0.26250000 & 2.31781200 & -0.07481200 \\
\hline $\mathrm{C}$ & -1.54839700 & 2.70981100 & 0.27179300 \\
\hline $\mathrm{C}$ & -1.77463400 & 3.24417400 & 1.53535100 \\
\hline $\mathrm{C}$ & -0.72427600 & 3.38403800 & 2.43550200 \\
\hline $\mathrm{C}$ & 0.55979100 & 2.99253700 & 2.06913100 \\
\hline $\mathrm{C}$ & 0.79810700 & 2.45550600 & 0.81004000 \\
\hline $\mathrm{C}$ & 6.45416900 & -0.44525000 & -0.43628400 \\
\hline $\mathrm{N}$ & 7.61298300 & -0.39646400 & -0.42898000 \\
\hline $\mathrm{H}$ & -3.80704800 & -2.67336800 & 2.59554300 \\
\hline $\mathrm{H}$ & -1.37681100 & -2.52309600 & 2.32220300 \\
\hline $\mathrm{H}$ & -2.36032300 & 0.12362600 & -2.27251800 \\
\hline $\mathrm{H}$ & 0.50901900 & -1.90364800 & 1.16590000 \\
\hline $\mathrm{H}$ & 2.39902300 & -1.31616100 & 1.51747200 \\
\hline $\mathrm{H}$ & 4.87369400 & 0.24689100 & -2.47399100 \\
\hline $\mathrm{H}$ & 2.45137700 & 0.14512500 & -2.55516000 \\
\hline $\mathrm{H}$ & -6.06478900 & -2.14278700 & 1.84209900 \\
\hline $\mathrm{H}$ & -7.60086100 & -1.04170800 & 0.23633500 \\
\hline $\mathrm{H}$ & -6.66013400 & 0.23804400 & -1.67568500 \\
\hline $\mathrm{H}$ & -4.26880200 & 0.41942600 & -1.99977100 \\
\hline $\mathrm{H}$ & -2.35364100 & 2.60705000 & -0.44772700 \\
\hline $\mathrm{H}$ & -2.77643000 & 3.55649000 & 1.81190700 \\
\hline
\end{tabular}

Table S5. Cartesian coordinates for compound 8a $\left(\mathrm{S}_{1}\right)$. 


$\begin{array}{lrrr}\mathrm{H} & -0.90412400 & 3.80414100 & 3.41959300 \\ \mathrm{H} & 1.38476600 & 3.10765800 & 2.76456100 \\ \mathrm{H} & 1.79360800 & 2.15239500 & 0.50501000 \\ \mathrm{C} & 4.95960000 & -1.34377300 & 1.86206800 \\ \mathrm{~N} & 5.48579500 & -1.66890600 & 2.83780300\end{array}$

Table S6. Cartesian coordinates for compound $\mathbf{8 b}\left(\mathrm{S}_{0}\right)$.

\begin{tabular}{|c|c|c|c|}
\hline & $\mathrm{X}$ & $\mathrm{Y}$ & $\mathrm{Z}$ \\
\hline $\mathrm{C}$ & 4.73875000 & -1.43517200 & -2.28175300 \\
\hline $\mathrm{C}$ & 3.38197200 & -1.38595600 & -2.20740100 \\
\hline $\mathrm{C}$ & 5.52825800 & -1.16648200 & -1.13207700 \\
\hline $\mathrm{C}$ & 4.89818700 & -0.84098300 & 0.10352900 \\
\hline $\mathrm{C}$ & 3.46806700 & -0.81021400 & 0.15356700 \\
\hline $\mathrm{C}$ & 2.71431400 & -1.07249600 & -0.99159800 \\
\hline $\mathrm{N}$ & 2.82636300 & -0.53873000 & 1.34344500 \\
\hline $\mathrm{P}$ & 1.19429900 & -0.26707600 & 1.62376900 \\
\hline $\mathrm{C}$ & 0.46802500 & -0.78507500 & 0.08743500 \\
\hline $\mathrm{C}$ & 1.28127800 & -1.08275700 & -0.96023500 \\
\hline $\mathrm{O}$ & 0.72306200 & -0.83867900 & 2.90352000 \\
\hline $\mathrm{C}$ & -1.00025200 & -0.83686400 & -0.02966900 \\
\hline $\mathrm{C}$ & -1.62391200 & -0.44697000 & -1.22465400 \\
\hline $\mathrm{C}$ & -2.99823200 & -0.51720400 & -1.36605500 \\
\hline $\mathrm{C}$ & -3.75856500 & -0.97670300 & -0.29450200 \\
\hline $\mathrm{C}$ & -3.17249900 & -1.35437600 & 0.90940100 \\
\hline $\mathrm{C}$ & -1.79530200 & -1.28061700 & 1.03743300 \\
\hline $\mathrm{C}$ & 6.93874800 & -1.20886600 & -1.19054100 \\
\hline $\mathrm{C}$ & 7.70360500 & -0.93801400 & -0.08387800 \\
\hline $\mathrm{C}$ & 7.08360100 & -0.60394000 & 1.13390600 \\
\hline $\mathrm{C}$ & 5.71441900 & -0.55539600 & 1.22335200 \\
\hline $\mathrm{O}$ & 0.99085600 & 1.34769100 & 1.71720600 \\
\hline $\mathrm{C}$ & 1.31463000 & 2.22192600 & 0.69138100 \\
\hline $\mathrm{C}$ & 2.61937600 & 2.67679300 & 0.55161500 \\
\hline $\mathrm{C}$ & 2.91370800 & 3.59419300 & -0.45074100 \\
\hline $\mathrm{C}$ & 1.91267800 & 4.05532500 & -1.29860400 \\
\hline $\mathrm{C}$ & 0.60843900 & 3.59844800 & -1.14026800 \\
\hline $\mathrm{C}$ & 0.30371900 & 2.67802300 & -0.14450700 \\
\hline $\mathrm{H}$ & 5.23436700 & -1.68014500 & -3.21556100 \\
\hline $\mathrm{H}$ & 2.77696700 & -1.59276600 & -3.08513700 \\
\hline $\mathrm{H}$ & 3.39266400 & -0.53046800 & 2.17965800 \\
\hline $\mathrm{H}$ & 0.80212900 & -1.39806300 & -1.88588600 \\
\hline $\mathrm{H}$ & -1.02764600 & -0.06191800 & -2.04459000 \\
\hline $\mathrm{H}$ & -3.78334000 & -1.72121100 & 1.72629800 \\
\hline $\mathrm{H}$ & -1.32611200 & -1.58310200 & 1.96642000 \\
\hline $\mathrm{H}$ & 7.41089600 & -1.46035000 & -2.13569300 \\
\hline $\mathrm{H}$ & 8.78636300 & -0.97521000 & -0.14619100 \\
\hline $\mathrm{H}$ & 7.68849900 & -0.37899400 & 2.00599700 \\
\hline $\mathrm{H}$ & 5.27910500 & -0.27678600 & 2.17798600 \\
\hline $\mathrm{H}$ & 3.38946500 & 2.32001400 & 1.22640100 \\
\hline $\mathrm{H}$ & 3.93202400 & 3.95204200 & -0.56362900 \\
\hline
\end{tabular}




$\begin{array}{lrrr}\mathrm{H} & 2.14713300 & 4.77447100 & -2.07657400 \\ \mathrm{H} & -0.17993300 & 3.96130500 & -1.79202800 \\ \mathrm{H} & -0.70952700 & 2.31856400 & -0.00040700 \\ \mathrm{H} & -3.47689300 & -0.22392500 & -2.29326100 \\ \mathrm{~S} & -5.51126100 & -1.06128500 & -0.46401300 \\ \mathrm{O} & -5.86831300 & -1.08042600 & -1.87223300 \\ \mathrm{O} & -6.05471700 & -2.02290700 & 0.47894400 \\ \mathrm{C} & -6.06094100 & 0.61549800 & 0.16137000 \\ \mathrm{~F} & -7.37311100 & 0.70025800 & 0.03456800 \\ \mathrm{~F} & -5.72797100 & 0.75218700 & 1.43431100 \\ \mathrm{~F} & -5.48497600 & 1.57157500 & -0.55096500\end{array}$

Table S7. Cartesian coordinates for compound $\mathbf{8 b}\left(\mathrm{S}_{1}\right)$.

\begin{tabular}{|c|c|c|c|}
\hline & $\mathrm{X}$ & $\mathrm{Y}$ & Z \\
\hline $\mathrm{C}$ & 4.73844200 & -1.02285500 & -2.42829800 \\
\hline $\mathrm{C}$ & 3.36401800 & -1.07703900 & -2.32342900 \\
\hline $\mathrm{C}$ & 5.55623200 & -0.85437600 & -1.28427200 \\
\hline $\mathrm{C}$ & 4.93497000 & -0.73861300 & -0.00284400 \\
\hline $\mathrm{C}$ & 3.51337700 & -0.81943100 & 0.08209700 \\
\hline $\mathrm{C}$ & 2.70057100 & -0.97416000 & -1.09064600 \\
\hline $\mathrm{N}$ & 2.88168400 & -0.79074000 & 1.28519200 \\
\hline $\mathrm{P}$ & 1.22420400 & -0.57674800 & 1.61253900 \\
\hline $\mathrm{C}$ & 0.44510400 & -0.94839600 & 0.06136900 \\
\hline $\mathrm{C}$ & 1.28084400 & -1.06846400 & -1.04902200 \\
\hline $\mathrm{O}$ & 0.86234700 & -1.27827200 & 2.86095900 \\
\hline $\mathrm{C}$ & -0.99090700 & -0.97457500 & -0.00597500 \\
\hline $\mathrm{C}$ & -1.66767200 & -0.93470200 & -1.25989600 \\
\hline $\mathrm{C}$ & -3.03818400 & -0.95575900 & -1.34042300 \\
\hline $\mathrm{C}$ & -3.80309900 & -1.03354400 & -0.16594400 \\
\hline $\mathrm{C}$ & -3.17273700 & -1.06855900 & 1.09006400 \\
\hline $\mathrm{C}$ & -1.80064600 & -1.04532000 & 1.16207100 \\
\hline $\mathrm{C}$ & 6.95313700 & -0.79117600 & -1.39063100 \\
\hline $\mathrm{C}$ & 7.74877000 & -0.61487100 & -0.26928100 \\
\hline $\mathrm{C}$ & 7.14582500 & -0.48997200 & 0.98447200 \\
\hline $\mathrm{C}$ & 5.76768700 & -0.54772800 & 1.11788800 \\
\hline $\mathrm{O}$ & 1.05366300 & 1.02316900 & 1.86056100 \\
\hline $\mathrm{C}$ & 1.27965200 & 1.99634700 & 0.89921400 \\
\hline $\mathrm{C}$ & 2.54384500 & 2.55972700 & 0.78638300 \\
\hline $\mathrm{C}$ & 2.75235800 & 3.57396400 & -0.14166600 \\
\hline $\mathrm{C}$ & 1.70608900 & 4.01656200 & -0.94313500 \\
\hline $\mathrm{C}$ & 0.44366600 & 3.44538400 & -0.81305000 \\
\hline $\mathrm{C}$ & 0.22315600 & 2.43053900 & 0.10969400 \\
\hline $\mathrm{H}$ & 5.20777300 & -1.11149700 & -3.40266800 \\
\hline $\mathrm{H}$ & 2.76684200 & -1.21221700 & -3.21937200 \\
\hline $\mathrm{H}$ & 3.44798900 & -0.84182200 & 2.12163100 \\
\hline $\mathrm{H}$ & 0.81803400 & -1.26903500 & -2.00923700 \\
\hline $\mathrm{H}$ & -1.10625700 & -0.85762400 & -2.18307000 \\
\hline $\mathrm{H}$ & -3.76669200 & -1.14335600 & 1.99392100 \\
\hline
\end{tabular}




$\begin{array}{lrrr}\mathrm{H} & -1.33318700 & -1.11599600 & 2.13776300 \\ \mathrm{H} & 7.40623300 & -0.88194900 & -2.37314600 \\ \mathrm{H} & 8.82777500 & -0.56966100 & -0.36457400 \\ \mathrm{H} & 7.75756600 & -0.34227200 & 1.86803400 \\ \mathrm{H} & 5.35329200 & -0.42599000 & 2.11353400 \\ \mathrm{H} & 3.34567000 & 2.21275300 & 1.42934500 \\ \mathrm{H} & 3.73698600 & 4.02111300 & -0.23238700 \\ \mathrm{H} & 1.87184300 & 4.81037600 & -1.66409600 \\ \mathrm{H} & -0.37844700 & 3.79278700 & -1.43034400 \\ \mathrm{H} & -0.75552600 & 1.97868000 & 0.22866200 \\ \mathrm{H} & -3.53153900 & -0.92296700 & -2.30552600 \\ \mathrm{~S} & -5.53234600 & -1.01773500 & -0.26719100 \\ \mathrm{O} & -5.96871900 & -1.41425300 & -1.59939100 \\ \mathrm{O} & -6.12238900 & -1.59609300 & 0.93211900 \\ \mathrm{C} & -5.97291700 & 0.79790800 & -0.16381300 \\ \mathrm{~F} & -7.28769900 & 0.93689900 & -0.24853400 \\ \mathrm{~F} & -5.55528300 & 1.30239700 & 0.98932300 \\ \mathrm{~F} & -5.40083500 & 1.46149300 & -1.16050000\end{array}$

Table S8. Cartesian coordinates for compound $8 c\left(\mathrm{~S}_{0}\right)$.

\begin{tabular}{|c|c|c|c|}
\hline & $\mathrm{X}$ & $\mathrm{Y}$ & $\mathrm{Z}$ \\
\hline $\mathrm{C}$ & 3.22839300 & -1.50801600 & -2.28489100 \\
\hline $\mathrm{C}$ & 1.87212300 & -1.43161200 & -2.21931200 \\
\hline $\mathrm{C}$ & 4.01564400 & -1.24997300 & -1.13149200 \\
\hline $\mathrm{C}$ & 3.38348800 & -0.90794400 & 0.09862200 \\
\hline $\mathrm{C}$ & 1.95411300 & -0.85146700 & 0.13955400 \\
\hline $\mathrm{C}$ & 1.20256600 & -1.10164900 & -1.00917800 \\
\hline $\mathrm{N}$ & 1.30932400 & -0.56705300 & 1.32512600 \\
\hline $\mathrm{P}$ & -0.31854500 & -0.26317700 & 1.59329700 \\
\hline $\mathrm{C}$ & -1.04683900 & -0.75996300 & 0.05073500 \\
\hline $\mathrm{C}$ & -0.23139700 & -1.08069800 & -0.98809400 \\
\hline $\mathrm{O}$ & -0.81281300 & -0.82908300 & 2.86726800 \\
\hline $\mathrm{C}$ & -2.51545000 & -0.76693000 & -0.07818800 \\
\hline $\mathrm{C}$ & -3.12096600 & -0.38675600 & -1.28509900 \\
\hline $\mathrm{C}$ & -4.49518200 & -0.41644300 & -1.43429500 \\
\hline $\mathrm{C}$ & -5.30257300 & -0.81644700 & -0.36410400 \\
\hline $\mathrm{C}$ & -4.71442200 & -1.17920900 & 0.85094000 \\
\hline $\mathrm{C}$ & -3.33758300 & -1.15164300 & 0.99047900 \\
\hline $\mathrm{C}$ & 5.42560300 & -1.31836000 & -1.18042500 \\
\hline $\mathrm{C}$ & 6.18816600 & -1.05634600 & -0.07010000 \\
\hline $\mathrm{C}$ & 5.56629300 & -0.70529500 & 1.14204800 \\
\hline $\mathrm{C}$ & 4.19766200 & -0.63184700 & 1.22228700 \\
\hline $\mathrm{O}$ & -0.48469800 & 1.35611800 & 1.69366700 \\
\hline $\mathrm{C}$ & -0.06631200 & 2.23123500 & 0.70419500 \\
\hline $\mathrm{C}$ & 1.24445400 & 2.69069100 & 0.70081000 \\
\hline $\mathrm{C}$ & 1.63880500 & 3.61019600 & -0.26413000 \\
\hline $\mathrm{C}$ & 0.72976400 & 4.06810200 & -1.21178300 \\
\hline $\mathrm{C}$ & -0.58189100 & 3.60574900 & -1.19102500 \\
\hline
\end{tabular}




$\begin{array}{lrrr}\mathrm{C} & -0.98621700 & 2.68360100 & -0.23280400 \\ \mathrm{C} & -6.72022900 & -0.84429600 & -0.51153200 \\ \mathrm{~N} & -7.86860300 & -0.86715600 & -0.63361700 \\ \mathrm{H} & 3.72490000 & -1.76603000 & -3.21475000 \\ \mathrm{H} & 1.26888100 & -1.62891300 & -3.10050600 \\ \mathrm{H} & 1.86963600 & -0.57065000 & 2.16525700 \\ \mathrm{H} & -0.70988700 & -1.38585300 & -1.91744200 \\ \mathrm{H} & -2.50876400 & -0.03941300 & -2.11055200 \\ \mathrm{H} & -4.94896400 & -0.11741800 & -2.37255800 \\ \mathrm{H} & -5.33807600 & -1.48671100 & 1.68281800 \\ \mathrm{H} & -2.88932500 & -1.44147500 & 1.93381800 \\ \mathrm{H} & 5.89919500 & -1.58243100 & -2.12145400 \\ \mathrm{H} & 7.27048200 & -1.11329100 & -0.12516100 \\ \mathrm{H} & 6.16951300 & -0.48675500 & 2.01697700 \\ \mathrm{H} & 3.76093900 & -0.34071200 & 2.17251400 \\ \mathrm{H} & 1.94009300 & 2.33562800 & 1.45322000 \\ \mathrm{H} & 2.66201600 & 3.97183200 & -0.27012500 \\ \mathrm{H} & 1.04124400 & 4.78851600 & -1.96107300 \\ \mathrm{H} & -1.29895100 & 3.96575700 & -1.92198100 \\ \mathrm{H} & -2.00778100 & 2.32065400 & -0.19580800\end{array}$

Table S9. Cartesian coordinates for compound $8 \mathbf{c}\left(\mathrm{S}_{1}\right)$.

\begin{tabular}{lrrr}
\hline & $\mathrm{X}$ & $\mathrm{Y}$ & $\mathrm{Z}$ \\
$\mathrm{C}$ & 3.33514800 & -1.34259400 & -2.26457200 \\
$\mathrm{C}$ & 1.96243200 & -1.34810100 & -2.20948500 \\
$\mathrm{C}$ & 4.11917700 & -1.06775800 & -1.11245100 \\
$\mathrm{C}$ & 3.45601000 & -0.79605500 & 0.12390200 \\
$\mathrm{C}$ & 2.03374400 & -0.83738200 & 0.16446100 \\
$\mathrm{C}$ & 1.25496000 & -1.09225200 & -1.01787800 \\
$\mathrm{~N}$ & 1.36298400 & -0.67871900 & 1.33844800 \\
$\mathrm{P}$ & -0.29629300 & -0.40177400 & 1.58589900 \\
$\mathrm{C}$ & -1.03348600 & -0.87292700 & 0.04278800 \\
$\mathrm{C}$ & -0.16058400 & -1.13381000 & -1.02219700 \\
$\mathrm{O}$ & -0.72198400 & -0.99490700 & 2.87094000 \\
$\mathrm{C}$ & -2.46289900 & -0.84506900 & -0.07987300 \\
$\mathrm{C}$ & -3.10060000 & -0.95791400 & -1.34909300 \\
$\mathrm{C}$ & -4.46806600 & -0.92614100 & -1.47563600 \\
$\mathrm{C}$ & -5.29202100 & -0.77946200 & -0.34351700 \\
$\mathrm{C}$ & -4.68470900 & -0.66514500 & 0.92333900 \\
$\mathrm{C}$ & -3.31676300 & -0.69929200 & 1.04927800 \\
$\mathrm{C}$ & 5.51799200 & -1.05039100 & -1.17064700 \\
$\mathrm{C}$ & 6.27926300 & -0.76831300 & -0.04549000 \\
$\mathrm{C}$ & 5.63679000 & -0.48720000 & 1.16289100 \\
$\mathrm{C}$ & 4.25508900 & -0.49706100 & 1.24910900 \\
$\mathrm{O}$ & -0.43499600 & 1.21680700 & 1.70987700 \\
$\mathrm{C}$ & -0.15417400 & 2.11091600 & 0.68875500 \\
$\mathrm{C}$ & 1.13068300 & 2.62345400 & 0.56627700 \\
$\mathrm{C}$ & 1.39369400 & 3.56219900 & -0.42520500 \\
$\mathrm{C}$ & 0.38059700 & 3.98129400 & -1.28014700
\end{tabular}




$\begin{array}{lrrr}\mathrm{C} & -0.90293300 & 3.46200600 & -1.14054500 \\ \mathrm{C} & -1.17756000 & 2.52227100 & -0.15478000 \\ \mathrm{C} & -6.69980900 & -0.74478700 & -0.47827100 \\ \mathrm{~N} & -7.85329100 & -0.71482200 & -0.59039400 \\ \mathrm{H} & 3.83852200 & -1.54950900 & -3.20360300 \\ \mathrm{H} & 1.39193700 & -1.56406100 & -3.10717300 \\ \mathrm{H} & 1.89879700 & -0.68444900 & 2.19610700 \\ \mathrm{H} & -0.59848600 & -1.40390300 & -1.97686400 \\ \mathrm{H} & -2.51012000 & -1.04835600 & -2.25277700 \\ \mathrm{H} & -4.91994600 & -1.00686500 & -2.45879700 \\ \mathrm{H} & -5.30397800 & -0.55875200 & 1.80747000 \\ \mathrm{H} & -2.88940000 & -0.64070000 & 2.04409700 \\ \mathrm{H} & 6.00368400 & -1.26144300 & -2.11879000 \\ \mathrm{H} & 7.36189800 & -0.76011800 & -0.10467300 \\ \mathrm{H} & 6.22154900 & -0.25299200 & 2.04623400 \\ \mathrm{H} & 3.80700400 & -0.24777800 & 2.20595900 \\ \mathrm{H} & 1.90736200 & 2.29521200 & 1.24868400 \\ \mathrm{H} & 2.39511300 & 3.96854800 & -0.52405100 \\ \mathrm{H} & 0.58894400 & 4.71626600 & -2.05075800 \\ \mathrm{H} & -1.69920200 & 3.79098100 & -1.80040200 \\ \mathrm{H} & -2.17316000 & 2.11123300 & -0.02813700\end{array}$

Table S10. Cartesian coordinates for compound $8 d\left(\mathrm{~S}_{0}\right)$.

\begin{tabular}{lrrr}
\hline & $\mathrm{X}$ & $\mathrm{Y}$ & $\mathrm{Z}$ \\
$\mathrm{C}$ & 3.96658600 & -1.62228000 & -2.28954600 \\
$\mathrm{C}$ & 2.61589300 & -1.49139300 & -2.19730000 \\
$\mathrm{C}$ & 4.78780800 & -1.37535800 & -1.15774900 \\
$\mathrm{C}$ & 4.19583200 & -0.98672300 & 0.07848900 \\
$\mathrm{C}$ & 2.77082100 & -0.87660800 & 0.14881700 \\
$\mathrm{C}$ & 1.98567900 & -1.11621400 & -0.97938800 \\
$\mathrm{~N}$ & 2.16315600 & -0.54964500 & 1.34365200 \\
$\mathrm{P}$ & 0.55589300 & -0.17100600 & 1.63707100 \\
$\mathrm{C}$ & -0.22434900 & -0.67273000 & 0.12268600 \\
$\mathrm{C}$ & 0.55367200 & -1.04105500 & -0.92816500 \\
$\mathrm{O}$ & 0.06314400 & -0.68190900 & 2.93448100 \\
$\mathrm{C}$ & -1.69610300 & -0.62978100 & 0.02847100 \\
$\mathrm{C}$ & -2.31365600 & -0.21371200 & -1.15967200 \\
$\mathrm{C}$ & -3.69401300 & -0.19730300 & -1.27697400 \\
$\mathrm{C}$ & -4.46842100 & -0.59100700 & -0.19189300 \\
$\mathrm{C}$ & -3.88386700 & -0.99695100 & 1.00158700 \\
$\mathrm{C}$ & -2.50181700 & -1.01157200 & 1.11042500 \\
$\mathrm{C}$ & 6.19280100 & -1.49973300 & -1.23451700 \\
$\mathrm{C}$ & 6.98857100 & -1.24625500 & -0.14575000 \\
$\mathrm{C}$ & 6.40698700 & -0.84655100 & 1.07137900 \\
$\mathrm{C}$ & 5.04422900 & -0.71877100 & 1.17863800 \\
$\mathrm{O}$ & 0.46024400 & 1.45722300 & 1.69813000 \\
$\mathrm{C}$ & 0.91630100 & 2.28566500 & 0.68577000 \\
$\mathrm{C}$ & 2.24681000 & 2.68416100 & 0.66989800 \\
$\mathrm{C}$ & 2.68261600 & 3.55665600 & -0.32038400
\end{tabular}




$\begin{array}{lrrr}\mathrm{C} & 1.79498500 & 4.02875600 & -1.28129000 \\ \mathrm{C} & 0.46349100 & 3.62804200 & -1.24829400 \\ \mathrm{C} & 0.01770000 & 2.75316200 & -0.26443600 \\ \mathrm{H} & 4.43258700 & -1.91538000 & -3.22477200 \\ \mathrm{H} & 1.98678800 & -1.67957900 & -3.06221500 \\ \mathrm{H} & 2.74033300 & -0.56487100 & 2.17220200 \\ \mathrm{H} & 0.04252500 & -1.34438700 & -1.84067400 \\ \mathrm{H} & -1.70705900 & 0.11885100 & -1.99548900 \\ \mathrm{H} & -4.50381500 & -1.32062800 & 1.83043400 \\ \mathrm{H} & -2.03891300 & -1.33692000 & 2.03490500 \\ \mathrm{H} & 6.63552900 & -1.80026600 & -2.17963700 \\ \mathrm{H} & 8.06645200 & -1.34661600 & -0.22195500 \\ \mathrm{H} & 7.03708900 & -0.63298600 & 1.92842700 \\ \mathrm{H} & 4.63952900 & -0.38917000 & 2.13049200 \\ \mathrm{H} & 2.92574600 & 2.31728300 & 1.43179900 \\ \mathrm{H} & 3.72150800 & 3.87004000 & -0.33630400 \\ \mathrm{H} & 2.13891500 & 4.71194800 & -2.05096800 \\ \mathrm{H} & -0.23621600 & 3.99911000 & -1.99047800 \\ \mathrm{H} & -1.01902700 & 2.43708400 & -0.21872100 \\ \mathrm{H} & -4.16844000 & 0.11128900 & -2.20190900 \\ \mathrm{~S} & -6.24336400 & -0.55716900 & -0.33035900 \\ \mathrm{O} & -6.58686700 & -0.61833800 & -1.74964100 \\ \mathrm{O} & -6.79035100 & -1.55176400 & 0.58960200 \\ \mathrm{C} & -6.69337200 & 1.05737900 & 0.27475200 \\ \mathrm{H} & -6.37399500 & 1.15321000 & 1.31148500 \\ \mathrm{H} & -6.22679700 & 1.81685100 & -0.35099200 \\ \mathrm{H} & -7.77987100 & 1.12182200 & 0.20457700\end{array}$

Table S11. Cartesian coordinates for compound $8 d\left(S_{1}\right)$.

\begin{tabular}{rrrr}
\hline & & $\mathrm{Y}$ & $\mathrm{Z}$ \\
\hline $\mathrm{C}$ & 4.07929300 & -1.39921200 & -2.30775000 \\
$\mathrm{C}$ & 2.70727300 & -1.37003800 & -2.21310600 \\
$\mathrm{C}$ & 4.90283500 & -1.14432900 & -1.17971300 \\
$\mathrm{C}$ & 4.28109000 & -0.85592900 & 0.07474400 \\
$\mathrm{C}$ & 2.86031400 & -0.85963100 & 0.15554600 \\
$\mathrm{C}$ & 2.04059100 & -1.09501500 & -1.00392900 \\
$\mathrm{~N}$ & 2.22702600 & -0.68363100 & 1.34765700 \\
$\mathrm{P}$ & 0.58374000 & -0.35339700 & 1.63765300 \\
$\mathrm{C}$ & -0.20967700 & -0.81846200 & 0.12381300 \\
$\mathrm{C}$ & 0.62458800 & -1.10059100 & -0.96690900 \\
$\mathrm{O}$ & 0.17718800 & -0.91542000 & 2.94284300 \\
$\mathrm{C}$ & -1.64340600 & -0.75679900 & 0.04033600 \\
$\mathrm{C}$ & -2.31346900 & -0.83946200 & -1.21332700 \\
$\mathrm{C}$ & -3.68582600 & -0.77385600 & -1.30330900 \\
$\mathrm{C}$ & -4.45541100 & -0.62786700 & -0.14561700 \\
$\mathrm{C}$ & -3.83378200 & -0.54771400 & 1.10643400 \\
$\mathrm{C}$ & -2.46116900 & -0.61517400 & 1.19552500 \\
$\mathrm{C}$ & 6.29934100 & -1.16340600 & -1.27716500
\end{tabular}




$\begin{array}{lrrr}\mathrm{C} & 7.09951900 & -0.90249600 & -0.17335400 \\ \mathrm{C} & 6.49806500 & -0.60712800 & 1.05217200 \\ \mathrm{C} & 5.11875500 & -0.58068400 & 1.17700100 \\ \mathrm{O} & 0.50417300 & 1.27234800 & 1.74835600 \\ \mathrm{C} & 0.79511300 & 2.14744800 & 0.71493900 \\ \mathrm{C} & 2.08531000 & 2.64647000 & 0.59003500 \\ \mathrm{C} & 2.36157800 & 3.56693500 & -0.41490000 \\ \mathrm{C} & 1.35664100 & 3.98148000 & -1.28161100 \\ \mathrm{C} & 0.06768400 & 3.47641100 & -1.13979100 \\ \mathrm{C} & -0.22029500 & 2.55552000 & -0.14023200 \\ \mathrm{H} & 4.54900600 & -1.61993400 & -3.26102800 \\ \mathrm{H} & 2.10719400 & -1.57338300 & -3.09431100 \\ \mathrm{H} & 2.78594900 & -0.70073700 & 2.19020600 \\ \mathrm{H} & 0.15240900 & -1.36476700 & -1.90676300 \\ \mathrm{H} & -1.74839800 & -0.93719900 & -2.13227200 \\ \mathrm{H} & -4.43478500 & -0.46327300 & 2.00544100 \\ \mathrm{H} & -2.00458900 & -0.59261600 & 2.17881400 \\ \mathrm{H} & 6.75253900 & -1.38622800 & -2.23855100 \\ \mathrm{H} & 8.17987800 & -0.92258000 & -0.26270300 \\ \mathrm{H} & 7.11271300 & -0.39041600 & 1.91959300 \\ \mathrm{H} & 4.70434400 & -0.32303200 & 2.14672400 \\ \mathrm{H} & 2.85611000 & 2.32210600 & 1.28116000 \\ \mathrm{H} & 3.36721600 & 3.96224900 & -0.51559100 \\ \mathrm{H} & 1.57561300 & 4.70147700 & -2.06332900 \\ \mathrm{H} & -0.72197900 & 3.80185900 & -1.80936100 \\ \mathrm{H} & -1.22011100 & 2.15536000 & -0.01213700 \\ \mathrm{H} & -4.17446400 & -0.84659600 & -2.26915700 \\ \mathrm{~S} & -6.20778700 & -0.50332800 & -0.27156500 \\ \mathrm{O} & -6.62821900 & -1.13303300 & -1.52489200 \\ \mathrm{O} & -6.79911100 & -0.94812900 & 0.99138100 \\ \mathrm{C} & -6.53289400 & 1.24566500 & -0.42239900 \\ \mathrm{H} & -6.16093100 & 1.75662400 & 0.46467500 \\ \mathrm{H} & -6.04775600 & 1.62258700 & -1.32177100 \\ \mathrm{H} & -7.61525400 & 1.35916700 & -0.49908300\end{array}$

Table S12. Cartesian coordinates for compound $8 \mathrm{e}\left(\mathrm{S}_{0}\right)$.

\begin{tabular}{lrrr}
\hline & $\mathrm{X}$ & $\mathrm{Y}$ & $\mathrm{Z}$ \\
$\mathrm{C}$ & 3.75052200 & -1.60949100 & -2.29428600 \\
$\mathrm{C}$ & 2.39947300 & -1.48491300 & -2.19599600 \\
$\mathrm{C}$ & 4.57549900 & -1.35611000 & -1.16680700 \\
$\mathrm{C}$ & 3.98694600 & -0.96720100 & 0.07107400 \\
$\mathrm{C}$ & 2.56169200 & -0.86199200 & 0.14698000 \\
$\mathrm{C}$ & 1.77282100 & -1.10909200 & -0.97666400 \\
$\mathrm{~N}$ & 1.95759000 & -0.53095900 & 1.34225000 \\
$\mathrm{P}$ & 0.34631700 & -0.17499200 & 1.64759200 \\
$\mathrm{C}$ & -0.43632300 & -0.66757800 & 0.13119300 \\
$\mathrm{C}$ & 0.34053800 & -1.03676200 & -0.92003600 \\
$\mathrm{O}$ & -0.12714900 & -0.70992600 & 2.94290400 \\
$\mathrm{C}$ & -1.90834000 & -0.61878900 & 0.03654800
\end{tabular}




$\begin{array}{lrrr}\text { C } & -2.52564500 & -0.21707800 & -1.15613800 \\ \mathrm{C} & -3.90524700 & -0.19561400 & -1.27153800 \\ \mathrm{C} & -4.69823500 & -0.55947400 & -0.18668400 \\ \mathrm{C} & -4.10257900 & -0.94118500 & 1.01001400 \\ \mathrm{C} & -2.72049800 & -0.96792100 & 1.12283500 \\ \mathrm{C} & 5.98076000 & -1.47463200 & -1.24921400 \\ \mathrm{C} & 6.78013400 & -1.21603100 & -0.16433500 \\ \mathrm{C} & 6.20184500 & -0.81728600 & 1.05469700 \\ \mathrm{C} & 4.83900600 & -0.69490400 & 1.16734900 \\ \mathrm{O} & 0.23011000 & 1.45034900 & 1.73373800 \\ \mathrm{C} & 0.60607300 & 2.29914500 & 0.70532500 \\ \mathrm{C} & 1.93248700 & 2.69236600 & 0.58176300 \\ \mathrm{C} & 2.28444100 & 3.58678000 & -0.42257300 \\ \mathrm{C} & 1.31865800 & 4.08652100 & -1.28937500 \\ \mathrm{C} & -0.00738900 & 3.69086100 & -1.14812600 \\ \mathrm{C} & -0.36970200 & 2.79346600 & -0.15051600 \\ \mathrm{H} & 4.21369900 & -1.90245500 & -3.23099700 \\ \mathrm{H} & 1.76742300 & -1.67805200 & -3.05772600 \\ \mathrm{H} & 2.53925900 & -0.53615100 & 2.16778600 \\ \mathrm{H} & -0.17255900 & -1.33914300 & -1.83176900 \\ \mathrm{H} & -1.91905200 & 0.10851400 & -1.99465500 \\ \mathrm{H} & -4.36506100 & 0.12562800 & -2.19974600 \\ \mathrm{H} & -4.71639600 & -1.21639500 & 1.86038600 \\ \mathrm{H} & -2.26436900 & -1.27040100 & 2.05847100 \\ \mathrm{H} & 6.42072500 & -1.77498900 & -2.19571000 \\ \mathrm{H} & 7.85811300 & -1.31196100 & -0.24493000 \\ \mathrm{H} & 6.83447600 & -0.60043600 & 1.90906800 \\ \mathrm{H} & 4.43705000 & -0.36671200 & 2.12084000 \\ \mathrm{H} & 2.67436500 & 2.30549600 & 1.27151800 \\ \mathrm{H} & 3.31986800 & 3.89623700 & -0.52249600 \\ \mathrm{H} & 1.59775600 & 4.78753200 & -2.06922600 \\ \mathrm{H} & -0.76806100 & 4.08335500 & -1.81552200 \\ \mathrm{H} & -1.40005400 & 2.48007300 & -0.02114300 \\ \mathrm{C} & -6.18732900 & -0.57974600 & -0.32989000 \\ \mathrm{~F} & -6.62433800 & -1.74833300 & -0.83771700 \\ \mathrm{~F} & -6.81444800 & -0.41329200 & 0.84359400 \\ \mathrm{~F} & -6.62748100 & 0.38252100 & -1.15704700\end{array}$

Table S13. Cartesian coordinates for compound $8 \mathrm{e}\left(\mathrm{S}_{1}\right)$.

\begin{tabular}{lrrr}
\hline & $\mathrm{X}$ & $\mathrm{Y}$ & $\mathrm{Z}$ \\
$\mathrm{C}$ & 3.84892700 & -1.43999300 & -2.28621400 \\
$\mathrm{C}$ & 2.47822400 & -1.40540800 & -2.20172500 \\
$\mathrm{C}$ & 4.66799700 & -1.16792000 & -1.15648200 \\
$\mathrm{C}$ & 4.03778800 & -0.85870100 & 0.08947500 \\
$\mathrm{C}$ & 2.61870400 & -0.86700700 & 0.16356700 \\
$\mathrm{C}$ & 1.80258100 & -1.10977400 & -0.99998600 \\
$\mathrm{~N}$ & 1.97945400 & -0.69564400 & 1.35513800 \\
$\mathrm{P}$ & 0.33859800 & -0.34656100 & 1.63333400 \\
$\mathrm{C}$ & -0.44741900 & -0.79836500 & 0.11551600
\end{tabular}




$\begin{array}{lrrr}\mathrm{C} & 0.38981700 & -1.10357200 & -0.97043100 \\ \mathrm{O} & -0.08233100 & -0.90319200 & 2.93730200 \\ \mathrm{C} & -1.87939200 & -0.69964100 & 0.01292300 \\ \mathrm{C} & -2.53223800 & -0.71345900 & -1.24901300 \\ \mathrm{C} & -3.90474600 & -0.62566400 & -1.35178400 \\ \mathrm{C} & -4.69824100 & -0.51260300 & -0.20871000 \\ \mathrm{C} & -4.08197200 & -0.48948600 & 1.04745600 \\ \mathrm{C} & -2.71147200 & -0.58152700 & 1.15792700 \\ \mathrm{C} & 6.06373600 & -1.18964700 & -1.24429000 \\ \mathrm{C} & 6.85835000 & -0.90981400 & -0.13937200 \\ \mathrm{C} & 6.24943300 & -0.58992500 & 1.07615100 \\ \mathrm{C} & 4.86954400 & -0.56039600 & 1.19215900 \\ \mathrm{O} & 0.27638800 & 1.28166400 & 1.74785800 \\ \mathrm{C} & 0.55752200 & 2.15209400 & 0.70856800 \\ \mathrm{C} & 1.85743900 & 2.61006300 & 0.53510600 \\ \mathrm{C} & 2.12480400 & 3.52494500 & -0.47734400 \\ \mathrm{C} & 1.10128300 & 3.97577900 & -1.30315700 \\ \mathrm{C} & -0.19714600 & 3.51247200 & -1.11260100 \\ \mathrm{C} & -0.47601200 & 2.59658300 & -0.10594200 \\ \mathrm{H} & 4.32477500 & -1.67632600 & -3.23283900 \\ \mathrm{H} & 1.88288700 & -1.61912000 & -3.08386800 \\ \mathrm{H} & 2.53141000 & -0.73643300 & 2.20144600 \\ \mathrm{H} & -0.08386300 & -1.37753400 & -1.90720800 \\ \mathrm{H} & -1.95315200 & -0.76088400 & -2.16344700 \\ \mathrm{H} & -4.36807300 & -0.62773600 & -2.33269900 \\ \mathrm{H} & -4.68432600 & -0.40185700 & 1.94568900 \\ \mathrm{H} & -2.26837200 & -0.59043000 & 2.14767000 \\ \mathrm{H} & 6.52313200 & -1.42926300 & -2.19881000 \\ \mathrm{H} & 7.93925000 & -0.93239400 & -0.22188100 \\ \mathrm{H} & 6.85901600 & -0.35442800 & 1.94241900 \\ \mathrm{H} & 4.44698000 & -0.27590400 & 2.15090700 \\ \mathrm{H} & 2.64361700 & 2.25751900 & 1.19420800 \\ \mathrm{H} & 3.13832000 & 3.88742500 & -0.61587500 \\ \mathrm{H} & 1.31326100 & 4.69162300 & -2.09065400 \\ \mathrm{H} & -1.00149600 & 3.86642000 & -1.74943300 \\ \mathrm{H} & -1.48264500 & 2.22853600 & 0.05931100 \\ \mathrm{C} & -6.18173000 & -0.47704400 & -0.31424500 \\ \mathrm{~F} & -6.74052100 & -1.69688300 & -0.14852000 \\ \mathrm{~F} & -6.74149800 & 0.31466100 & 0.61898000 \\ & -6.60054000 & -0.03344600 & -1.51240400\end{array}$

Table S14. Cartesian coordinates for compound $8 f\left(\mathrm{~S}_{0}\right)$.

\begin{tabular}{lrrr}
\hline & $\mathrm{X}$ & $\mathrm{Y}$ & $\mathrm{Z}$ \\
$\mathrm{C}$ & 3.28337400 & -1.48620000 & -2.34080100 \\
$\mathrm{C}$ & 1.92832000 & -1.42638200 & -2.22726100 \\
$\mathrm{C}$ & 4.10662100 & -1.22178300 & -1.21539400 \\
$\mathrm{C}$ & 3.51142800 & -0.88939300 & 0.03599700 \\
$\mathrm{C}$ & 2.08404600 & -0.84966700 & 0.12528100 \\
$\mathrm{C}$ & 1.29585800 & -1.10720400 & -0.99556300
\end{tabular}




\begin{tabular}{lrrr}
$\mathrm{N}$ & 1.47473900 & -0.57186000 & 1.33286100 \\
$\mathrm{P}$ & -0.14654200 & -0.27803800 & 1.64979600 \\
$\mathrm{C}$ & -0.92500200 & -0.78517600 & 0.13633300 \\
$\mathrm{C}$ & -0.13996700 & -1.10323600 & -0.92501000 \\
$\mathrm{O}$ & -0.58870100 & -0.83728700 & 2.94650200 \\
$\mathrm{C}$ & -2.39779300 & -0.78109800 & 0.05973500 \\
$\mathrm{C}$ & -3.04840000 & -0.37688500 & -1.10923400 \\
$\mathrm{C}$ & -4.43313100 & -0.39593400 & -1.21977800 \\
$\mathrm{C}$ & -5.20771600 & -0.81677100 & -0.13791700 \\
$\mathrm{C}$ & -4.57247200 & -1.20612000 & 1.04469100 \\
$\mathrm{C}$ & -3.19468500 & -1.18580500 & 1.14311600 \\
$\mathrm{C}$ & 5.51529400 & -1.27454100 & -1.31125500 \\
$\mathrm{C}$ & 6.31203900 & -1.00686200 & -0.22679900 \\
$\mathrm{C}$ & 5.72660700 & -0.66569100 & 1.00646900 \\
$\mathrm{C}$ & 4.36068800 & -0.60765300 & 1.13239500 \\
$\mathrm{O}$ & -0.31874400 & 1.34398900 & 1.75327200 \\
$\mathrm{C}$ & 0.05390900 & 2.21696800 & 0.74610400 \\
$\mathrm{C}$ & 1.35536100 & 2.70081100 & 0.70105700 \\
$\mathrm{C}$ & 1.70486500 & 3.62095000 & -0.28050300 \\
$\mathrm{C}$ & 0.76085700 & 4.05547000 & -1.20471700 \\
$\mathrm{C}$ & -0.54042600 & 3.56778800 & -1.14378400 \\
$\mathrm{C}$ & -0.89989600 & 2.64506300 & -0.16843000 \\
$\mathrm{H}$ & 3.74994100 & -1.73623900 & -3.28837900 \\
$\mathrm{H}$ & 1.29798300 & -1.62952900 & -3.08812500 \\
$\mathrm{H}$ & 2.06304300 & -0.56034800 & 2.15325800 \\
$\mathrm{H}$ & -0.64771400 & -1.41186500 & -1.83782400 \\
$\mathrm{H}$ & -2.46543400 & -0.01357300 & -1.95016800 \\
$\mathrm{H}$ & -4.89275100 & -0.06723400 & -2.14429600 \\
$\mathrm{H}$ & -5.18491500 & -1.52778800 & 1.88057800 \\
$\mathrm{H}$ & -2.72205800 & -1.49831800 & 2.06771900 \\
$\mathrm{H}$ & 5.95984000 & -1.53195100 & -2.26831400 \\
$\mathrm{H}$ & 7.39254300 & -1.05211500 & -0.31800900 \\
$\mathrm{H}$ & 6.35615700 & -0.44312000 & 1.86176200 \\
$\mathrm{H}$ & 3.95253200 & -0.32525100 & 2.09788200 \\
$\mathrm{H}$ & -6.96364300 & -1.12804500 & -2.15869700 \\
$\mathrm{H}$ & -8.29926000 & -0.61869400 & -1.09740400 \\
$\mathrm{H}$ & & & \\
$\mathrm{H}$ & 2.72080500 & 4.00093800 & -0.31844600 \\
$\mathrm{H}$ & 1.03739400 & 4.77629200 & -1.96730600 \\
$\mathrm{H}$ & -1.28430400 & 3.90779900 & -1.85745600 \\
\hline & -1.91131000 & 2.25874600 & -0.10202900 \\
$\mathrm{H}$ & -6.55678200 & -0.87206200 & -0.13558600 \\
$\mathrm{H}$ & -7.24012800 & -0.49104600 & -1.31218900 \\
$\mathrm{H}$ & & &
\end{tabular}

Table S15. Cartesian coordinates for compound $8 \mathbf{8}\left(\mathrm{S}_{1}\right)$.

\begin{tabular}{ccccc}
\hline & $\mathrm{X}$ & $\mathrm{Y}$ & $\mathrm{Z}$ \\
$\mathrm{C}$ & 3.35589900 & -1.75743400 & -2.12240200 \\
$\mathrm{C}$ & 2.00324700 & -1.78527700 & -2.00823000
\end{tabular}




$\begin{array}{lrrr}\mathrm{C} & 4.17978800 & -1.23398500 & -1.07463100 \\ \mathrm{C} & 3.55341300 & -0.74128900 & 0.11500200 \\ \mathrm{C} & 2.14714300 & -0.82146600 & 0.23543600 \\ \mathrm{C} & 1.32697600 & -1.31131400 & -0.84286200 \\ \mathrm{~N} & 1.53299200 & -0.49011300 & 1.42111700 \\ \mathrm{P} & -0.10696100 & -0.21810600 & 1.70507600 \\ \mathrm{C} & -0.89472400 & -0.89305000 & 0.27886600 \\ \mathrm{C} & -0.06603200 & -1.35840400 & -0.77455400 \\ \mathrm{O} & -0.48381900 & -0.63019200 & 3.07786600 \\ \mathrm{C} & -2.32251300 & -0.82192200 & 0.16662200 \\ \mathrm{C} & -2.98782800 & -1.01810400 & -1.07454800 \\ \mathrm{C} & -4.36220200 & -0.95568000 & -1.19045200 \\ \mathrm{C} & -5.15045100 & -0.68290800 & -0.06457600 \\ \mathrm{C} & -4.52036900 & -0.47670800 & 1.17686900 \\ \mathrm{C} & -3.15588900 & -0.54825200 & 1.29058100 \\ \mathrm{C} & 5.57030900 & -1.19156100 & -1.19013700 \\ \mathrm{C} & 6.36508800 & -0.67431400 & -0.17378600 \\ \mathrm{C} & 5.76010000 & -0.17255200 & 0.98372500 \\ \mathrm{C} & 4.38801400 & -0.19901400 & 1.12912300 \\ \mathrm{O} & -0.30251000 & 1.40837500 & 1.63725700 \\ \mathrm{C} & -0.05533800 & 2.17215100 & 0.51225400 \\ \mathrm{C} & 1.22527100 & 2.65813100 & 0.27882500 \\ \mathrm{C} & 1.45376700 & 3.47070500 & -0.82570600 \\ \mathrm{C} & 0.41038400 & 3.79641800 & -1.68530000 \\ \mathrm{C} & -0.86861100 & 3.30884000 & -1.43568000 \\ \mathrm{C} & -1.10764700 & 2.49329700 & -0.33641200 \\ \mathrm{H} & 3.83604200 & -2.13005200 & -3.02247400 \\ \mathrm{H} & -7.39699200 & -2.17875400 & -2.81905600 \\ \mathrm{H} & -8.23599400 & -0.68104400 & -1.05054600 \\ \mathrm{H} & 2.10565100 & -0.46700900 & 2.25390300 \\ \mathrm{H} & -0.55643600 & -1.81983700 & -1.62614500 \\ \mathrm{H} & -2.41198500 & -1.19445900 & -1.97525200 \\ \mathrm{H} & -4.81562600 & -1.11095700 & -2.16246100 \\ \mathrm{H} & -5.14150400 & -0.27485300 & 2.04322100 \\ \mathrm{H} & -2.70610700 & -0.41951800 & 2.26936100 \\ \mathrm{H} & 6.02984400 & -1.57209300 & -2.09827400 \\ \mathrm{H} & 7.44434200 & -0.65083500 & -0.28157300 \\ \mathrm{H} & 6.37158300 & 0.25212000 & 1.77387600 \\ \mathrm{H} & 3.95815400 & 0.23661300 & 2.02587100 \\ \mathrm{H} & 2.02749800 & 2.40180700 & 0.96234300 \\ \mathrm{H} & 2.45321600 & 3.85095400 & -1.01131500 \\ \mathrm{H} & 0.59207900 & 4.43292900 & -2.54528500 \\ \mathrm{H} & -1.68862300 & 3.56476400 & -2.09927300 \\ \mathrm{H} & -6.48860700 & -0.59929300 & -0.06657500 \\ \mathrm{H} & -7.18044300 & -0.79497400 & -1.28655000 \\ \mathrm{H} & -6.88962200 & -0.04598800 & -2.02935200 \\ \mathrm{H} & & -1.79829100 & -1.68475700 \\ \mathrm{H} & -1.00800 & \\ \mathrm{H} & & & \end{array}$


Table S16. Cartesian coordinates for compund 7a.

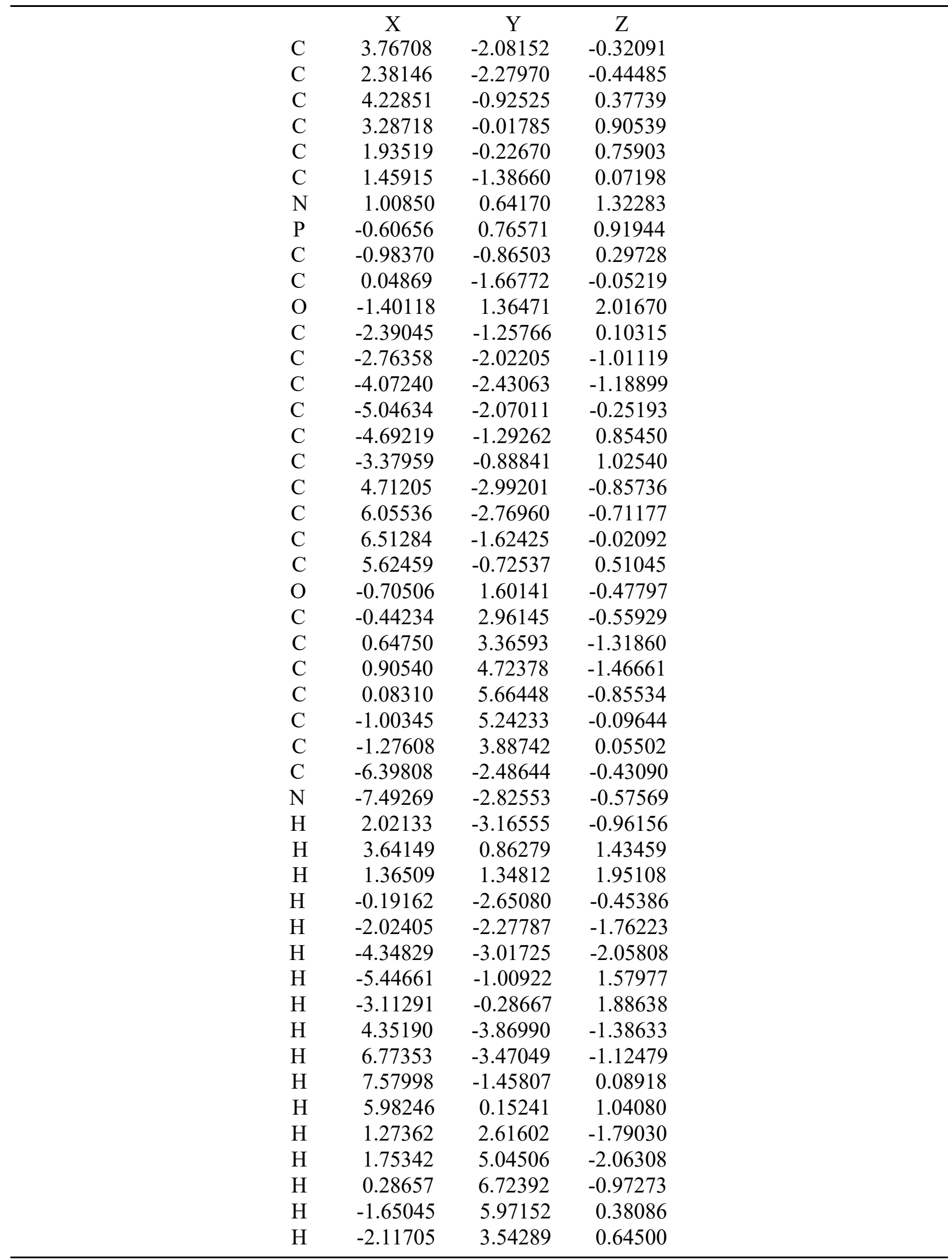


Table S17. Cartesian coordinates for compund 7b.

\begin{tabular}{|c|c|c|c|}
\hline & $\mathrm{X}$ & Y & $\mathrm{Z}$ \\
\hline $\mathrm{C}$ & 4.00277 & -2.51575 & -0.32109 \\
\hline $\mathrm{C}$ & 2.60051 & -2.50152 & -0.41789 \\
\hline $\mathrm{C}$ & 4.64924 & -1.43132 & 0.34491 \\
\hline $\mathrm{C}$ & 3.86766 & -0.38169 & 0.86954 \\
\hline $\mathrm{C}$ & 2.49695 & -0.38484 & 0.75167 \\
\hline $\mathrm{C}$ & 1.83549 & -1.46951 & 0.09610 \\
\hline $\mathrm{N}$ & 1.72651 & 0.62452 & 1.31544 \\
\hline $\mathrm{P}$ & 0.14035 & 0.98634 & 0.94161 \\
\hline $\mathrm{C}$ & -0.49386 & -0.57584 & 0.35470 \\
\hline $\mathrm{C}$ & 0.39545 & -1.53278 & 0.00219 \\
\hline $\mathrm{O}$ & -0.52803 & 1.71594 & 2.04406 \\
\hline $\mathrm{C}$ & -1.94942 & -0.74774 & 0.19154 \\
\hline $\mathrm{C}$ & -2.46037 & -1.44582 & -0.90980 \\
\hline $\mathrm{C}$ & -3.82308 & -1.64863 & -1.05296 \\
\hline $\mathrm{C}$ & -4.70261 & -1.14753 & -0.09821 \\
\hline $\mathrm{C}$ & -4.21280 & -0.43616 & 0.99201 \\
\hline C & -2.84884 & -0.23441 & 1.13484 \\
\hline C & 4.78731 & -3.56960 & -0.85342 \\
\hline $\mathrm{C}$ & 6.15154 & -3.55294 & -0.73424 \\
\hline $\mathrm{C}$ & 6.79211 & -2.47877 & -0.07546 \\
\hline $\mathrm{C}$ & 6.06184 & -1.44487 & 0.45078 \\
\hline $\mathrm{O}$ & 0.14263 & 1.81223 & -0.46606 \\
\hline $\mathrm{C}$ & 0.62974 & 3.10750 & -0.56554 \\
\hline $\mathrm{C}$ & 1.76644 & 3.31554 & -1.33541 \\
\hline C & 2.24982 & 4.60870 & -1.49742 \\
\hline C & 1.60374 & 5.68008 & -0.88966 \\
\hline $\mathrm{C}$ & 0.46744 & 5.45428 & -0.11975 \\
\hline $\mathrm{C}$ & -0.02930 & 4.16639 & 0.04630 \\
\hline $\mathrm{H}$ & 2.09902 & -3.33091 & -0.90988 \\
\hline $\mathrm{H}$ & 4.36110 & 0.44475 & 1.37405 \\
\hline $\mathrm{H}$ & 2.20080 & 1.27642 & 1.92446 \\
\hline $\mathrm{H}$ & -0.00171 & -2.47252 & -0.37840 \\
\hline $\mathrm{H}$ & -1.78593 & -1.81402 & -1.67558 \\
\hline $\mathrm{H}$ & -4.20174 & -2.19240 & -1.91127 \\
\hline $\mathrm{H}$ & -4.89543 & -0.04648 & 1.73908 \\
\hline $\mathrm{H}$ & -2.47404 & 0.31683 & 1.98942 \\
\hline $\mathrm{H}$ & 4.28760 & -4.39176 & -1.35800 \\
\hline $\mathrm{H}$ & 6.74586 & -4.36317 & -1.14405 \\
\hline $\mathrm{H}$ & 7.87404 & -2.47610 & 0.01383 \\
\hline $\mathrm{H}$ & 6.55972 & -0.62247 & 0.95647 \\
\hline $\mathrm{H}$ & 2.25334 & 2.46638 & -1.80319 \\
\hline $\mathrm{H}$ & 3.13554 & 4.77663 & -2.10196 \\
\hline $\mathrm{H}$ & 1.98306 & 6.68871 & -1.01775 \\
\hline $\mathrm{H}$ & -0.04254 & 6.28630 & 0.35549 \\
\hline $\mathrm{H}$ & -0.91088 & 3.97354 & 0.64594 \\
\hline $\mathrm{C}$ & -6.17834 & -1.31857 & -0.27662 \\
\hline $\mathrm{F}$ & -6.82390 & -1.39472 & 0.89756 \\
\hline $\mathrm{F}$ & -6.47910 & -2.42850 & -0.96893 \\
\hline $\mathrm{F}$ & -6.72501 & -0.28757 & -0.94839 \\
\hline
\end{tabular}


Table S18. Cartesian coordinates for compund 7c.

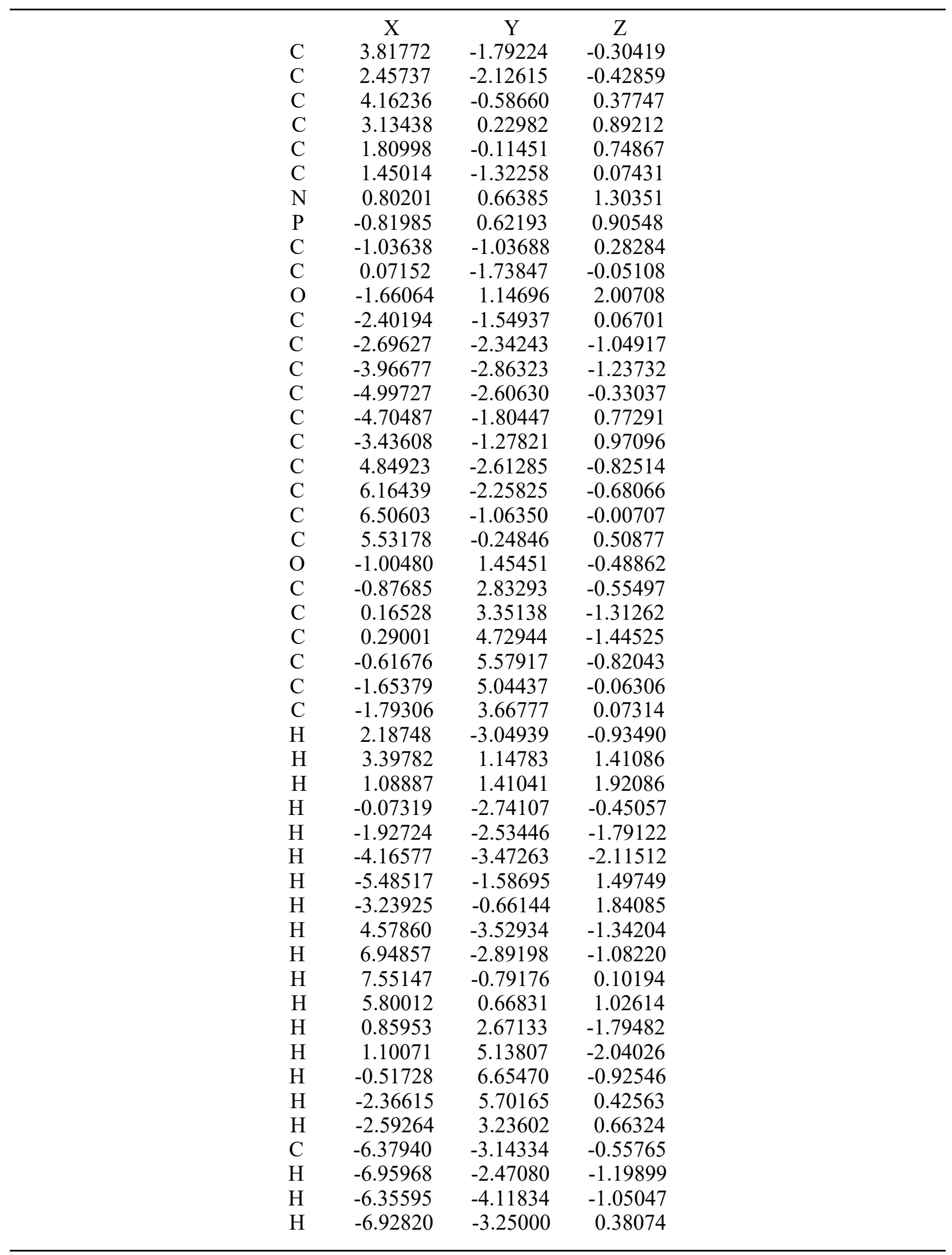


Table S19. Cartesian coordinates for compund 7d.

\begin{tabular}{|c|c|c|c|c|}
\hline & & X & $\mathrm{Y}$ & Z \\
\hline & $\mathrm{C}$ & 3.74075 & -2.24608 & -0.31603 \\
\hline & $\mathrm{C}$ & 2.34191 & -2.36980 & -0.39278 \\
\hline & $\mathrm{C}$ & 4.28755 & -1.09827 & 0.33198 \\
\hline & $\mathrm{C}$ & 3.41297 & -0.12544 & 0.85843 \\
\hline & $\mathrm{C}$ & 2.04778 & -0.26436 & 0.75998 \\
\hline & $\mathrm{C}$ & 1.48544 & -1.41439 & 0.12380 \\
\hline & $\mathrm{N}$ & 1.18862 & 0.67028 & 1.32466 \\
\hline $\mathrm{P}$ & $P$ & -0.42983 & 0.87188 & 0.96326 \\
\hline $\mathrm{C}$ & $\mathrm{C}$ & -0.92114 & -0.75039 & 0.40282 \\
\hline $\mathrm{C}$ & $\mathrm{C}$ & 0.05617 & -1.61982 & 0.05481 \\
\hline & $\mathrm{O}$ & -1.14768 & 1.55591 & 2.06462 \\
\hline $\mathrm{C}$ & $\mathrm{C}$ & -2.35434 & -1.04978 & 0.24341 \\
\hline $\mathrm{C}$ & $\mathrm{C}$ & -2.80655 & -1.81359 & -0.83563 \\
\hline $\mathrm{C}$ & $\mathrm{C}$ & -4.14691 & -2.14433 & -0.98598 \\
\hline $\mathrm{C}$ & $\mathrm{C}$ & -5.07837 & -1.70099 & -0.04599 \\
\hline $\mathrm{C}$ & $\mathrm{C}$ & -4.64412 & -0.92038 & 1.02931 \\
\hline $\mathrm{C}$ & $\mathrm{C}$ & -3.30870 & -0.59674 & 1.16902 \\
\hline C & $\mathrm{C}$ & 4.61775 & -3.22241 & -0.85133 \\
\hline & $\mathrm{C}$ & 5.97573 & -3.07185 & -0.75207 \\
\hline $\mathrm{C}$ & $\mathrm{C}$ & 6.51749 & -1.93496 & -0.11061 \\
\hline & $\mathrm{C}$ & 5.69596 & -0.97295 & 0.41796 \\
\hline $\mathrm{O}$ & $\mathrm{O}$ & -0.51958 & 1.67848 & -0.45546 \\
\hline & $\mathrm{C}$ & -0.18180 & 3.01659 & -0.57963 \\
\hline & $\mathrm{C}$ & 0.90223 & 3.33846 & -1.38606 \\
\hline & $\mathrm{C}$ & 1.23401 & 4.67446 & -1.57870 \\
\hline & $\mathrm{C}$ & 0.49094 & 5.67716 & -0.96459 \\
\hline C & $\mathrm{C}$ & -0.59022 & 5.33846 & -0.15754 \\
\hline & $\mathrm{C}$ & -0.93613 & 4.00626 & 0.03899 \\
\hline & $\mathrm{H}$ & 1.91831 & -3.24918 & -0.87117 \\
\hline & $\mathrm{H}$ & 3.82897 & 0.75041 & 1.34915 \\
\hline & $\mathrm{H}$ & 1.60526 & 1.37775 & 1.91366 \\
\hline $\mathrm{H}$ & $\mathrm{H}$ & -0.25137 & -2.59875 & -0.31007 \\
\hline $\mathrm{H}$ & $\mathrm{H}$ & -2.10257 & -2.13879 & -1.59532 \\
\hline $\mathrm{H}$ & $\mathrm{H}$ & -4.45181 & -2.73081 & -1.84434 \\
\hline & $\mathrm{H}$ & -5.37833 & -0.57778 & 1.75097 \\
\hline & $\mathrm{H}$ & -2.99303 & 0.00758 & 2.01209 \\
\hline & $\mathrm{H}$ & 4.19418 & -4.09410 & -1.34251 \\
\hline & $\mathrm{H}$ & 6.64048 & -3.82417 & -1.16436 \\
\hline & $\mathrm{H}$ & 7.59510 & -1.82555 & -0.03665 \\
\hline & $\mathrm{H}$ & 6.11724 & -0.10101 & 0.91027 \\
\hline & $\mathrm{H}$ & 1.46643 & 2.54120 & -1.85833 \\
\hline & $\mathrm{H}$ & 2.07770 & 4.92997 & -2.21211 \\
\hline & $\mathrm{H}$ & 0.75171 & 6.71956 & -1.11666 \\
\hline & $\mathrm{H}$ & -1.17570 & 6.11614 & 0.32281 \\
\hline & $\mathrm{H}$ & -1.77314 & 3.72656 & 0.66753 \\
\hline $\mathrm{O}$ & $\mathrm{O}$ & -6.40189 & -1.96224 & -0.09479 \\
\hline $\mathrm{C}$ & $\mathrm{C}$ & -6.88669 & -2.75287 & -1.16104 \\
\hline $\mathrm{H}$ & $\mathrm{H}$ & -7.95950 & -2.84285 & -1.00229 \\
\hline $\mathrm{H}$ & $\mathrm{H}$ & -6.70424 & -2.27474 & -2.12890 \\
\hline & $\mathrm{H}$ & -6.43581 & -3.75061 & -1.15556 \\
\hline
\end{tabular}




\subsection{Dimerization}

The geometries for 8a-f and their dimeric complexes were fully optimized without symmetry constraint by using the functional M06- $2 \mathrm{X}^{10}$ (accounting for the contributions of H-bond/ dispersion forces) and TZVP basis set ${ }^{11}$ as implemented in Gaussian 09. The PCM solvation model was used to account for the effects of the chloroform environment. All of the optimized structures were confirmed by frequency calculations to be minima using the same level of theory. The solution-phase optimized geometries were used in all the energy calculations. Single point calculations were computed at a higher level using the minimally diffuse-augmented ma-def2TZVP (ma-TZVP) basis set $^{12}$ (obtained from https://comp.chem.umn.edu/basissets/basis.cgi). The interaction energy ( $\left.\Delta E_{\text {int }}\right)$ is defined as the gas-phase electronic energy difference between dimer and its isolated monomers (The enantiomers have exactly the same electronic energies), and the basis set superposition error (BSSE) were corrected by the counterpoise correction (CP) method, ${ }^{13}$ as implemented in Gaussian 09. All the reported dipole moments were obtained at the M06-2X/ma-def2-TZVP level in gas phase. The distribution of electrostatic potential, $V(\mathrm{r})$, on the molecular surfaces of $\mathbf{8}$ were computed at the M06-2X/TZVP level in gas phase. The $V(\mathrm{r})$ was evaluated on the $0.001 \mathrm{e} / \mathrm{Bohr}^{3}$ contour of $\rho(\mathrm{r})$ to generate the $V_{\mathrm{S}}(\mathrm{r})$. The most negative and most positive $V_{\mathrm{S}}(\mathrm{r})$ values (i.e., $V_{\mathrm{S} \text {, min }}$ and $V_{\mathrm{S} \text {,max }}$, respectively) for electrostatic potential surface energies were calculated. The QTAIM analyses ${ }^{14}$ were based on the optimized geometries of dimers in $\mathrm{CHCl}_{3}$. The estimated energies $\left(E_{\mathrm{HB}}\right)$ of hydrogen bonding interactions (for $\mathrm{X}-\mathrm{H} \cdots \mathrm{O}$ type in neutral system, $\mathrm{X}=\mathrm{C}, \mathrm{N}, \mathrm{O})$ were calculated using the empirical formula $\left[E_{\mathrm{HB}}=1 / 2 V(\mathrm{r})\right]$ reported by Espinosa et al. ${ }^{15}$ where $V(\mathrm{r})$ denotes the local potential electron energy density at the $(3,-1)$ bond critical points (BCPs). Of note, while it is clear that the estimated $E_{\mathrm{HB}}$ have important role in energetic aspect of the dimer complex, they are not the total interaction energy (Table S20). The quantitative molecular surface analysis, NCI plot and QTAIM analysis were performed by using the Multiwfn program. ${ }^{16}$

Energy decomposition calculations were undertaken on these optimized dimeric complexes using SAPT0.${ }^{17}$ Based on a rigorous perturbation expansion of the intermolecular interactions, SAPT0 energies can be decomposed into a sum of four physically meaningful contributions; i.e., electrostatic $\left(E_{\text {ele }}\right)$, dispersion $\left(E_{\text {dis }}\right)$, induction $\left(E_{\text {ind }}\right)$, and exchange repulsion $\left(E_{\text {exc }}\right)$. The first three components are attractive whereas the last is repulsive (Table S22). All of the SAPT0 calculations have been performed using the Psi4 open-source quantum chemistry package, ${ }^{18}$ employing the density fitting procedure. Considering the molecular system size, the jun-ccpVDZ basis-set (truncated Dunning-style basis set) was adopted for all the calculations since it provides the best trade-off between computational cost and accuracy. ${ }^{19}$ 


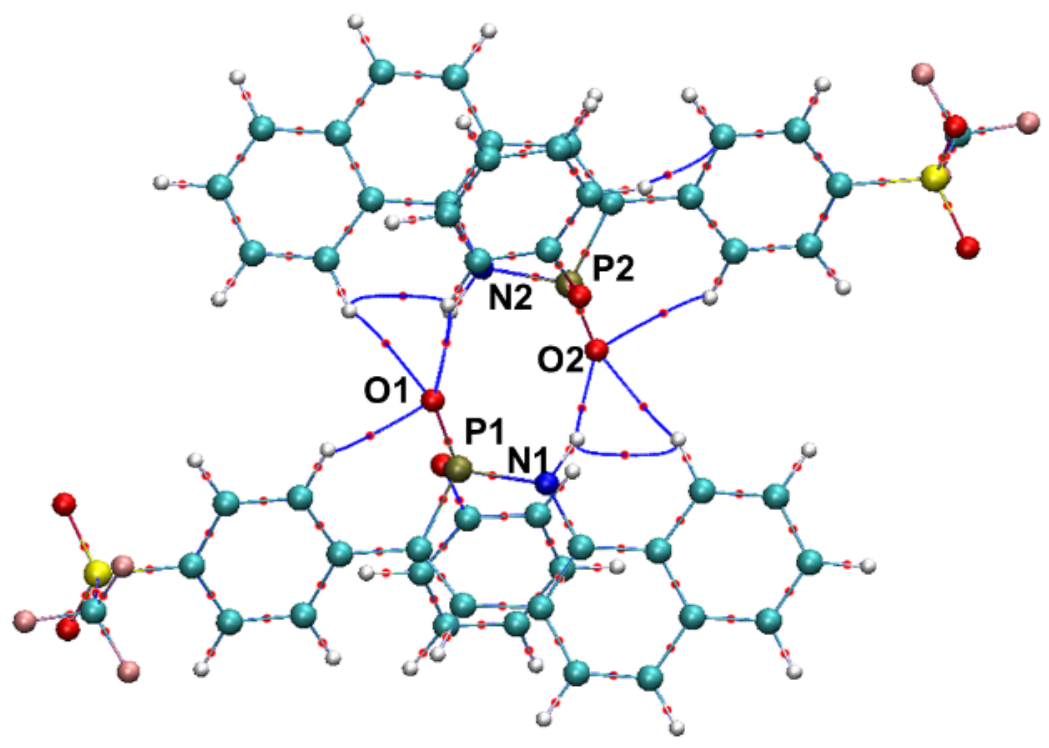

Figure S10. The representative QTAIM analysis graphs for $\mathbf{8 b} \mathbf{8 b}$ complex showing the $(3,-1)$ bond critical points (BCPs) (red dots) and bond paths (blue dotted lines).

Table S20. Double hydrogen bond lengths $d$ (in $\AA$ ), topological analysis values (in a.u.) of the density of all electrons $\rho(r)$, Laplacian of electron density $\nabla^{2} \rho(r)$ and local potential electron energy density $V(r)$ at the $(3,-1)$ bond critical points (BCPs) as well as the estimated energies $E_{\mathrm{HB}}$ (in $\mathrm{kcal} \mathrm{mol}^{-1}$ ) of these bonds among the optimized dimeric complexes, calculated at PCM( $\left(\mathrm{CHCl}_{3}\right)-\mathrm{M} 06-2 \mathrm{X} / \mathrm{TZVP}$ level of theory.

\begin{tabular}{cccccc}
\hline & $d$ & $\rho(\mathrm{r})$ & $\nabla^{2} \rho(\mathrm{r})$ & $V(\mathrm{r})$ & $E_{\mathrm{HB}}$ \\
\hline $\mathbf{8 a}$ & $1.86 ; 1.86$ & $0.0302 ; 0.0302$ & $0.1157 ; 0.1156$ & $-0.0281 ;-0.0280$ & $-8.9 ;-8.8$ \\
$\mathbf{8 b}$ & $1.85 ; 1.86$ & $0.0305 ; 0.0302$ & $0.1144 ; 0.1170$ & $-0.0285 ;-0.0279$ & $-8.9 ;-8.8$ \\
$\mathbf{8 c}$ & $1.86 ; 1.86$ & $0.0305 ; 0.0305$ & $0.1156 ; 0.1156$ & $-0.0283 ;-0.0283$ & $-8.9 ;-8.9$ \\
$\mathbf{8 d}$ & $1.86 ; 1.85$ & $0.0305 ; 0.0308$ & $0.1145 ; 0.1159$ & $-0.0285 ;-0.0286$ & $-8.9 ;-9.1$ \\
$\mathbf{8 e}$ & $1.86 ; 1.85$ & $0.0301 ; 0.0306$ & $0.1137 ; 0.1171$ & $-0.0277 ;-0.0286$ & $-8.7 ;-9.0$ \\
$\mathbf{8 f}$ & $1.84 ; 1.86$ & $0.0314 ; 0.0290$ & $0.1187 ; 0.1133$ & $-0.0299 ;-0.0271$ & $-9.4 ;-8.5$ \\
\hline
\end{tabular}




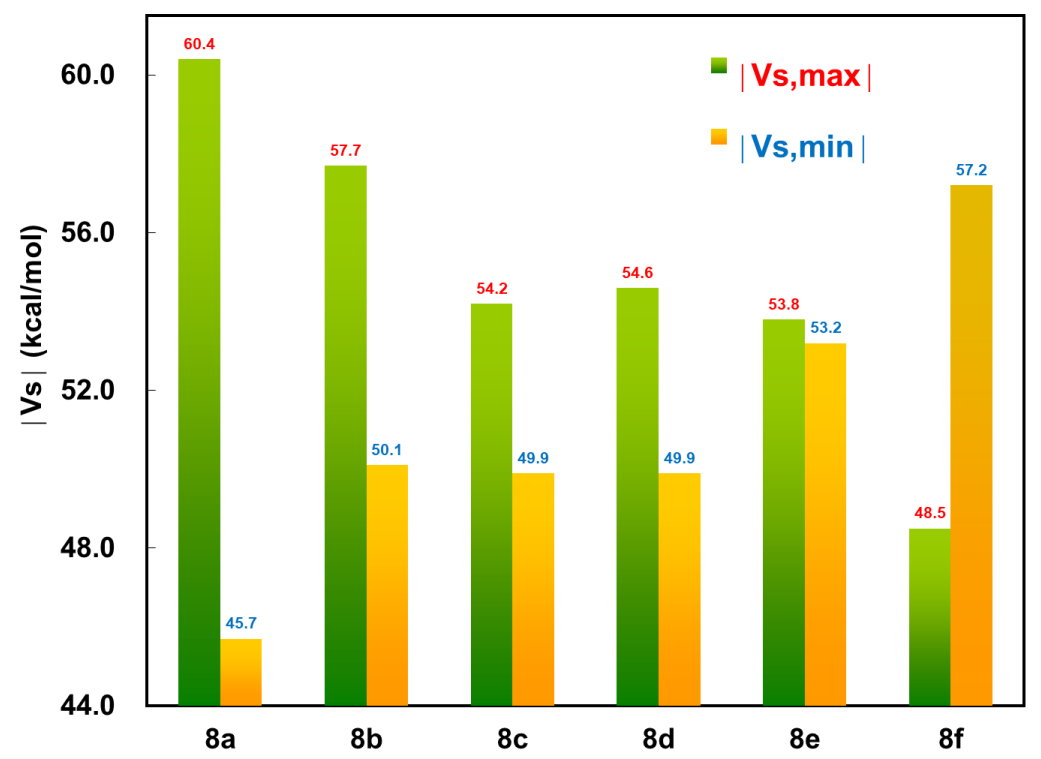

Figure S11. The calculated electrostatic potential surface minima absolute value $\left(\left|\mathrm{V}_{\mathrm{s}, \min }\right|\right)$ and maxima absolute value $\left(\left|\mathrm{V}_{\mathrm{s}, \max }\right|\right)$ of $\mathbf{8}$.

Table S21 The interaction energies $\Delta E_{\text {int }}{ }^{a}$ (in gas phase, all in a.u.), electronic energies for $\mathbf{8}$ and their dimeric complexes and BSSE energies $\left(E_{\mathrm{BSSE}}\right)$ for the intermolecular interactions calculated at M062X/ma-def2-TZVP//PCM(CHCl $)$-M06-2X/TZVP level of theory.

\begin{tabular}{ccccc}
\hline & $E_{\text {monomer }}$ & $E_{\text {dimer }}$ & $E_{\text {BSSE }}$ & $\Delta E_{\text {int }}$ \\
\hline $\mathbf{8 a}$ & -1656.496751 & -3313.041598 & 0.001113037 & -0.046982963 \\
$\mathbf{8 b}$ & -2357.697214 & -4715.442129 & 0.001108823 & -0.046592177 \\
$\mathbf{8 c}$ & -1564.253165 & -3128.553662 & 0.001090228 & -0.046241772 \\
$\mathbf{8 d}$ & -2059.937954 & -4119.923383 & 0.00110601 & -0.04636899 \\
$\mathbf{8 e}$ & -1809.097417 & -3618.241641 & 0.001043752 & -0.045763248 \\
$\mathbf{8 f}$ & -1586.530518 & -3173.107187 & 0.00096453 & -0.04518647 \\
\hline
\end{tabular}

${ }^{a} \Delta E_{\text {int }}=E_{\text {dimer }}-2 E_{\text {monomer }}+E_{\text {BSSE. }}$

Table S22 $E_{\text {ele }}, E_{\text {exc }}, E_{\text {ind }}$ and $E_{\text {dis, }}$, in the SAPT0 approach correspond to electrostatic exchange, induction and dispersion energy terms and total SAPT0 energy $\left(E_{\mathrm{SAPT}}\right)$, respectively. All values are in $\mathrm{kJ} \mathrm{mol}^{-1}$.

\begin{tabular}{cccccc}
\hline & $E_{\text {ele }}$ & $E_{\text {exc }}$ & $E_{\text {ind }}$ & $E_{\text {dis }}$ & $E_{\text {SAPT }}$ \\
\hline $\mathbf{8 a}$ & -176.4 & 143.8 & -64.0 & -73.6 & -170.2 \\
$\mathbf{8 b}$ & -166.4 & 138.5 & -61.6 & -66.7 & -156.3 \\
$\mathbf{8 c}$ & -165.3 & 141.6 & -63.1 & -68.0 & -154.8 \\
$\mathbf{8 d}$ & -164.5 & 137.4 & -60.8 & -66.9 & -154.8 \\
$\mathbf{8 e}$ & -163.3 & 139.5 & -62.4 & -66.6 & -152.8 \\
$\mathbf{8 f}$ & -162.7 & 144.8 & -65.1 & -69.3 & -152.3 \\
\hline
\end{tabular}




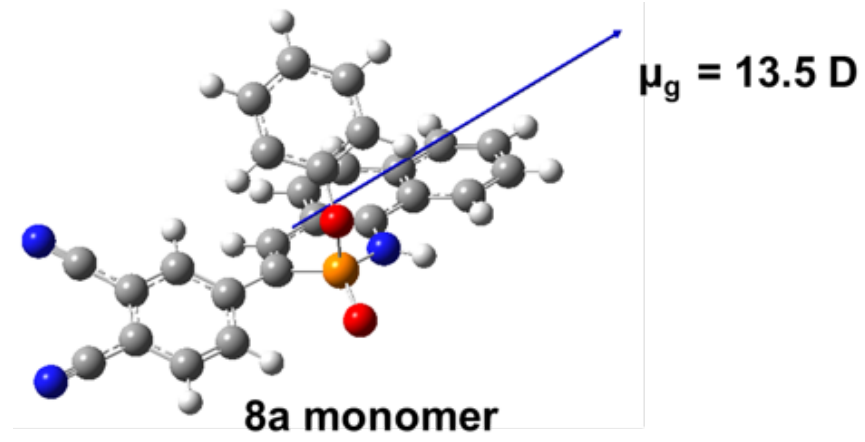

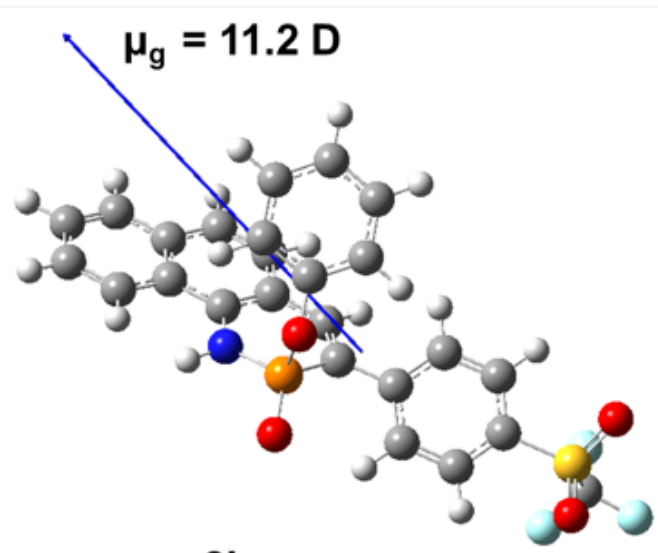

8b monomer

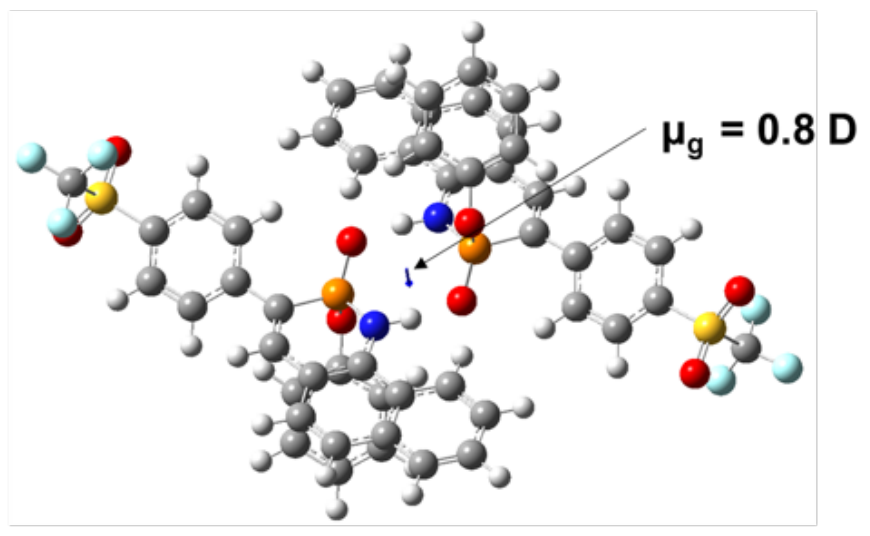

8a dimer

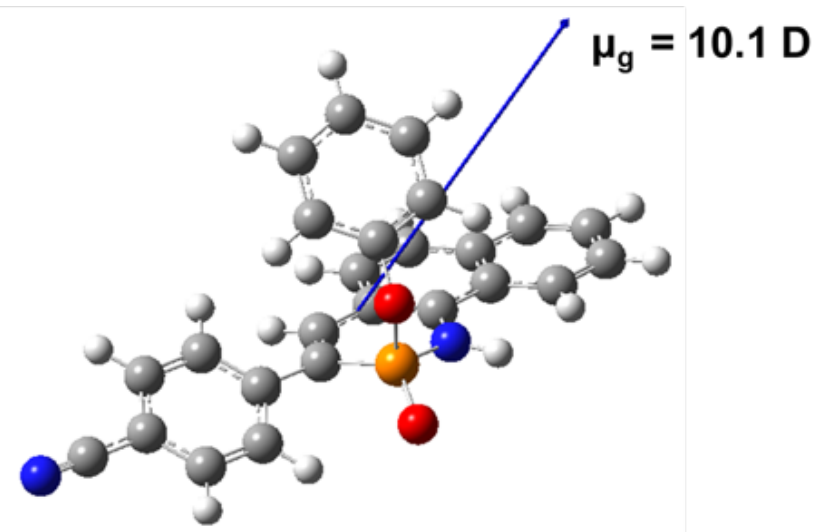

8c monomer 


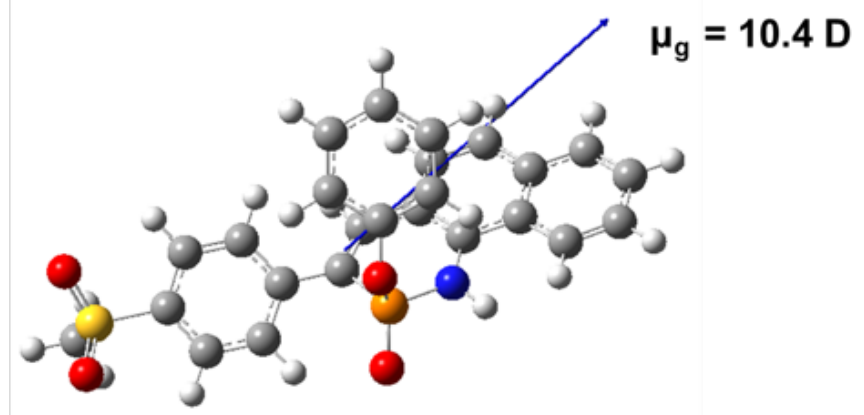

8d monomer

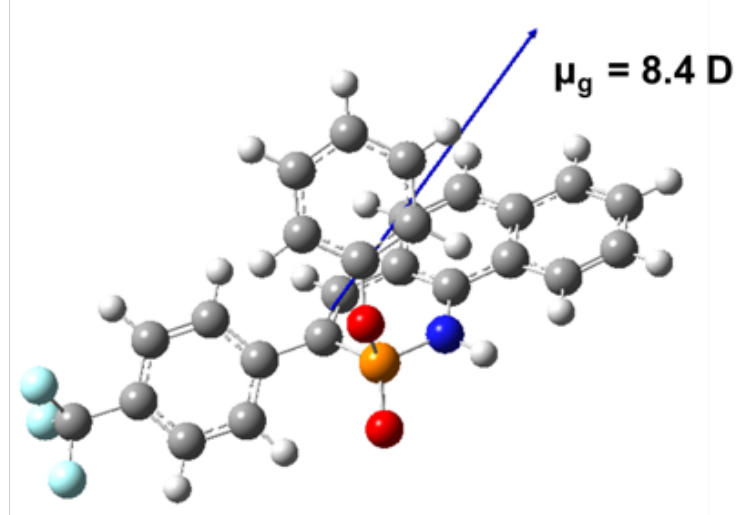

8e monomer
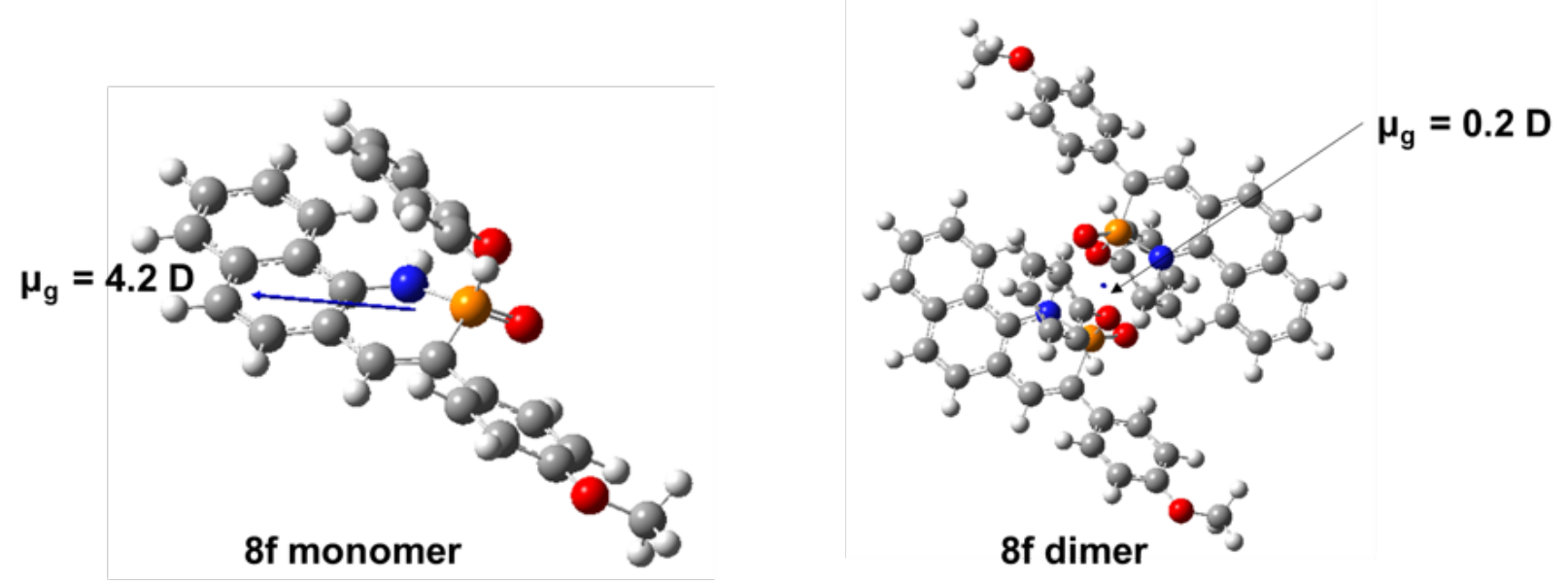

Figure S12. The plots of molecular dipole moments (in Debye) for 8 and their respective dimer with the vectors origining from the center of mass. 
Table S23. Cartesian coordinates for compund $\mathbf{8 a}$

\begin{tabular}{|c|c|c|c|}
\hline & $\mathrm{X}$ & Y & $\mathrm{Z}$ \\
\hline $\mathrm{P}$ & -0.06729 & -1.26801 & -1.30942 \\
\hline $\mathrm{O}$ & 0.04071 & 0.03486 & -2.29522 \\
\hline $\mathrm{O}$ & 0.37326 & -2.45608 & -2.08912 \\
\hline $\mathrm{N}$ & -1.66192 & -1.35598 & -0.79442 \\
\hline $\mathrm{C}$ & 0.77801 & -0.80799 & 0.19344 \\
\hline $\mathrm{C}$ & 0.04930 & -0.39275 & 1.25290 \\
\hline $\mathrm{C}$ & -1.38734 & -0.32079 & 1.35347 \\
\hline $\mathrm{C}$ & -2.21483 & -0.79972 & 0.34467 \\
\hline $\mathrm{C}$ & -3.64042 & -0.74494 & 0.48793 \\
\hline $\mathrm{C}$ & -4.17980 & -0.21533 & 1.68895 \\
\hline $\mathrm{C}$ & -3.31175 & 0.26289 & 2.70998 \\
\hline $\mathrm{C}$ & -1.96664 & 0.21059 & 2.54032 \\
\hline $\mathrm{C}$ & -4.53031 & -1.17965 & -0.52432 \\
\hline $\mathrm{C}$ & -5.88668 & -1.11523 & -0.34258 \\
\hline $\mathrm{C}$ & -6.42121 & -0.60803 & 0.85802 \\
\hline $\mathrm{C}$ & -5.58423 & -0.16573 & 1.84686 \\
\hline $\mathrm{C}$ & 2.25553 & -0.79859 & 0.20146 \\
\hline $\mathrm{C}$ & 2.93731 & 0.23159 & 0.85557 \\
\hline $\mathrm{C}$ & 4.32397 & 0.24367 & 0.89335 \\
\hline $\mathrm{C}$ & 5.05671 & -0.77144 & 0.25873 \\
\hline $\mathrm{C}$ & 4.38040 & -1.78528 & -0.41033 \\
\hline $\mathrm{C}$ & 2.99552 & -1.79588 & -0.44118 \\
\hline $\mathrm{C}$ & -0.37704 & 1.28734 & -1.86104 \\
\hline $\mathrm{C}$ & 0.56146 & 2.16183 & -1.33363 \\
\hline $\mathrm{C}$ & 0.15114 & 3.42275 & -0.92032 \\
\hline $\mathrm{C}$ & -1.18285 & 3.79729 & -1.03433 \\
\hline $\mathrm{C}$ & -2.10896 & 2.91121 & -1.57179 \\
\hline $\mathrm{C}$ & -1.70950 & 1.64847 & -1.99181 \\
\hline $\mathrm{H}$ & -2.27877 & -1.85343 & -1.42505 \\
\hline $\mathrm{H}$ & 0.58851 & -0.09133 & 2.14827 \\
\hline $\mathrm{H}$ & -3.74394 & 0.66599 & 3.61748 \\
\hline $\mathrm{H}$ & -1.29831 & 0.57316 & 3.31264 \\
\hline $\mathrm{H}$ & -4.15874 & -1.56039 & -1.46767 \\
\hline $\mathrm{H}$ & -6.55160 & -1.45112 & -1.12824 \\
\hline $\mathrm{H}$ & -7.49485 & -0.56442 & 0.99241 \\
\hline $\mathrm{H}$ & -5.98462 & 0.23525 & 2.77088 \\
\hline $\mathrm{H}$ & 2.39128 & 1.04045 & 1.32403 \\
\hline $\mathrm{H}$ & 2.48153 & -2.59432 & -0.96068 \\
\hline $\mathrm{H}$ & 1.59617 & 1.84834 & -1.26035 \\
\hline $\mathrm{H}$ & 0.87741 & 4.11474 & -0.51197 \\
\hline $\mathrm{H}$ & -1.49917 & 4.78066 & -0.71027 \\
\hline $\mathrm{H}$ & -3.14757 & 3.20181 & -1.66914 \\
\hline
\end{tabular}




$\begin{array}{rrrr}\mathrm{H} & -2.41563 & 0.94803 & -2.42041 \\ \mathrm{C} & 6.48854 & -0.76555 & 0.29339 \\ \mathrm{~N} & 7.63615 & -0.76751 & 0.31957 \\ \mathrm{C} & 4.99950 & 1.31182 & 1.57128 \\ \mathrm{~N} & 5.53116 & 2.17046 & 2.11665 \\ \mathrm{H} & 4.94310 & -2.56740 & -0.90252\end{array}$

Table S24. Cartesian coordinates for compund 8a $\bullet \mathbf{8 a}$

\begin{tabular}{|c|c|c|c|}
\hline & $X$ & $\mathrm{Y}$ & $\mathrm{Z}$ \\
\hline $\mathrm{P}$ & 1.90604 & -0.50927 & 0.28823 \\
\hline $\mathrm{O}$ & 2.14538 & -0.36271 & -1.31994 \\
\hline $\mathrm{O}$ & 1.30666 & 0.78854 & 0.74063 \\
\hline $\mathrm{N}$ & 0.94770 & -1.85024 & 0.53426 \\
\hline $\mathrm{C}$ & 3.46091 & -1.01286 & 0.99139 \\
\hline $\mathrm{C}$ & 3.62819 & -2.31092 & 1.33514 \\
\hline $\mathrm{C}$ & 2.66970 & -3.37543 & 1.22620 \\
\hline $\mathrm{C}$ & 1.36740 & -3.13848 & 0.79829 \\
\hline $\mathrm{C}$ & 0.44462 & -4.22575 & 0.64934 \\
\hline $\mathrm{C}$ & 0.87736 & -5.53104 & 0.99535 \\
\hline $\mathrm{C}$ & 2.20303 & -5.74108 & 1.47065 \\
\hline $\mathrm{C}$ & 3.06377 & -4.69977 & 1.57139 \\
\hline $\mathrm{C}$ & -0.86300 & -4.05174 & 0.13408 \\
\hline $\mathrm{C}$ & -1.70455 & -5.12350 & -0.01092 \\
\hline $\mathrm{C}$ & -1.28226 & -6.41539 & 0.35530 \\
\hline $\mathrm{C}$ & -0.02017 & -6.61137 & 0.84717 \\
\hline $\mathrm{C}$ & 4.55690 & -0.02761 & 1.06906 \\
\hline $\mathrm{C}$ & 5.87216 & -0.40844 & 0.78195 \\
\hline $\mathrm{C}$ & 6.91386 & 0.49795 & 0.85931 \\
\hline $\mathrm{C}$ & 6.66430 & 1.82290 & 1.20587 \\
\hline $\mathrm{C}$ & 5.34774 & 2.22166 & 1.47458 \\
\hline $\mathrm{C}$ & 4.30638 & 1.30429 & 1.40853 \\
\hline $\mathrm{C}$ & 2.44242 & -1.49474 & -2.07112 \\
\hline $\mathrm{C}$ & 3.76675 & -1.78475 & -2.35747 \\
\hline $\mathrm{C}$ & 4.05908 & -2.91637 & -3.10845 \\
\hline $\mathrm{C}$ & 3.03573 & -3.74204 & -3.55574 \\
\hline $\mathrm{C}$ & 1.71267 & -3.43015 & -3.26449 \\
\hline $\mathrm{C}$ & 1.40597 & -2.29791 & -2.52262 \\
\hline $\mathrm{H}$ & 4.59337 & -2.59754 & 1.74740 \\
\hline $\mathrm{H}$ & 2.50958 & -6.74483 & 1.73637 \\
\hline $\mathrm{H}$ & 4.07824 & -4.85586 & 1.91908 \\
\hline $\mathrm{H}$ & -1.21614 & -3.07252 & -0.16600 \\
\hline $\mathrm{H}$ & -2.69609 & -4.98348 & -0.42171 \\
\hline
\end{tabular}




\begin{tabular}{|c|c|c|c|}
\hline $\mathrm{H}$ & -1.95937 & -7.25171 & 0.23761 \\
\hline $\mathrm{H}$ & 0.31660 & -7.60376 & 1.12252 \\
\hline $\mathrm{H}$ & 6.07613 & -1.42542 & 0.47066 \\
\hline $\mathrm{H}$ & 3.29619 & 1.62611 & 1.62741 \\
\hline $\mathrm{H}$ & 4.54292 & -1.11949 & -1.99903 \\
\hline $\mathrm{H}$ & 5.08928 & -3.15140 & -3.34445 \\
\hline $\mathrm{H}$ & 3.26873 & -4.62563 & -4.13553 \\
\hline $\mathrm{H}$ & 0.91283 & -4.06982 & -3.61484 \\
\hline $\mathrm{H}$ & 0.38407 & -2.02557 & -2.28402 \\
\hline $\mathrm{C}$ & -7.75450 & -2.76046 & -1.28954 \\
\hline $\mathrm{N}$ & -8.63693 & -3.49291 & -1.34061 \\
\hline $\mathrm{C}$ & 7.74730 & 2.75882 & 1.27266 \\
\hline $\mathrm{N}$ & 8.62960 & 3.49152 & 1.32249 \\
\hline $\mathrm{C}$ & -5.05963 & -3.58857 & -1.82108 \\
\hline $\mathrm{N}$ & -4.79681 & -4.67632 & -2.07713 \\
\hline $\mathrm{H}$ & -7.93492 & -0.19380 & -0.65486 \\
\hline $\mathrm{H}$ & -0.02237 & -1.68990 & 0.24884 \\
\hline $\mathrm{H}$ & 7.92716 & 0.19297 & 0.63471 \\
\hline $\mathrm{C}$ & 5.05341 & 3.58538 & 1.81147 \\
\hline $\mathrm{N}$ & 4.79082 & 4.67277 & 2.06928 \\
\hline $\mathrm{P}$ & -1.91382 & 0.50661 & -0.29632 \\
\hline $\mathrm{O}$ & -2.15559 & 0.36226 & 1.31163 \\
\hline $\mathrm{O}$ & -1.31448 & -0.79207 & -0.74630 \\
\hline $\mathrm{N}$ & -0.95444 & 1.84676 & -0.54289 \\
\hline $\mathrm{C}$ & -3.46739 & 1.01004 & -1.00249 \\
\hline $\mathrm{C}$ & -3.63370 & 2.30792 & -1.34743 \\
\hline $\mathrm{C}$ & -2.67506 & 3.37220 & -1.23769 \\
\hline $\mathrm{C}$ & -1.37334 & 3.13503 & -0.80814 \\
\hline $\mathrm{C}$ & -0.45032 & 4.22204 & -0.65881 \\
\hline $\mathrm{C}$ & -0.88226 & 5.52731 & -1.00588 \\
\hline $\mathrm{C}$ & -2.20734 & 5.73758 & -1.48273 \\
\hline $\mathrm{C}$ & -3.06830 & 4.69650 & -1.58397 \\
\hline $\mathrm{C}$ & 0.85670 & 4.04784 & -0.14208 \\
\hline $\mathrm{C}$ & 1.69846 & 5.11940 & 0.00326 \\
\hline $\mathrm{C}$ & 1.27698 & 6.41126 & -0.36400 \\
\hline $\mathrm{C}$ & 0.01547 & 6.60743 & -0.85727 \\
\hline $\mathrm{C}$ & -4.56355 & 0.02507 & -1.08151 \\
\hline $\mathrm{C}$ & -5.87934 & 0.40667 & -0.79788 \\
\hline $\mathrm{C}$ & -6.92120 & -0.49941 & -0.87673 \\
\hline $\mathrm{C}$ & -6.67133 & -1.82484 & -1.22125 \\
\hline $\mathrm{C}$ & -5.35428 & -2.22438 & -1.48637 \\
\hline $\mathrm{C}$ & -4.31274 & -1.30731 & -1.41890 \\
\hline $\mathrm{C}$ & -2.45305 & 1.49537 & 2.06101 \\
\hline $\mathrm{C}$ & -3.77759 & 1.78614 & 2.34559 \\
\hline $\mathrm{C}$ & -4.07038 & 2.91879 & 3.09483 \\
\hline $\mathrm{C}$ & -3.04725 & 3.74471 & 3.54219 \\
\hline $\mathrm{C}$ & -1.72398 & 3.43204 & 3.25274 \\
\hline
\end{tabular}




$\begin{array}{lrrr}\mathrm{C} & -1.41684 & 2.29879 & 2.51263 \\ \mathrm{H} & 0.01533 & 1.68625 & -0.25668 \\ \mathrm{H} & -4.59810 & 2.59456 & -1.76148 \\ \mathrm{H} & -2.51327 & 6.74131 & -1.74924 \\ \mathrm{H} & -4.08233 & 4.85275 & -1.93289 \\ \mathrm{H} & 1.20924 & 3.06866 & 0.15881 \\ \mathrm{H} & 2.68953 & 4.97924 & 0.41511 \\ \mathrm{H} & 1.95424 & 7.24741 & -0.24600 \\ \mathrm{H} & -0.32069 & 7.59979 & -1.13345 \\ \mathrm{H} & -6.08375 & 1.42406 & -0.48823 \\ \mathrm{H} & -3.30217 & -1.62976 & -1.63504 \\ \mathrm{H} & -4.55359 & 1.12066 & 1.98718 \\ \mathrm{H} & -5.10076 & 3.15440 & 3.32947 \\ \mathrm{H} & -3.28058 & 4.62906 & 4.12066 \\ \mathrm{H} & -0.92432 & 4.07190 & 3.60317 \\ \mathrm{H} & -0.39475 & 2.02586 & 2.27549\end{array}$

Table S25. Cartesian coordinates for compund $\mathbf{8 b}$

\begin{tabular}{rrrr}
\hline & \multicolumn{1}{l}{ X } & \multicolumn{1}{l}{$\mathrm{Y}$} \\
\hline $\mathrm{S}$ & 5.61567 & 0.37629 & 0.07635 \\
$\mathrm{P}$ & -1.24911 & 0.13035 & 1.77483 \\
$\mathrm{O}$ & -1.39075 & 1.75998 & 1.68533 \\
$\mathrm{O}$ & -0.74411 & -0.20093 & 3.13403 \\
$\mathrm{O}$ & 6.18686 & 0.45726 & 1.41966 \\
$\mathrm{O}$ & 5.97790 & 1.32825 & -0.97330 \\
$\mathrm{~N}$ & -2.77131 & -0.50598 & 1.45944 \\
$\mathrm{C}$ & -0.34080 & -0.35107 & 0.31669 \\
$\mathrm{C}$ & -1.02404 & -0.81521 & -0.75230 \\
$\mathrm{C}$ & -2.44367 & -1.04598 & -0.85464 \\
$\mathrm{C}$ & -3.28853 & -0.90261 & 0.23984 \\
$\mathrm{C}$ & -4.68917 & -1.18673 & 0.11631 \\
$\mathrm{C}$ & -5.18381 & -1.61649 & -1.14283 \\
$\mathrm{C}$ & -4.29852 & -1.75536 & -2.24760 \\
$\mathrm{C}$ & -2.97824 & -1.47938 & -2.10059 \\
$\mathrm{C}$ & -5.60127 & -1.03897 & 1.18946 \\
$\mathrm{C}$ & -6.93231 & -1.31962 & 1.02565 \\
$\mathrm{C}$ & -7.41984 & -1.75753 & -0.22129 \\
$\mathrm{C}$ & -6.56258 & -1.89960 & -1.27894 \\
$\mathrm{C}$ & 1.12215 & -0.14404 & 0.28104 \\
$\mathrm{C}$ & 1.72638 & 0.33877 & -0.88721 \\
$\mathrm{C}$ & 3.09726 & 0.50483 & -0.95742 \\
$\mathrm{C}$ & 3.85790 & 0.19577 & 0.16403
\end{tabular}




$\begin{array}{rrrr}\text { C } & 3.28933 & -0.26129 & 1.34509 \\ \mathrm{C} & 1.91526 & -0.42322 & 1.40014 \\ \mathrm{C} & 6.15973 & -1.29851 & -0.57959 \\ \mathrm{C} & -1.85599 & 2.36194 & 0.52246 \\ \mathrm{C} & -0.93322 & 2.86766 & -0.38160 \\ \mathrm{C} & -1.38994 & 3.48541 & -1.53828 \\ \mathrm{C} & -2.75440 & 3.59116 & -1.78390 \\ \mathrm{C} & -3.66555 & 3.08830 & -0.86284 \\ \mathrm{C} & -3.22060 & 2.47268 & 0.30065 \\ \mathrm{H} & -3.36924 & -0.57216 & 2.27423 \\ \mathrm{H} & -0.45032 & -1.07410 & -1.63912 \\ \mathrm{H} & -4.69722 & -2.08624 & -3.19873 \\ \mathrm{H} & -2.29524 & -1.58619 & -2.93529 \\ \mathrm{H} & -5.26870 & -0.69503 & 2.16026 \\ \mathrm{H} & -7.61452 & -1.20012 & 1.85776 \\ \mathrm{H} & -8.47369 & -1.97706 & -0.33953 \\ \mathrm{H} & -6.92700 & -2.23139 & -2.24426 \\ \mathrm{H} & 1.11475 & 0.60551 & -1.74015 \\ \mathrm{H} & 3.56650 & 0.88428 & -1.85657 \\ \mathrm{H} & 3.90766 & -0.48525 & 2.20535 \\ \mathrm{H} & 1.45502 & -0.78110 & 2.31207 \\ \mathrm{H} & 0.12491 & 2.77365 & -0.16795 \\ \mathrm{H} & -0.67636 & 3.88652 & -2.24753 \\ \mathrm{H} & -3.10709 & 4.07119 & -2.68802 \\ \mathrm{H} & -4.72928 & 3.17587 & -1.04604 \\ \mathrm{H} & -3.91793 & 2.08424 & 1.03258 \\ \mathrm{~F} & 5.74680 & -2.25718 & 0.23376 \\ \mathrm{~F} & 7.47841 & -1.31545 & -0.64851 \\ \mathrm{~F} & 5.65151 & -1.49944 & -1.78513\end{array}$

Table S26. Cartesian coordinates for compund $\mathbf{8 b} \mathbf{8 b}$

\begin{tabular}{rrrr}
\hline & X & \multicolumn{1}{c}{$\mathrm{Y}$} & $\mathrm{Z}$ \\
\hline $\mathrm{S}$ & -8.59680 & -0.69151 & 0.23307 \\
$\mathrm{P}$ & -1.72217 & 1.01303 & 0.12729 \\
$\mathrm{O}$ & -1.61704 & 1.03417 & 1.75720 \\
$\mathrm{O}$ & -1.60070 & -0.42578 & -0.27372 \\
$\mathrm{O}$ & -8.45504 & -2.14314 & 0.26821 \\
$\mathrm{O}$ & -9.41169 & 0.05003 & 1.18960 \\
$\mathrm{~N}$ & -0.51945 & 2.00375 & -0.46573 \\
$\mathrm{C}$ & -3.20019 & 1.90610 & -0.29803 \\
$\mathrm{C}$ & -3.08118 & 3.20063 & -0.67082 \\
$\mathrm{C}$ & -1.86830 & 3.95303 & -0.85052
\end{tabular}




\begin{tabular}{|c|c|c|c|}
\hline $\mathrm{C}$ & -0.61881 & 3.35155 & -0.74464 \\
\hline $\mathrm{C}$ & 0.57497 & 4.11644 & -0.96265 \\
\hline $\mathrm{C}$ & 0.44736 & 5.49160 & -1.28378 \\
\hline $\mathrm{C}$ & -0.84322 & 6.08298 & -1.38953 \\
\hline $\mathrm{C}$ & -1.95416 & 5.33510 & -1.18107 \\
\hline $\mathrm{C}$ & 1.87048 & 3.55612 & -0.85117 \\
\hline $\mathrm{C}$ & 2.98326 & 4.32463 & -1.07153 \\
\hline $\mathrm{C}$ & 2.85730 & 5.68686 & -1.40280 \\
\hline $\mathrm{C}$ & 1.61603 & 6.25449 & -1.50246 \\
\hline $\mathrm{C}$ & -4.51259 & 1.24861 & -0.13579 \\
\hline $\mathrm{C}$ & -5.58857 & 1.95953 & 0.40853 \\
\hline $\mathrm{C}$ & -6.83777 & 1.37571 & 0.52427 \\
\hline $\mathrm{C}$ & -6.99562 & 0.06268 & 0.10368 \\
\hline $\mathrm{C}$ & -5.94329 & -0.67849 & -0.41525 \\
\hline $\mathrm{C}$ & -4.69906 & -0.08212 & -0.52889 \\
\hline $\mathrm{C}$ & -9.33062 & -0.30223 & -1.45320 \\
\hline $\mathrm{C}$ & -1.43163 & 2.24383 & 2.41681 \\
\hline $\mathrm{C}$ & -2.53410 & 2.90736 & 2.93084 \\
\hline $\mathrm{C}$ & -2.34355 & 4.11450 & 3.59077 \\
\hline $\mathrm{C}$ & -1.06638 & 4.64416 & 3.72480 \\
\hline $\mathrm{C}$ & 0.02772 & 3.96008 & 3.20808 \\
\hline $\mathrm{C}$ & -0.14706 & 2.74933 & 2.55251 \\
\hline $\mathrm{H}$ & 0.40849 & 1.58299 & -0.36697 \\
\hline $\mathrm{H}$ & -3.99970 & 3.74114 & -0.88939 \\
\hline $\mathrm{H}$ & -0.91759 & 7.13352 & -1.64035 \\
\hline $\mathrm{H}$ & -2.93924 & 5.77908 & -1.26487 \\
\hline $\mathrm{H}$ & 2.00689 & 2.51454 & -0.58715 \\
\hline $\mathrm{H}$ & 3.96751 & 3.88086 & -0.99181 \\
\hline $\mathrm{H}$ & 3.74482 & 6.28155 & -1.57791 \\
\hline $\mathrm{H}$ & 1.50850 & 7.30308 & -1.75339 \\
\hline $\mathrm{H}$ & -5.43582 & 2.97173 & 0.76194 \\
\hline $\mathrm{H}$ & -7.67544 & 1.91192 & 0.95209 \\
\hline $\mathrm{H}$ & -6.10267 & -1.70506 & -0.72053 \\
\hline $\mathrm{H}$ & -3.86466 & -0.63930 & -0.93435 \\
\hline $\mathrm{H}$ & -3.51768 & 2.46794 & 2.81601 \\
\hline $\mathrm{H}$ & -3.19570 & 4.64010 & 4.00256 \\
\hline $\mathrm{H}$ & -0.92292 & 5.58767 & 4.23503 \\
\hline $\mathrm{H}$ & 1.02411 & 4.36949 & 3.31484 \\
\hline $\mathrm{H}$ & 0.68630 & 2.18935 & 2.14293 \\
\hline S & 8.53361 & 0.57630 & -0.79184 \\
\hline $\mathrm{P}$ & 1.71743 & -0.99221 & 0.08287 \\
\hline $\mathrm{O}$ & 1.56776 & -1.02097 & -1.54304 \\
\hline $\mathrm{O}$ & 1.61996 & 0.45015 & 0.47884 \\
\hline $\mathrm{O}$ & 8.38563 & 2.02242 & -0.91458 \\
\hline $\mathrm{O}$ & 9.21707 & -0.24100 & -1.78870 \\
\hline $\mathrm{N}$ & 0.52225 & -1.96772 & 0.71584 \\
\hline $\mathrm{C}$ & 3.19837 & -1.89792 & 0.46867 \\
\hline
\end{tabular}




\begin{tabular}{|c|c|c|c|}
\hline $\mathrm{C}$ & 3.08145 & -3.17277 & 0.90492 \\
\hline $\mathrm{C}$ & 1.87031 & -3.90394 & 1.16299 \\
\hline $\mathrm{C}$ & 0.62174 & -3.30182 & 1.05147 \\
\hline $\mathrm{C}$ & -0.57182 & -4.05213 & 1.31635 \\
\hline $\mathrm{C}$ & -0.44445 & -5.41260 & 1.69486 \\
\hline $\mathrm{C}$ & 0.84565 & -6.00315 & 1.81171 \\
\hline $\mathrm{C}$ & 1.95609 & -5.27013 & 1.55438 \\
\hline $\mathrm{C}$ & -1.86700 & -3.49344 & 1.19263 \\
\hline $\mathrm{C}$ & -2.97981 & -4.24930 & 1.45272 \\
\hline $\mathrm{C}$ & -2.85380 & -5.59647 & 1.84089 \\
\hline $\mathrm{C}$ & -1.61303 & -6.16229 & 1.95583 \\
\hline $\mathrm{C}$ & 4.50613 & -1.27959 & 0.17065 \\
\hline $\mathrm{C}$ & 5.52107 & -2.04781 & -0.41293 \\
\hline $\mathrm{C}$ & 6.75509 & -1.49262 & -0.69960 \\
\hline $\mathrm{C}$ & 6.96107 & -0.15167 & -0.40620 \\
\hline $\mathrm{C}$ & 5.97220 & 0.64170 & 0.15760 \\
\hline $\mathrm{C}$ & 4.74208 & 0.07365 & 0.44269 \\
\hline $\mathrm{C}$ & 9.49028 & 0.30633 & 0.80318 \\
\hline $\mathrm{C}$ & 1.37390 & -2.23607 & -2.19003 \\
\hline $\mathrm{C}$ & 2.46752 & -2.89319 & -2.73074 \\
\hline $\mathrm{C}$ & 2.26918 & -4.10617 & -3.37774 \\
\hline $\mathrm{C}$ & 0.99363 & -4.64811 & -3.47154 \\
\hline $\mathrm{C}$ & -0.09160 & -3.97009 & -2.92885 \\
\hline $\mathrm{C}$ & 0.09054 & -2.75325 & -2.28652 \\
\hline $\mathrm{H}$ & -0.40619 & -1.55032 & 0.61238 \\
\hline $\mathrm{H}$ & 4.00307 & -3.71700 & 1.10005 \\
\hline $\mathrm{H}$ & 0.91974 & -7.04186 & 2.10776 \\
\hline $\mathrm{H}$ & 2.94062 & -5.71460 & 1.64215 \\
\hline $\mathrm{H}$ & -2.00367 & -2.46357 & 0.88605 \\
\hline $\mathrm{H}$ & -3.96363 & -3.80723 & 1.35958 \\
\hline $\mathrm{H}$ & -3.74110 & -6.18137 & 2.04736 \\
\hline $\mathrm{H}$ & -1.50596 & -7.19941 & 2.25072 \\
\hline $\mathrm{H}$ & 5.32628 & -3.08162 & -0.67015 \\
\hline $\mathrm{H}$ & 7.54095 & -2.07196 & -1.16759 \\
\hline $\mathrm{H}$ & 6.16812 & 1.68722 & 0.35993 \\
\hline $\mathrm{H}$ & 3.95604 & 0.67344 & 0.88291 \\
\hline $\mathrm{H}$ & 3.45000 & -2.44450 & -2.64534 \\
\hline $\mathrm{H}$ & 3.11386 & -4.62665 & -3.81092 \\
\hline $\mathrm{H}$ & 0.84426 & -5.59614 & -3.97166 \\
\hline $\mathrm{H}$ & -1.08698 & -4.38851 & -3.00624 \\
\hline $\mathrm{H}$ & -0.73528 & -2.19621 & -1.85834 \\
\hline $\mathrm{F}$ & 8.90195 & 0.95444 & 1.79704 \\
\hline $\mathrm{F}$ & 10.71961 & 0.75600 & 0.64739 \\
\hline $\mathrm{F}$ & 9.52395 & -0.98701 & 1.09040 \\
\hline $\mathrm{F}$ & -8.58444 & -0.84828 & -2.40183 \\
\hline $\mathrm{F}$ & -9.36713 & 1.00995 & -1.63403 \\
\hline $\mathrm{F}$ & -10.55340 & -0.79136 & -1.51500 \\
\hline
\end{tabular}


Table S27. Cartesian coordinates for compund 8c

\begin{tabular}{|c|c|c|c|}
\hline & $X$ & $\mathrm{Y}$ & Z \\
\hline $\mathrm{P}$ & -0.35441 & -0.70530 & 1.51094 \\
\hline $\mathrm{O}$ & -0.45461 & 0.85524 & 2.00311 \\
\hline $\mathrm{O}$ & -0.88820 & -1.55386 & 2.61019 \\
\hline $\mathrm{N}$ & 1.26338 & -1.00200 & 1.17314 \\
\hline $\mathrm{C}$ & -1.08460 & -0.74019 & -0.11643 \\
\hline $\mathrm{C}$ & -0.27001 & -0.71580 & -1.19348 \\
\hline $\mathrm{C}$ & 1.17304 & -0.72041 & -1.20652 \\
\hline $\mathrm{C}$ & 1.91358 & -0.86830 & -0.04058 \\
\hline $\mathrm{C}$ & 3.34625 & -0.90524 & -0.09016 \\
\hline $\mathrm{C}$ & 3.98219 & -0.79551 & -1.35449 \\
\hline $\mathrm{C}$ & 3.20171 & -0.64813 & -2.53457 \\
\hline $\mathrm{C}$ & 1.84744 & -0.61264 & -2.45492 \\
\hline $\mathrm{C}$ & 4.15310 & -1.02093 & 1.06821 \\
\hline $\mathrm{C}$ & 5.51952 & -1.05051 & 0.97376 \\
\hline $\mathrm{C}$ & 6.14907 & -0.95979 & -0.28320 \\
\hline $\mathrm{C}$ & 5.39446 & -0.83247 & -1.41803 \\
\hline $\mathrm{C}$ & -2.55682 & -0.68313 & -0.24047 \\
\hline $\mathrm{C}$ & -3.13999 & 0.08460 & -1.25598 \\
\hline $\mathrm{C}$ & -4.51265 & 0.13274 & -1.40832 \\
\hline $\mathrm{C}$ & -5.32718 & -0.57949 & -0.52655 \\
\hline $\mathrm{C}$ & -4.76318 & -1.33177 & 0.50295 \\
\hline $\mathrm{C}$ & -3.38715 & -1.37872 & 0.64484 \\
\hline $\mathrm{C}$ & 0.01690 & 1.87891 & 1.19068 \\
\hline $\mathrm{C}$ & -0.88371 & 2.56294 & 0.38782 \\
\hline $\mathrm{C}$ & -0.42187 & 3.59740 & -0.41533 \\
\hline $\mathrm{C}$ & 0.92626 & 3.93773 & -0.41310 \\
\hline $\mathrm{C}$ & 1.81391 & 3.24882 & 0.40442 \\
\hline $\mathrm{C}$ & 1.36261 & 2.21506 & 1.21594 \\
\hline $\mathrm{H}$ & 1.81133 & -1.28826 & 1.97537 \\
\hline $\mathrm{H}$ & -0.73802 & -0.70659 & -2.17526 \\
\hline $\mathrm{H}$ & 3.70720 & -0.56501 & -3.48876 \\
\hline $\mathrm{H}$ & 1.24517 & -0.50417 & -3.34955 \\
\hline $\mathrm{H}$ & 3.70858 & -1.07965 & 2.05386 \\
\hline $\mathrm{H}$ & 6.11882 & -1.13698 & 1.87137 \\
\hline $\mathrm{H}$ & 7.22983 & -0.98544 & -0.34621 \\
\hline $\mathrm{H}$ & 5.86799 & -0.75234 & -2.38975 \\
\hline $\mathrm{H}$ & -2.51090 & 0.66523 & -1.91956 \\
\hline $\mathrm{H}$ & -2.95137 & -1.96916 & 1.44051 \\
\hline $\mathrm{H}$ & -1.92926 & 2.27887 & 0.40256 \\
\hline
\end{tabular}




$\begin{array}{lrrr}\mathrm{H} & -1.11875 & 4.13919 & -1.04287 \\ \mathrm{H} & 1.28273 & 4.74299 & -1.04294 \\ \mathrm{H} & 2.86371 & 3.51484 & 0.41467 \\ \mathrm{H} & 2.03956 & 1.67112 & 1.86331 \\ \mathrm{C} & -6.75216 & -0.52862 & -0.67676 \\ \mathrm{~N} & -7.89370 & -0.48711 & -0.79954 \\ \mathrm{H} & -5.40153 & -1.87895 & 1.18451 \\ \mathrm{H} & -4.95869 & 0.72727 & -2.19521\end{array}$

Table S28. Cartesian coordinates for compund $\mathbf{8 c} \bullet \mathbf{8 c}$

\begin{tabular}{|c|c|c|c|}
\hline & $\mathrm{X}$ & $\mathrm{Y}$ & $\mathrm{Z}$ \\
\hline $\mathrm{P}$ & -1.91537 & -0.50274 & 0.20770 \\
\hline $\mathrm{O}$ & -2.01241 & -0.37529 & -1.41794 \\
\hline $\mathrm{O}$ & -1.37332 & 0.81255 & 0.68264 \\
\hline $\mathrm{N}$ & -0.94498 & -1.81607 & 0.54520 \\
\hline $\mathrm{C}$ & -3.51149 & -1.03924 & 0.77523 \\
\hline $\mathrm{C}$ & -3.67059 & -2.33184 & 1.13880 \\
\hline $\mathrm{C}$ & -2.66849 & -3.36367 & 1.17882 \\
\hline $\mathrm{C}$ & -1.33855 & -3.09537 & 0.87383 \\
\hline $\mathrm{C}$ & -0.35581 & -4.13949 & 0.93268 \\
\hline $\mathrm{C}$ & -0.77340 & -5.44099 & 1.30909 \\
\hline $\mathrm{C}$ & -2.13999 & -5.68879 & 1.62110 \\
\hline $\mathrm{C}$ & -3.04656 & -4.68311 & 1.55630 \\
\hline $\mathrm{C}$ & 1.00740 & -3.92170 & 0.61799 \\
\hline $\mathrm{C}$ & 1.91139 & -4.94888 & 0.68886 \\
\hline $\mathrm{C}$ & 1.49964 & -6.23939 & 1.07108 \\
\hline $\mathrm{C}$ & 0.18580 & -6.47633 & 1.37233 \\
\hline $\mathrm{C}$ & -4.63815 & -0.08468 & 0.71951 \\
\hline $\mathrm{C}$ & -5.89213 & -0.50773 & 0.26597 \\
\hline $\mathrm{C}$ & -6.96372 & 0.36501 & 0.22287 \\
\hline $\mathrm{C}$ & -6.79184 & 1.68946 & 0.62435 \\
\hline $\mathrm{C}$ & -5.54380 & 2.12831 & 1.06501 \\
\hline $\mathrm{C}$ & -4.47584 & 1.24954 & 1.10783 \\
\hline $\mathrm{C}$ & -2.25561 & -1.50744 & -2.18523 \\
\hline $\mathrm{C}$ & -3.55717 & -1.79969 & -2.56061 \\
\hline $\mathrm{C}$ & -3.79551 & -2.92653 & -3.33641 \\
\hline $\mathrm{C}$ & -2.74160 & -3.74481 & -3.72301 \\
\hline $\mathrm{C}$ & -1.44236 & -3.43114 & -3.34162 \\
\hline $\mathrm{C}$ & -1.18844 & -2.30555 & -2.56991 \\
\hline $\mathrm{H}$ & 0.03318 & -1.64608 & 0.29434 \\
\hline $\mathrm{H}$ & -4.66481 & -2.63748 & 1.45741 \\
\hline $\mathrm{H}$ & -2.43756 & -6.68911 & 1.90897 \\
\hline
\end{tabular}




\begin{tabular}{|c|c|c|c|}
\hline $\mathrm{H}$ & -4.08795 & -4.86658 & 1.79389 \\
\hline $\mathrm{H}$ & 1.36130 & -2.94441 & 0.31301 \\
\hline $\mathrm{H}$ & 2.95151 & -4.76438 & 0.45213 \\
\hline $\mathrm{H}$ & 2.22451 & -7.04159 & 1.12694 \\
\hline $\mathrm{H}$ & -0.14225 & -7.46629 & 1.66632 \\
\hline $\mathrm{H}$ & -6.01879 & -1.52749 & -0.07657 \\
\hline $\mathrm{H}$ & -3.50896 & 1.58919 & 1.45629 \\
\hline $\mathrm{H}$ & -4.35815 & -1.14009 & -2.24913 \\
\hline $\mathrm{H}$ & -4.80708 & -3.16329 & -3.64102 \\
\hline $\mathrm{H}$ & -2.93255 & -4.62366 & -4.32509 \\
\hline $\mathrm{H}$ & -0.61908 & -4.06419 & -3.64700 \\
\hline $\mathrm{H}$ & -0.18639 & -2.02951 & -2.26140 \\
\hline $\mathrm{P}$ & 1.91529 & 0.50277 & -0.20779 \\
\hline $\mathrm{O}$ & 2.01246 & 0.37526 & 1.41785 \\
\hline $\mathrm{O}$ & 1.37321 & -0.81251 & -0.68274 \\
\hline $\mathrm{N}$ & 0.94490 & 1.81611 & -0.54525 \\
\hline $\mathrm{C}$ & 3.51140 & 1.03927 & -0.77532 \\
\hline $\mathrm{C}$ & 3.67051 & 2.33188 & -1.13883 \\
\hline $\mathrm{C}$ & 2.66841 & 3.36372 & -1.17882 \\
\hline $\mathrm{C}$ & 1.33847 & 3.09542 & -0.87386 \\
\hline $\mathrm{C}$ & 0.35574 & 4.13954 & -0.93267 \\
\hline $\mathrm{C}$ & 0.77333 & 5.44105 & -1.30903 \\
\hline $\mathrm{C}$ & 2.13993 & 5.68886 & -1.62102 \\
\hline $\mathrm{C}$ & 3.04649 & 4.68317 & -1.55626 \\
\hline $\mathrm{C}$ & -1.00747 & 3.92174 & -0.61799 \\
\hline $\mathrm{C}$ & -1.91146 & 4.94892 & -0.68882 \\
\hline $\mathrm{C}$ & -1.49971 & 6.23945 & -1.07099 \\
\hline $\mathrm{C}$ & -0.18587 & 6.47639 & -1.37223 \\
\hline $\mathrm{C}$ & 4.63807 & 0.08472 & -0.71959 \\
\hline $\mathrm{C}$ & 5.89199 & 0.50774 & -0.26586 \\
\hline $\mathrm{C}$ & 6.96359 & -0.36500 & -0.22272 \\
\hline $\mathrm{C}$ & 6.79176 & -1.68942 & -0.62434 \\
\hline $\mathrm{C}$ & 5.54378 & -2.12824 & -1.06518 \\
\hline $\mathrm{C}$ & 4.47581 & -1.24947 & -1.10803 \\
\hline $\mathrm{C}$ & 2.25575 & 1.50734 & 2.18519 \\
\hline $\mathrm{C}$ & 3.55742 & 1.79978 & 2.56000 \\
\hline $\mathrm{C}$ & 3.79592 & 2.92654 & 3.33588 \\
\hline $\mathrm{C}$ & 2.74204 & 3.74455 & 3.72312 \\
\hline $\mathrm{C}$ & 1.44267 & 3.43069 & 3.34230 \\
\hline $\mathrm{C}$ & 1.18860 & 2.30519 & 2.57051 \\
\hline $\mathrm{H}$ & -0.03326 & 1.64613 & -0.29440 \\
\hline $\mathrm{H}$ & 4.66473 & 2.63753 & -1.45743 \\
\hline $\mathrm{H}$ & 2.43750 & 6.68919 & -1.90885 \\
\hline $\mathrm{H}$ & 4.08789 & 4.86665 & -1.79384 \\
\hline $\mathrm{H}$ & -1.36138 & 2.94444 & -0.31305 \\
\hline $\mathrm{H}$ & -2.95158 & 4.76441 & -0.45210 \\
\hline $\mathrm{H}$ & -2.22457 & 7.04165 & -1.12682 \\
\hline
\end{tabular}




$\begin{array}{lrrr}\mathrm{H} & 0.14219 & 7.46636 & -1.66618 \\ \mathrm{H} & 6.01860 & 1.52746 & 0.07680 \\ \mathrm{H} & 5.41908 & -3.15644 & -1.37858 \\ \mathrm{H} & 3.50899 & -1.58911 & -1.45663 \\ \mathrm{H} & 4.35839 & 1.14040 & 2.24801 \\ \mathrm{H} & 4.80759 & 3.16344 & 3.64005 \\ \mathrm{H} & 2.93310 & 4.62333 & 4.32526 \\ \mathrm{H} & 0.61942 & 4.06352 & 3.64820 \\ \mathrm{H} & 0.18645 & 2.02899 & 2.26248 \\ \mathrm{C} & -7.89825 & 2.60185 & 0.57519 \\ \mathrm{~N} & -8.78405 & 3.33183 & 0.53442 \\ \mathrm{C} & 7.89819 & -2.60179 & -0.57514 \\ \mathrm{~N} & 8.78398 & -3.33177 & -0.53433 \\ \mathrm{H} & -5.41905 & 3.15654 & 1.37830 \\ \mathrm{H} & 7.93036 & -0.03292 & 0.13201 \\ \mathrm{H} & -7.93054 & 0.03290 & -0.13171\end{array}$

Table S29. Cartesian coordinates for compund 8d

\begin{tabular}{rrrr}
\hline & \multicolumn{1}{c}{$\mathrm{X}$} & $\mathrm{Y}$ & $\mathrm{Z}$ \\
\hline $\mathrm{S}$ & -6.24291 & -0.22705 & -0.31014 \\
$\mathrm{P}$ & 0.56680 & -0.49522 & 1.64602 \\
$\mathrm{O}$ & 0.52828 & 1.10620 & 1.99079 \\
$\mathrm{O}$ & 0.06016 & -1.22155 & 2.84164 \\
$\mathrm{O}$ & -6.80348 & -0.24546 & 1.05286 \\
$\mathrm{O}$ & -6.56150 & 0.90289 & -1.20241 \\
$\mathrm{~N}$ & 2.15960 & -0.87364 & 1.27340 \\
$\mathrm{C}$ & -0.23427 & -0.66612 & 0.05980 \\
$\mathrm{C}$ & 0.53138 & -0.79272 & -1.04587 \\
$\mathrm{C}$ & 1.97163 & -0.85375 & -1.11752 \\
$\mathrm{C}$ & 2.75861 & -0.89180 & 0.02681 \\
$\mathrm{C}$ & 4.18642 & -0.97012 & -0.07513 \\
$\mathrm{C}$ & 4.77002 & -1.03818 & -1.36698 \\
$\mathrm{C}$ & 3.94264 & -1.00995 & -2.52382 \\
$\mathrm{C}$ & 2.59460 & -0.91705 & -2.39551 \\
$\mathrm{C}$ & 5.03815 & -0.96026 & 1.05651 \\
$\mathrm{C}$ & 6.39813 & -1.04101 & 0.91331 \\
$\mathrm{C}$ & 6.97556 & -1.13065 & -0.36883 \\
$\mathrm{C}$ & 6.17702 & -1.12523 & -1.48062 \\
$\mathrm{C}$ & -1.70805 & -0.55699 & -0.00999 \\
$\mathrm{C}$ & -2.30196 & 0.12978 & -1.07579 \\
$\mathrm{C}$ & -3.67910 & 0.23125 & -1.17327 \\
$\mathrm{C}$ & -4.46333 & -0.34393 & -0.18376
\end{tabular}




$\begin{array}{lrrr}\mathrm{C} & -3.90381 & -1.00813 & 0.89698 \\ \mathrm{C} & -2.52394 & -1.11183 & 0.98221 \\ \mathrm{C} & -6.73128 & -1.73582 & -1.13070 \\ \mathrm{C} & 0.96248 & 2.03510 & 1.05308 \\ \mathrm{C} & 0.01930 & 2.67220 & 0.26066 \\ \mathrm{C} & 0.44265 & 3.61583 & -0.66593 \\ \mathrm{C} & 1.79491 & 3.91190 & -0.79626 \\ \mathrm{C} & 2.72610 & 3.26980 & 0.01098 \\ \mathrm{C} & 2.31397 & 2.32790 & 0.94558 \\ \mathrm{H} & 2.73543 & -1.09264 & 2.07747 \\ \mathrm{H} & 0.02067 & -0.87139 & -2.00292 \\ \mathrm{H} & 4.40767 & -1.06372 & -3.50048 \\ \mathrm{H} & 1.95837 & -0.89432 & -3.27271 \\ \mathrm{H} & 4.63249 & -0.87431 & 2.05695 \\ \mathrm{H} & 7.03315 & -1.02993 & 1.79021 \\ \mathrm{H} & 8.05178 & -1.19657 & -0.47039 \\ \mathrm{H} & 6.61029 & -1.18509 & -2.47240 \\ \mathrm{H} & -1.67806 & 0.60994 & -1.81956 \\ \mathrm{H} & -4.14077 & 0.77248 & -1.98984 \\ \mathrm{H} & -4.53605 & -1.42943 & 1.66907 \\ \mathrm{H} & -2.07596 & -1.62912 & 1.82092 \\ \mathrm{H} & -6.43483 & -2.57860 & -0.50883 \\ \mathrm{H} & -6.24736 & -1.76865 & -2.10523 \\ \mathrm{H} & -7.81543 & -1.68983 & -1.23470 \\ \mathrm{H} & -1.02862 & 2.42453 & 0.38111 \\ \mathrm{H} & -0.28812 & 4.12129 & -1.28512 \\ \mathrm{H} & 2.12270 & 4.64670 & -1.52078 \\ \mathrm{H} & 3.77976 & 3.50212 & -0.08189 \\ \mathrm{H} & 3.02580 & 1.82534 & 1.58875\end{array}$

Table S30. Cartesian coordinates for compund $\mathbf{8 d} \bullet \mathbf{8 d}$

\begin{tabular}{llrr}
\hline & $\mathrm{X}$ & $\mathrm{Y}$ & $\mathrm{Z}$ \\
\hline $\mathrm{S}$ & -8.59831 & 0.75220 & 0.22915 \\
$\mathrm{P}$ & -1.71727 & -0.98096 & 0.04956 \\
$\mathrm{O}$ & -1.71438 & -1.01757 & -1.58386 \\
$\mathrm{O}$ & -1.56196 & 0.46058 & 0.42849 \\
$\mathrm{O}$ & -8.40339 & 2.20174 & 0.10272 \\
$\mathrm{O}$ & -9.40770 & 0.00819 & -0.74384 \\
$\mathrm{~N}$ & -0.48167 & -1.97023 & 0.57474 \\
$\mathrm{C}$ & -3.16878 & -1.86503 & 0.57155 \\
$\mathrm{C}$ & -3.02943 & -3.15364 & 0.95578 \\
$\mathrm{C}$ & -1.80859 & -3.90608 & 1.08123 \\
$\mathrm{C}$ & -0.56653 & -3.30972 & 0.89282
\end{tabular}




\begin{tabular}{|c|c|c|c|}
\hline $\mathrm{C}$ & 0.63787 & -4.07014 & 1.06634 \\
\hline $\mathrm{C}$ & 0.52742 & -5.43866 & 1.42022 \\
\hline $\mathrm{C}$ & -0.75582 & -6.02713 & 1.60296 \\
\hline $\mathrm{C}$ & -1.87643 & -5.28152 & 1.44087 \\
\hline $\mathrm{C}$ & 1.92622 & -3.51097 & 0.88439 \\
\hline $\mathrm{C}$ & 3.04962 & -4.27457 & 1.06517 \\
\hline $\mathrm{C}$ & 2.94075 & -5.63105 & 1.42530 \\
\hline $\mathrm{C}$ & 1.70646 & -6.19708 & 1.59564 \\
\hline $\mathrm{C}$ & -4.48797 & -1.20806 & 0.45935 \\
\hline $\mathrm{C}$ & -5.58792 & -1.92279 & -0.02706 \\
\hline $\mathrm{C}$ & -6.83944 & -1.33360 & -0.10288 \\
\hline $\mathrm{C}$ & -6.98155 & -0.01427 & 0.29924 \\
\hline $\mathrm{C}$ & -5.90365 & 0.72743 & 0.75763 \\
\hline $\mathrm{C}$ & -4.65572 & 0.12997 & 0.83329 \\
\hline $\mathrm{C}$ & -9.28415 & 0.43842 & 1.85657 \\
\hline $\mathrm{C}$ & -1.62296 & -2.23984 & -2.23777 \\
\hline $\mathrm{C}$ & -2.78408 & -2.85924 & -2.67198 \\
\hline $\mathrm{C}$ & -2.69036 & -4.08047 & -3.32637 \\
\hline $\mathrm{C}$ & -1.44914 & -4.66890 & -3.53434 \\
\hline $\mathrm{C}$ & -0.29523 & -4.02881 & -3.09792 \\
\hline $\mathrm{C}$ & -0.37354 & -2.80400 & -2.44979 \\
\hline $\mathrm{H}$ & 0.43913 & -1.55856 & 0.39856 \\
\hline $\mathrm{H}$ & -3.93544 & -3.69056 & 1.22869 \\
\hline $\mathrm{H}$ & -0.81669 & -7.07319 & 1.87555 \\
\hline $\mathrm{H}$ & -2.85582 & -5.72332 & 1.58353 \\
\hline $\mathrm{H}$ & 2.04924 & -2.47354 & 0.59841 \\
\hline $\mathrm{H}$ & 4.02827 & -3.83068 & 0.93283 \\
\hline $\mathrm{H}$ & 3.83660 & -6.22211 & 1.56728 \\
\hline $\mathrm{H}$ & 1.61265 & -7.24080 & 1.87150 \\
\hline $\mathrm{H}$ & -5.45195 & -2.94071 & -0.37180 \\
\hline $\mathrm{H}$ & -7.69270 & -1.87046 & -0.49891 \\
\hline $\mathrm{H}$ & -6.04375 & 1.76508 & 1.03471 \\
\hline $\mathrm{H}$ & -3.80304 & 0.69463 & 1.18772 \\
\hline $\mathrm{H}$ & -8.63737 & 0.90180 & 2.59792 \\
\hline $\mathrm{H}$ & -9.35439 & -0.63749 & 1.99847 \\
\hline $\mathrm{H}$ & -10.27129 & 0.89782 & 1.85709 \\
\hline $\mathrm{H}$ & -3.73795 & -2.37519 & -2.50020 \\
\hline $\mathrm{H}$ & -3.58996 & -4.57129 & -3.67548 \\
\hline $\mathrm{H}$ & -1.38012 & -5.62310 & -4.04035 \\
\hline $\mathrm{H}$ & 0.67361 & -4.48297 & -3.26265 \\
\hline $\mathrm{H}$ & 0.50861 & -2.27637 & -2.10525 \\
\hline S & 8.59956 & -0.78680 & -0.15702 \\
\hline $\mathrm{P}$ & 1.73333 & 0.99377 & -0.11678 \\
\hline $\mathrm{O}$ & 1.69036 & 0.99521 & 1.51674 \\
\hline $\mathrm{O}$ & 1.58386 & -0.43844 & -0.53123 \\
\hline $\mathrm{O}$ & 8.38882 & -2.23482 & -0.03758 \\
\hline $\mathrm{O}$ & 9.39939 & -0.05283 & 0.83138 \\
\hline
\end{tabular}




\begin{tabular}{|c|c|c|c|}
\hline $\mathrm{N}$ & 0.51524 & 1.99930 & -0.65172 \\
\hline $\mathrm{C}$ & 3.20131 & 1.88190 & -0.58413 \\
\hline $\mathrm{C}$ & 3.07731 & 3.17933 & -0.94221 \\
\hline $\mathrm{C}$ & 1.86260 & 3.94117 & -1.07529 \\
\hline $\mathrm{C}$ & 0.61389 & 3.34771 & -0.92690 \\
\hline $\mathrm{C}$ & -0.58255 & 4.12088 & -1.09678 \\
\hline $\mathrm{C}$ & -0.45825 & 5.49728 & -1.41361 \\
\hline $\mathrm{C}$ & 0.83139 & 6.08134 & -1.56123 \\
\hline $\mathrm{C}$ & 1.94463 & 5.32478 & -1.39838 \\
\hline $\mathrm{C}$ & -1.87689 & 3.56690 & -0.94435 \\
\hline $\mathrm{C}$ & -2.99254 & 4.34281 & -1.12026 \\
\hline $\mathrm{C}$ & -2.86990 & 5.70690 & -1.44575 \\
\hline $\mathrm{C}$ & -1.62966 & 6.26824 & -1.58546 \\
\hline $\mathrm{C}$ & 4.51299 & 1.21325 & -0.45449 \\
\hline $\mathrm{C}$ & 5.60462 & 1.90771 & 0.07726 \\
\hline $\mathrm{C}$ & 6.84922 & 1.30660 & 0.17364 \\
\hline $\mathrm{C}$ & 6.99183 & -0.00477 & -0.25328 \\
\hline $\mathrm{C}$ & 5.92115 & -0.72681 & -0.75794 \\
\hline $\mathrm{C}$ & 4.68079 & -0.11714 & -0.85525 \\
\hline $\mathrm{C}$ & 9.31563 & -0.47605 & -1.77180 \\
\hline $\mathrm{C}$ & 1.54017 & 2.19777 & 2.19645 \\
\hline $\mathrm{C}$ & 2.66643 & 2.84753 & 2.67547 \\
\hline $\mathrm{C}$ & 2.51030 & 4.04848 & 3.35542 \\
\hline $\mathrm{C}$ & 1.24332 & 4.58596 & 3.54432 \\
\hline $\mathrm{C}$ & 0.12527 & 3.91551 & 3.06212 \\
\hline $\mathrm{C}$ & 0.26547 & 2.71075 & 2.38752 \\
\hline $\mathrm{H}$ & -0.41061 & 1.58962 & -0.50263 \\
\hline $\mathrm{H}$ & 3.99179 & 3.71747 & -1.18264 \\
\hline $\mathrm{H}$ & 0.90348 & 7.13340 & -1.80649 \\
\hline $\mathrm{H}$ & 2.92884 & 5.76397 & -1.51322 \\
\hline $\mathrm{H}$ & -2.01110 & 2.52412 & -0.68368 \\
\hline $\mathrm{H}$ & -3.97573 & 3.90326 & -1.00943 \\
\hline $\mathrm{H}$ & -3.75972 & 6.30788 & -1.58402 \\
\hline $\mathrm{H}$ & -1.52489 & 7.31805 & -1.83276 \\
\hline $\mathrm{H}$ & 5.46726 & 2.91868 & 0.44135 \\
\hline $\mathrm{H}$ & 7.69555 & 1.82722 & 0.60450 \\
\hline $\mathrm{H}$ & 6.05976 & -1.75938 & -1.05436 \\
\hline $\mathrm{H}$ & 3.83403 & -0.66599 & -1.24676 \\
\hline $\mathrm{H}$ & 8.67865 & -0.93397 & -2.52492 \\
\hline $\mathrm{H}$ & 9.39599 & 0.59951 & -1.91102 \\
\hline $\mathrm{H}$ & 10.29933 & -0.94250 & -1.75571 \\
\hline $\mathrm{H}$ & 3.64162 & 2.40231 & 2.51874 \\
\hline $\mathrm{H}$ & 3.38177 & 4.56335 & 3.73943 \\
\hline $\mathrm{H}$ & 1.12675 & 5.52501 & 4.06952 \\
\hline $\mathrm{H}$ & -0.86323 & 4.33142 & 3.21016 \\
\hline $\mathrm{H}$ & -0.58639 & 2.15975 & 2.00469 \\
\hline
\end{tabular}


Table S31. Cartesian coordinates for compund 8e

\begin{tabular}{|c|c|c|c|}
\hline & $X$ & $\mathrm{Y}$ & $\mathrm{Z}$ \\
\hline $\mathrm{P}$ & -0.29534 & -0.66583 & -1.57442 \\
\hline $\mathrm{O}$ & -0.25070 & 0.90238 & -2.05197 \\
\hline $\mathrm{O}$ & 0.22224 & -1.48955 & -2.70023 \\
\hline $\mathrm{N}$ & -1.89283 & -1.00974 & -1.18922 \\
\hline $\mathrm{C}$ & 0.48779 & -0.69901 & 0.02827 \\
\hline $\mathrm{C}$ & -0.71690 & 1.90413 & -1.20968 \\
\hline $\mathrm{C}$ & -2.50843 & -0.89189 & 0.04442 \\
\hline $\mathrm{H}$ & -2.45763 & -1.30839 & -1.97511 \\
\hline $\mathrm{C}$ & -0.29256 & -0.70527 & 1.12998 \\
\hline $\mathrm{C}$ & 1.96272 & -0.60576 & 0.10461 \\
\hline $\mathrm{C}$ & 0.19545 & 2.60093 & -0.43146 \\
\hline $\mathrm{C}$ & -2.07062 & 2.20572 & -1.18053 \\
\hline $\mathrm{C}$ & -1.73508 & -0.73948 & 1.18802 \\
\hline $\mathrm{C}$ & -3.93796 & -0.94613 & 0.13540 \\
\hline $\mathrm{H}$ & 0.20620 & -0.69380 & 2.09646 \\
\hline $\mathrm{C}$ & 2.56011 & 0.16629 & 1.10942 \\
\hline $\mathrm{C}$ & 2.78072 & -1.26707 & -0.81385 \\
\hline $\mathrm{C}$ & -0.26225 & 3.61294 & 0.40208 \\
\hline $\mathrm{H}$ & 1.24655 & 2.34385 & -0.48798 \\
\hline $\mathrm{C}$ & -2.51759 & 3.21705 & -0.33903 \\
\hline $\mathrm{H}$ & -2.75676 & 1.65176 & -1.80941 \\
\hline $\mathrm{C}$ & -2.37429 & -0.65193 & 2.45643 \\
\hline $\mathrm{C}$ & -4.53864 & -0.85589 & 1.41827 \\
\hline $\mathrm{C}$ & -4.77598 & -1.05637 & -1.00106 \\
\hline $\mathrm{C}$ & 3.93601 & 0.24944 & 1.21121 \\
\hline $\mathrm{H}$ & 1.93908 & 0.72098 & 1.80212 \\
\hline $\mathrm{C}$ & 4.16266 & -1.18110 & -0.71782 \\
\hline $\mathrm{H}$ & 2.33556 & -1.86099 & -1.60169 \\
\hline $\mathrm{C}$ & -1.61775 & 3.91809 & 0.45463 \\
\hline $\mathrm{H}$ & 0.44367 & 4.16421 & 1.01099 \\
\hline $\mathrm{H}$ & -3.57338 & 3.45611 & -0.30743 \\
\hline $\mathrm{C}$ & -3.72527 & -0.70852 & 2.57569 \\
\hline $\mathrm{H}$ & -1.74795 & -0.54092 & 3.33396 \\
\hline $\mathrm{C}$ & -5.94813 & -0.90968 & 1.52164 \\
\hline $\mathrm{C}$ & -6.13869 & -1.10173 & -0.86842 \\
\hline $\mathrm{H}$ & -4.35803 & -1.09549 & -1.99912 \\
\hline $\mathrm{C}$ & 4.73516 & -0.42643 & 0.29416 \\
\hline $\mathrm{H}$ & 4.38893 & 0.84979 & 1.99160 \\
\hline $\mathrm{H}$ & 4.78511 & -1.70350 & -1.43242 \\
\hline $\mathrm{H}$ & -1.97115 & 4.70560 & 1.10819 \\
\hline
\end{tabular}




$\begin{array}{lrrr}\mathrm{H} & -4.20309 & -0.64035 & 3.54523 \\ \mathrm{C} & -6.73319 & -1.03300 & 0.40711 \\ \mathrm{H} & -6.39430 & -0.84514 & 2.50738 \\ \mathrm{H} & -6.76274 & -1.18269 & -1.74954 \\ \mathrm{H} & -7.81141 & -1.07127 & 0.50056 \\ \mathrm{C} & 6.22515 & -0.32703 & 0.43441 \\ \mathrm{~F} & 6.88031 & -0.89248 & -0.58589 \\ \mathrm{~F} & 6.66625 & -0.92462 & 1.55737 \\ \mathrm{~F} & 6.64057 & 0.94969 & 0.50642\end{array}$

Table S32. Cartesian coordinates for compund 8e•8e

\begin{tabular}{|c|c|c|c|}
\hline & $X$ & $\mathrm{Y}$ & $\mathrm{Z}$ \\
\hline $\mathrm{P}$ & -1.78816 & -0.87952 & -0.00718 \\
\hline $\mathrm{O}$ & -1.92584 & -0.95876 & -1.63350 \\
\hline $\mathrm{O}$ & -1.54531 & 0.56338 & 0.31758 \\
\hline $\mathrm{N}$ & -0.55054 & -1.90801 & 0.43154 \\
\hline $\mathrm{C}$ & -3.22182 & -1.68192 & 0.67048 \\
\hline $\mathrm{C}$ & -1.90096 & -2.20091 & -2.25429 \\
\hline $\mathrm{C}$ & -0.65838 & -3.21856 & 0.84820 \\
\hline $\mathrm{H}$ & 0.36993 & -1.54183 & 0.17123 \\
\hline $\mathrm{C}$ & -3.09361 & -2.94157 & 1.14274 \\
\hline $\mathrm{C}$ & -4.52796 & -0.99219 & 0.59844 \\
\hline $\mathrm{C}$ & -3.09799 & -2.84120 & -2.53274 \\
\hline $\mathrm{C}$ & -0.67799 & -2.76539 & -2.58546 \\
\hline $\mathrm{C}$ & -1.89537 & -3.73658 & 1.21413 \\
\hline $\mathrm{C}$ & 0.52138 & -4.03089 & 0.93178 \\
\hline $\mathrm{H}$ & -3.99143 & -3.41848 & 1.52985 \\
\hline $\mathrm{C}$ & -5.67741 & -1.70863 & 0.24627 \\
\hline $\mathrm{C}$ & -4.64412 & 0.37190 & 0.87645 \\
\hline $\mathrm{C}$ & -3.06686 & -4.08390 & -3.15206 \\
\hline $\mathrm{H}$ & -4.03020 & -2.35623 & -2.26920 \\
\hline $\mathrm{C}$ & -0.66155 & -4.01052 & -3.19809 \\
\hline $\mathrm{H}$ & 0.23230 & -2.22377 & -2.35399 \\
\hline $\mathrm{C}$ & -1.97988 & -5.07445 & 1.69141 \\
\hline $\mathrm{C}$ & 0.39432 & -5.36120 & 1.40567 \\
\hline $\mathrm{C}$ & 1.79905 & -3.56121 & 0.54233 \\
\hline $\mathrm{C}$ & -6.91279 & -1.08858 & 0.20257 \\
\hline $\mathrm{H}$ & -5.59397 & -2.75689 & -0.01372 \\
\hline $\mathrm{C}$ & -5.88189 & 0.99592 & 0.83043 \\
\hline $\mathrm{H}$ & -3.76113 & 0.93888 & 1.14175 \\
\hline $\mathrm{C}$ & -1.85166 & -4.67164 & -3.47886 \\
\hline $\mathrm{H}$ & -3.99498 & -4.59276 & -3.37923 \\
\hline
\end{tabular}




\begin{tabular}{|c|c|c|c|}
\hline $\mathrm{H}$ & 0.28626 & -4.46548 & -3.45580 \\
\hline $\mathrm{C}$ & -0.88126 & -5.86297 & 1.78819 \\
\hline $\mathrm{H}$ & -2.95401 & -5.45157 & 1.98010 \\
\hline $\mathrm{C}$ & 1.54929 & -6.17101 & 1.49001 \\
\hline $\mathrm{C}$ & 2.89818 & -4.37375 & 0.63629 \\
\hline $\mathrm{H}$ & 1.93245 & -2.55549 & 0.16281 \\
\hline $\mathrm{C}$ & -7.01253 & 0.26649 & 0.49552 \\
\hline $\mathrm{H}$ & -7.79674 & -1.64913 & -0.07556 \\
\hline $\mathrm{H}$ & -5.96565 & 2.05079 & 1.05594 \\
\hline $\mathrm{H}$ & -1.83110 & -5.64238 & -3.95691 \\
\hline $\mathrm{H}$ & -0.95578 & -6.87981 & 2.15226 \\
\hline $\mathrm{C}$ & 2.77519 & -5.69138 & 1.11582 \\
\hline $\mathrm{H}$ & 1.44285 & -7.18471 & 1.85782 \\
\hline $\mathrm{H}$ & 3.86907 & -3.99813 & 0.33964 \\
\hline $\mathrm{H}$ & 3.65194 & -6.32285 & 1.18496 \\
\hline $\mathrm{O}$ & 1.54529 & -0.47282 & -0.79240 \\
\hline $\mathrm{P}$ & 1.77738 & 0.91986 & -0.28916 \\
\hline $\mathrm{O}$ & 1.75263 & 0.81025 & 1.34202 \\
\hline $\mathrm{N}$ & 0.60281 & 2.01661 & -0.73475 \\
\hline $\mathrm{C}$ & 3.28128 & 1.76563 & -0.71596 \\
\hline $\mathrm{C}$ & 1.70292 & 1.96721 & 2.10846 \\
\hline $\mathrm{C}$ & 0.76475 & 3.36729 & -0.96135 \\
\hline $\mathrm{H}$ & -0.33948 & 1.64672 & -0.58303 \\
\hline $\mathrm{C}$ & 3.21609 & 3.07884 & -1.02771 \\
\hline $\mathrm{C}$ & 4.56258 & 1.03970 & -0.58425 \\
\hline $\mathrm{C}$ & 2.88193 & 2.48937 & 2.61663 \\
\hline $\mathrm{C}$ & 0.47488 & 2.56148 & 2.35876 \\
\hline $\mathrm{C}$ & 2.03806 & 3.90266 & -1.12037 \\
\hline $\mathrm{C}$ & -0.39375 & 4.20717 & -1.06327 \\
\hline $\mathrm{H}$ & 4.15481 & 3.58297 & -1.24742 \\
\hline $\mathrm{C}$ & 5.66545 & 1.66995 & 0.00350 \\
\hline $\mathrm{C}$ & 4.69644 & -0.27697 & -1.02971 \\
\hline $\mathrm{C}$ & 2.82813 & 3.64235 & 3.38904 \\
\hline $\mathrm{H}$ & 3.81713 & 1.98375 & 2.40841 \\
\hline $\mathrm{C}$ & 0.43706 & 3.71722 & 3.12689 \\
\hline $\mathrm{H}$ & -0.42191 & 2.10853 & 1.95120 \\
\hline $\mathrm{C}$ & 2.18182 & 5.29105 & -1.39703 \\
\hline $\mathrm{C}$ & -0.20827 & 5.58614 & -1.33553 \\
\hline $\mathrm{C}$ & -1.70947 & 3.71457 & -0.88719 \\
\hline $\mathrm{C}$ & 6.87539 & 1.01167 & 0.12167 \\
\hline $\mathrm{H}$ & 5.56031 & 2.67636 & 0.39014 \\
\hline $\mathrm{C}$ & 5.90970 & -0.93949 & -0.91054 \\
\hline $\mathrm{H}$ & 3.84765 & -0.77521 & -1.48034 \\
\hline $\mathrm{C}$ & 1.60937 & 4.25995 & 3.64011 \\
\hline $\mathrm{H}$ & 3.74161 & 4.05669 & 3.79663 \\
\hline $\mathrm{H}$ & -0.51454 & 4.19413 & 3.32422 \\
\hline $\mathrm{C}$ & 1.10503 & 6.10851 & -1.50184 \\
\hline
\end{tabular}




$\begin{array}{lrrr}\mathrm{H} & 3.18369 & 5.68487 & -1.52249 \\ \mathrm{C} & -1.34271 & 6.42239 & -1.43779 \\ \mathrm{C} & -2.78764 & 4.55322 & -0.99405 \\ \mathrm{H} & -1.89020 & 2.67100 & -0.65985 \\ \mathrm{C} & 6.99494 & -0.29551 & -0.33733 \\ \mathrm{H} & 7.72348 & 1.50323 & 0.58274 \\ \mathrm{H} & 6.01052 & -1.95515 & -1.26886 \\ \mathrm{H} & 1.57303 & 5.16038 & 4.23948 \\ \mathrm{H} & 1.22588 & 7.16382 & -1.71119 \\ \mathrm{C} & -2.60546 & 5.92080 & -1.27359 \\ \mathrm{H} & -1.19044 & 7.47420 & -1.64945 \\ \mathrm{H} & -3.78745 & 4.15900 & -0.86388 \\ \mathrm{H} & -3.46575 & 6.57296 & -1.35560 \\ \mathrm{C} & 8.32131 & -0.98629 & -0.19395 \\ \mathrm{C} & -8.36764 & 0.91420 & 0.47175 \\ \mathrm{~F} & 8.28253 & -2.25576 & -0.61293 \\ \mathrm{~F} & 8.73544 & -1.00216 & 1.08231 \\ \mathrm{~F} & 9.28242 & -0.36648 & -0.89674 \\ \mathrm{~F} & -8.29235 & 2.24990 & 0.47904 \\ \mathrm{~F} & -9.10730 & 0.55732 & 1.53402 \\ \mathrm{~F} & -9.06852 & 0.56009 & -0.61551\end{array}$

Table S33. Cartesian coordinates for compund $\mathbf{8 f}$

\begin{tabular}{lrrr}
\hline & \multicolumn{1}{l}{ X } & \multicolumn{1}{c}{$\mathrm{Y}$} & \multicolumn{1}{c}{$\mathrm{Z}$} \\
\hline $\mathrm{P}$ & -0.26940 & -0.71877 & 1.45205 \\
$\mathrm{O}$ & -0.35916 & 0.83047 & 1.98780 \\
$\mathrm{O}$ & -0.83098 & -1.58669 & 2.52326 \\
$\mathrm{~N}$ & 1.35212 & -1.03143 & 1.14550 \\
$\mathrm{C}$ & -0.96705 & -0.69813 & -0.18939 \\
$\mathrm{C}$ & 0.13426 & 1.87046 & 1.21184 \\
$\mathrm{C}$ & 2.03323 & -0.88020 & -0.05032 \\
$\mathrm{H}$ & 1.87742 & -1.33997 & 1.95388 \\
$\mathrm{C}$ & -0.12539 & -0.66316 & -1.24429 \\
$\mathrm{C}$ & -2.43475 & -0.59317 & -0.33383 \\
$\mathrm{C}$ & -0.74792 & 2.59204 & 0.42100 \\
$\mathrm{C}$ & 1.48415 & 2.18752 & 1.26177 \\
$\mathrm{C}$ & 1.31999 & -0.69098 & -1.22606 \\
$\mathrm{C}$ & 3.46570 & -0.93993 & -0.06934 \\
$\mathrm{H}$ & -0.56977 & -0.61503 & -2.23563 \\
$\mathrm{C}$ & -2.98760 & 0.20656 & -1.34512 \\
$\mathrm{C}$ & -3.30757 & -1.26834 & 0.51787 \\
$\mathrm{C}$ & -0.26308 & 3.64537 & -0.34304
\end{tabular}




$\begin{array}{lrrr}\mathrm{H} & -1.79679 & 2.32005 & 0.41335 \\ \mathrm{C} & 1.95855 & 3.24066 & 0.48947 \\ \mathrm{H} & 2.14608 & 1.61317 & 1.89830 \\ \mathrm{C} & 2.02325 & -0.56136 & -2.45520 \\ \mathrm{C} & 4.13123 & -0.80942 & -1.31674 \\ \mathrm{C} & 4.24644 & -1.10233 & 1.10186 \\ \mathrm{C} & -4.35310 & 0.30025 & -1.51322 \\ \mathrm{H} & -2.33421 & 0.77793 & -1.99435 \\ \mathrm{C} & -4.68640 & -1.17871 & 0.36077 \\ \mathrm{H} & -2.91008 & -1.88855 & 1.31172 \\ \mathrm{C} & 1.08919 & 3.96786 & -0.31463 \\ \mathrm{H} & -0.94513 & 4.21574 & -0.96159 \\ \mathrm{H} & 3.01171 & 3.49146 & 0.51912 \\ \mathrm{C} & 3.37897 & -0.61753 & -2.50771 \\ \mathrm{H} & 1.44297 & -0.41719 & -3.35941 \\ \mathrm{C} & 5.54419 & -0.86909 & -1.35156 \\ \mathrm{C} & 5.61395 & -1.15314 & 1.03495 \\ \mathrm{H} & 3.78129 & -1.18115 & 2.07669 \\ \mathrm{C} & -5.21480 & -0.39403 & -0.65983 \\ \mathrm{H} & -4.77998 & 0.92272 & -2.28978 \\ \mathrm{H} & -5.32911 & -1.72479 & 1.03706 \\ \mathrm{H} & 1.46321 & 4.78800 & -0.91451 \\ \mathrm{H} & 3.90562 & -0.51823 & -3.44891 \\ \mathrm{C} & 6.27246 & -1.03930 & -0.20542 \\ \mathrm{H} & 6.03917 & -0.77170 & -2.31106 \\ \mathrm{H} & 6.19180 & -1.27626 & 1.94234 \\ \mathrm{O} & -6.53917 & -0.23603 & -0.89646 \\ \mathrm{H} & 7.35378 & -1.08197 & -0.24625 \\ \mathrm{C} & -7.44871 & -0.91044 & -0.04154 \\ \mathrm{H} & -7.33093 & -0.58348 & 0.99476 \\ \mathrm{H} & -8.44329 & -0.64600 & -0.39254 \\ \mathrm{H} & -7.31805 & -1.99394 & -0.10309\end{array}$

Table S34. Cartesian coordinates for compund $\mathbf{8 f} \bullet \mathbf{8 f}$

\begin{tabular}{rrrr}
\hline & X & Y & Z \\
\hline P & -1.87727 & -0.68069 & 0.17148 \\
O & -1.98103 & -0.61162 & -1.45804 \\
O & -1.50782 & 0.70686 & 0.60703 \\
N & -0.74026 & -1.84587 & 0.53838 \\
C & -3.39095 & -1.40305 & 0.75905 \\
C & -2.08540 & -1.78249 & -2.19450 \\
C & -0.95502 & -3.13845 & 0.96551
\end{tabular}




\begin{tabular}{|c|c|c|c|}
\hline $\mathrm{H}$ & 0.20124 & -1.56967 & 0.24522 \\
\hline $\mathrm{C}$ & -3.36759 & -2.67436 & 1.21609 \\
\hline $\mathrm{C}$ & -4.64297 & -0.63213 & 0.60775 \\
\hline $\mathrm{C}$ & -3.34204 & -2.24345 & -2.55451 \\
\hline $\mathrm{C}$ & -0.92840 & -2.44885 & -2.57087 \\
\hline $\mathrm{C}$ & -2.23241 & -3.55678 & 1.31713 \\
\hline $\mathrm{C}$ & 0.15798 & -4.03886 & 1.06878 \\
\hline $\mathrm{H}$ & -4.31300 & -3.09374 & 1.55328 \\
\hline $\mathrm{C}$ & -5.81639 & -1.27348 & 0.18693 \\
\hline $\mathrm{C}$ & -4.69986 & 0.73630 & 0.86191 \\
\hline $\mathrm{C}$ & -3.44048 & -3.40737 & -3.30573 \\
\hline $\mathrm{H}$ & -4.21791 & -1.68415 & -2.24801 \\
\hline $\mathrm{C}$ & -1.04282 & -3.61309 & -3.31801 \\
\hline $\mathrm{H}$ & 0.03185 & -2.04233 & -2.27379 \\
\hline $\mathrm{C}$ & -2.42634 & -4.88572 & 1.78604 \\
\hline $\mathrm{C}$ & -0.07876 & -5.35824 & 1.53134 \\
\hline $\mathrm{C}$ & 1.47704 & -3.66466 & 0.71447 \\
\hline $\mathrm{C}$ & -7.00063 & -0.58091 & 0.05234 \\
\hline $\mathrm{H}$ & -5.78720 & -2.32873 & -0.05897 \\
\hline $\mathrm{C}$ & -5.88817 & 1.44598 & 0.72771 \\
\hline $\mathrm{H}$ & -3.80488 & 1.25493 & 1.18317 \\
\hline $\mathrm{C}$ & -2.29413 & -4.09425 & -3.68511 \\
\hline $\mathrm{H}$ & -4.41596 & -3.77540 & -3.59750 \\
\hline $\mathrm{H}$ & -0.14829 & -4.14564 & -3.61504 \\
\hline $\mathrm{C}$ & -1.39483 & -5.76010 & 1.89195 \\
\hline $\mathrm{H}$ & -3.43110 & -5.18702 & 2.05896 \\
\hline $\mathrm{C}$ & 1.00896 & -6.25507 & 1.63042 \\
\hline $\mathrm{C}$ & 2.50896 & -4.55941 & 0.82342 \\
\hline $\mathrm{H}$ & 1.69660 & -2.66784 & 0.35142 \\
\hline $\mathrm{C}$ & -7.04515 & 0.78778 & 0.32361 \\
\hline $\mathrm{H}$ & -7.90704 & -1.07073 & -0.27872 \\
\hline $\mathrm{H}$ & -5.89466 & 2.50472 & 0.94482 \\
\hline $\mathrm{H}$ & -2.37469 & -5.00255 & -4.26829 \\
\hline $\mathrm{H}$ & -1.55440 & -6.76994 & 2.24839 \\
\hline $\mathrm{C}$ & 2.27553 & -5.86856 & 1.28525 \\
\hline $\mathrm{H}$ & 0.81762 & -7.26021 & 1.98771 \\
\hline $\mathrm{H}$ & 3.51192 & -4.25261 & 0.55502 \\
\hline $\mathrm{O}$ & -8.25176 & 1.38434 & 0.15791 \\
\hline $\mathrm{H}$ & 3.09912 & -6.56655 & 1.36828 \\
\hline $\mathrm{C}$ & -8.33895 & 2.77199 & 0.40320 \\
\hline $\mathrm{H}$ & -7.67903 & 3.33435 & -0.26349 \\
\hline $\mathrm{H}$ & -9.37122 & 3.04863 & 0.20621 \\
\hline $\mathrm{H}$ & -8.09188 & 3.00704 & 1.44226 \\
\hline $\mathrm{O}$ & 1.41633 & -0.62147 & -0.76687 \\
\hline $\mathrm{P}$ & 1.83250 & 0.72616 & -0.25684 \\
\hline $\mathrm{O}$ & 1.92694 & 0.56219 & 1.36857 \\
\hline $\mathrm{N}$ & 0.73934 & 1.94851 & -0.56176 \\
\hline
\end{tabular}




\begin{tabular}{|c|c|c|c|}
\hline $\mathrm{C}$ & 3.37461 & 1.41953 & -0.80087 \\
\hline $\mathrm{C}$ & 2.06131 & 1.69004 & 2.16545 \\
\hline $\mathrm{C}$ & 1.01695 & 3.27206 & -0.83554 \\
\hline $\mathrm{H}$ & -0.21903 & 1.67879 & -0.32081 \\
\hline $\mathrm{C}$ & 3.41037 & 2.72834 & -1.13109 \\
\hline $\mathrm{C}$ & 4.58217 & 0.56891 & -0.75052 \\
\hline $\mathrm{C}$ & 3.32940 & 2.09980 & 2.54636 \\
\hline $\mathrm{C}$ & 0.92208 & 2.36797 & 2.57418 \\
\hline $\mathrm{C}$ & 2.31559 & 3.66719 & -1.13384 \\
\hline $\mathrm{C}$ & -0.05108 & 4.23031 & -0.83733 \\
\hline $\mathrm{H}$ & 4.37195 & 3.13392 & -1.43835 \\
\hline $\mathrm{C}$ & 5.79213 & 1.08740 & -0.26875 \\
\hline $\mathrm{C}$ & 4.55868 & -0.75917 & -1.17042 \\
\hline $\mathrm{C}$ & 3.45816 & 3.22070 & 3.35585 \\
\hline $\mathrm{H}$ & 4.19021 & 1.53339 & 2.21153 \\
\hline $\mathrm{C}$ & 1.06647 & 3.48818 & 3.38088 \\
\hline $\mathrm{H}$ & -0.04872 & 2.00549 & 2.25485 \\
\hline $\mathrm{C}$ & 2.57281 & 5.02733 & -1.46077 \\
\hline $\mathrm{C}$ & 0.24793 & 5.57818 & -1.16178 \\
\hline $\mathrm{C}$ & -1.38498 & 3.88475 & -0.51009 \\
\hline $\mathrm{C}$ & 6.93494 & 0.31648 & -0.23204 \\
\hline $\mathrm{H}$ & 5.82528 & 2.10639 & 0.09937 \\
\hline $\mathrm{C}$ & 5.70451 & -1.54638 & -1.13737 \\
\hline $\mathrm{H}$ & 3.63414 & -1.18235 & -1.54318 \\
\hline $\mathrm{C}$ & 2.32997 & 3.91692 & 3.77086 \\
\hline $\mathrm{H}$ & 4.44268 & 3.54834 & 3.66473 \\
\hline $\mathrm{H}$ & 0.18603 & 4.02831 & 3.70516 \\
\hline $\mathrm{C}$ & 1.58253 & 5.95435 & -1.47826 \\
\hline $\mathrm{H}$ & 3.59232 & 5.30883 & -1.69797 \\
\hline $\mathrm{C}$ & -0.79648 & 6.53029 & -1.16379 \\
\hline $\mathrm{C}$ & -2.37270 & 4.83427 & -0.51933 \\
\hline $\mathrm{H}$ & -1.64863 & 2.86871 & -0.24170 \\
\hline $\mathrm{C}$ & 6.89906 & -1.00890 & -0.66847 \\
\hline $\mathrm{H}$ & 7.86985 & 0.70973 & 0.14553 \\
\hline $\mathrm{H}$ & 5.64916 & -2.56850 & -1.48411 \\
\hline $\mathrm{H}$ & 2.43399 & 4.79136 & 4.40023 \\
\hline $\mathrm{H}$ & 1.79051 & 6.98741 & -1.72745 \\
\hline $\mathrm{C}$ & -2.07910 & 6.17066 & -0.85116 \\
\hline $\mathrm{H}$ & -0.55796 & 7.55674 & -1.41735 \\
\hline $\mathrm{H}$ & -3.38784 & 4.55224 & -0.26999 \\
\hline $\mathrm{O}$ & 8.06972 & -1.68941 & -0.59121 \\
\hline $\mathrm{H}$ & -2.86938 & 6.91083 & -0.85696 \\
\hline $\mathrm{C}$ & 8.07363 & -3.03908 & -1.00557 \\
\hline $\mathrm{H}$ & 9.08951 & -3.39683 & -0.86157 \\
\hline $\mathrm{H}$ & 7.38820 & -3.64032 & -0.40134 \\
\hline $\mathrm{H}$ & 7.80253 & -3.13028 & -2.06112 \\
\hline
\end{tabular}




\section{Measurements of Dimerization Constants $\boldsymbol{K}_{\mathrm{dim}}$}

Self-dimerization equilibria were studied by virtue of ${ }^{1} \mathrm{H}$ NMR spectroscopic (additional ${ }^{31} \mathrm{P}$ NMR analysis for $8 \mathbf{b}$ ) dilution experiments in water-saturated $\mathrm{CDCl}_{3}$ ( $\mathrm{ca} .0 .8 \%$ water in $\mathrm{CHCl}_{3}$ ). Nonlinear regression analysis has been performed to fit the following equation ${ }^{20}$ by using the curve-fitting technique with OriginPro 8.0 program. The error of $K_{\text {dim }}$ is the standard deviation of three measurements.

$$
\delta_{\text {obs }}=\delta_{\mathrm{m}}+\left(\delta_{\mathrm{d}}-\delta_{\mathrm{m}}\right) \frac{1+4 K_{\operatorname{dim}}[\mathrm{H}]-\sqrt{1+8 K_{\operatorname{dim}}[\mathrm{H}]}}{4 K_{\operatorname{dim}}[\mathrm{H}]}
$$

where

$[\mathrm{H}]$ is the total concentration

$K_{\text {dim is }}$ the dimerization constant

$\delta_{\mathrm{m}}$ is the free chemical shift of the monomer

$\delta_{\mathrm{d}}$ is the limiting bound chemical shift of the dimer

$\delta_{\text {obs }}$ is the observable chemical shift;

The chemical shifts at each concentration were recorded and fitted into the dimerization equation to determine the optimum solutions for the dimerization constant $\left(K_{\mathrm{dim}}\right)$, and the bound $\left(\delta_{\mathrm{d}}\right)$ and free chemical shifts $\left(\delta_{\mathrm{m}}\right)$. 

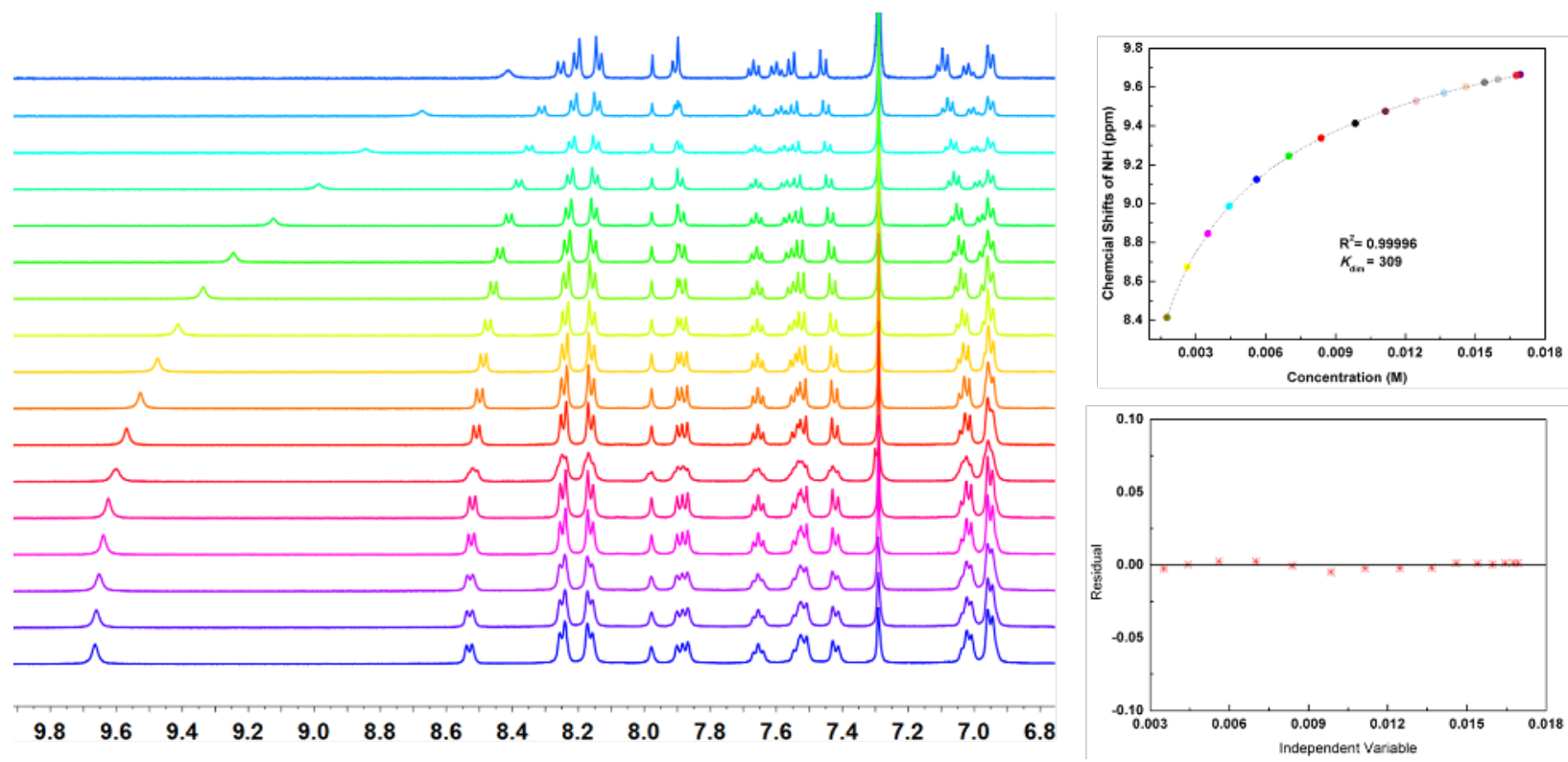

Figure S13. Stacked partial ${ }^{1} \mathrm{H}$ NMR spectra of compound $\mathbf{8 b}$ at different concentrations in water-saturated $\mathrm{CDCl}_{3}(298 \mathrm{~K})$. Determination of the dimerization constant of $\mathbf{8 b} \cdot \mathbf{8 b}$, fitting result based on chemical shifts of phosphonamidate $\mathrm{NH}$.
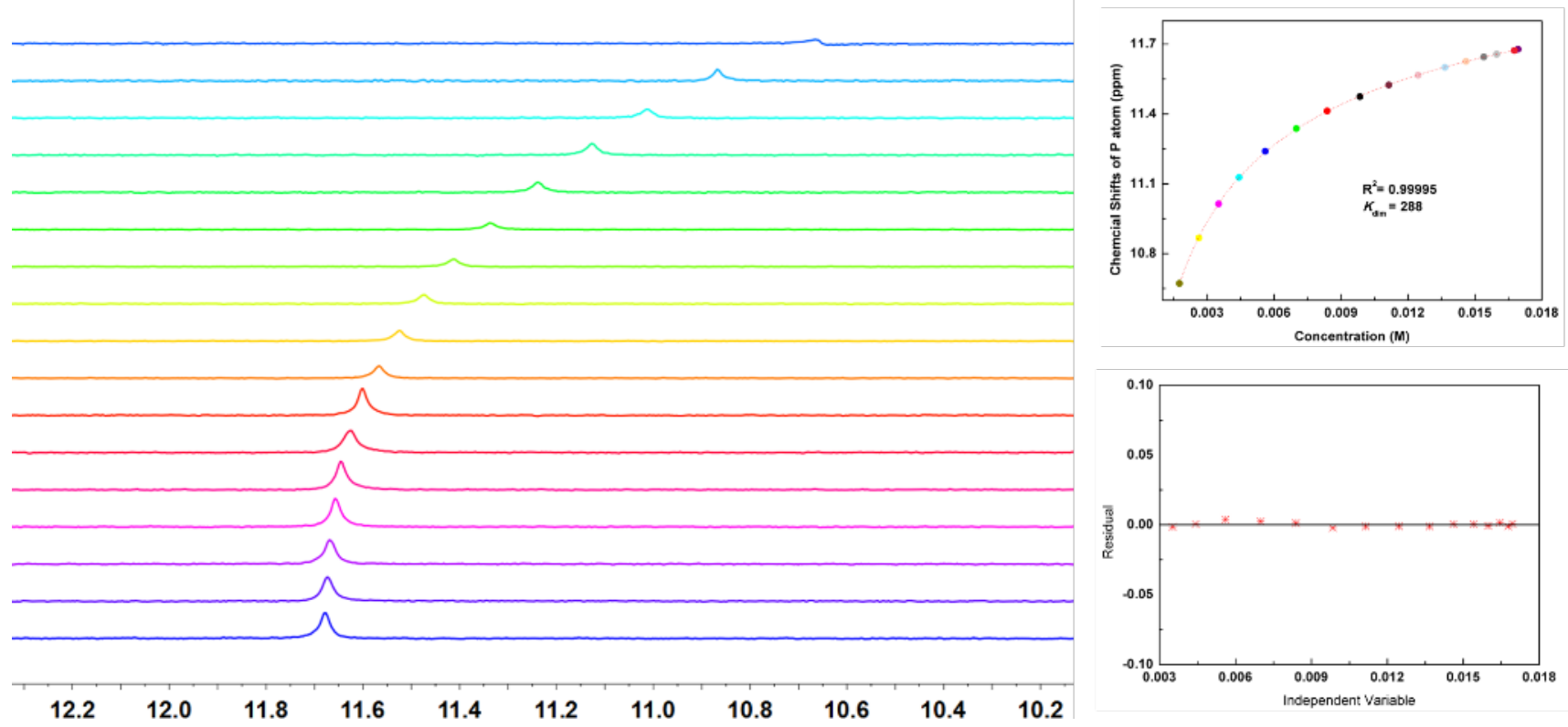

Figure S14. Stacked partial ${ }^{31} \mathrm{P}$ NMR spectra of compound $\mathbf{8 b}$ at different concentrations in water-saturated $\mathrm{CDCl}_{3}(298 \mathrm{~K})$. Determination of the dimerization constant of $\mathbf{8 b} \cdot \mathbf{8 b}$, fitting result based on chemical shifts of $\mathrm{P}$ atom. 

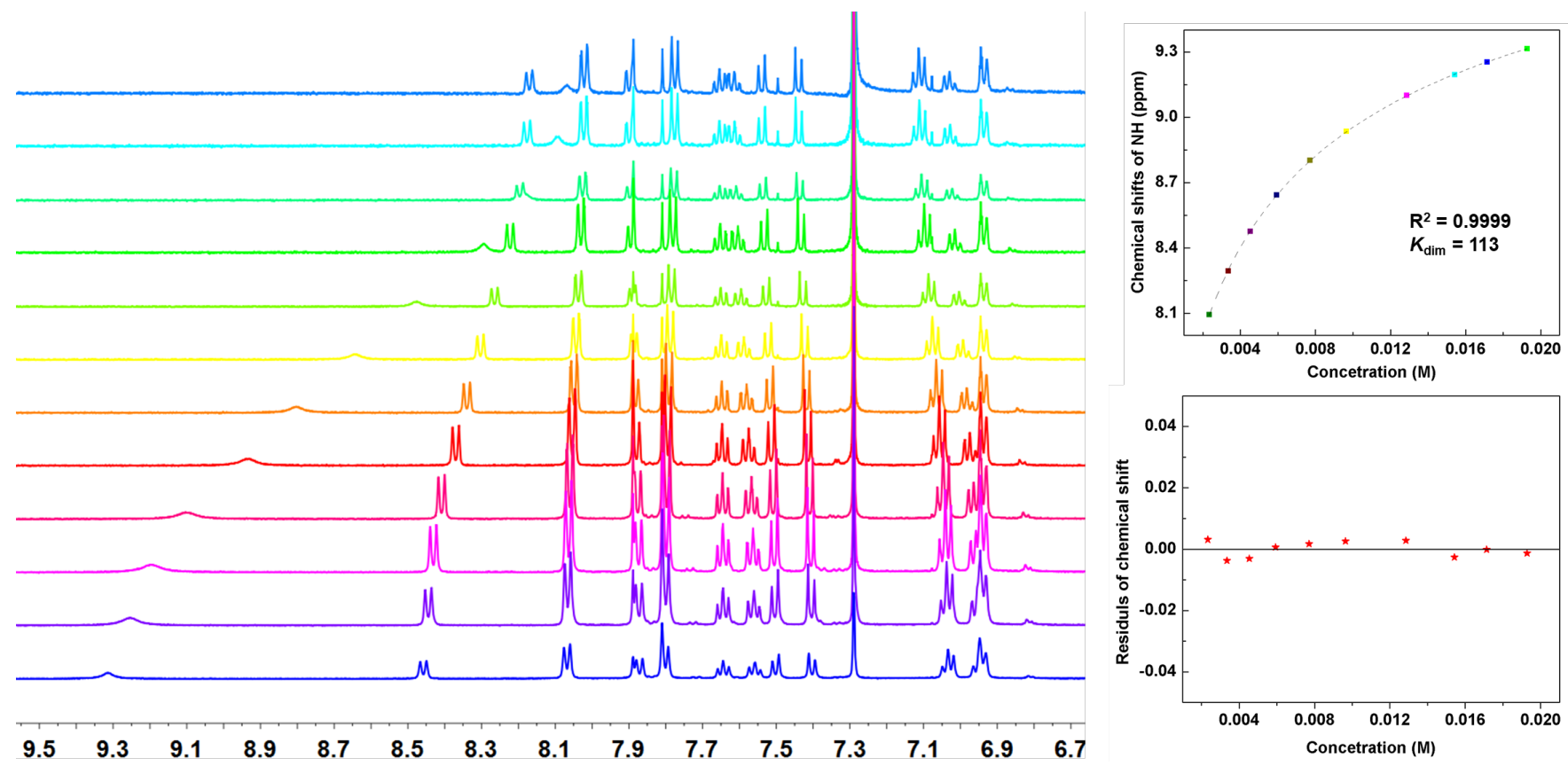

Figure S15. Stacked partial ${ }^{31} \mathrm{P}$ NMR spectra of compound $8 \mathbf{c}$ at different concentrations in water-saturated $\mathrm{CDCl}_{3}(298 \mathrm{~K})$. Determination of the dimerization constant of $\mathbf{8 c} \cdot \mathbf{8 c}$, fitting result based on chemical shifts of phosphonamidate $\mathrm{NH}$.

Self-dimerization of $\mathbf{8 d}$
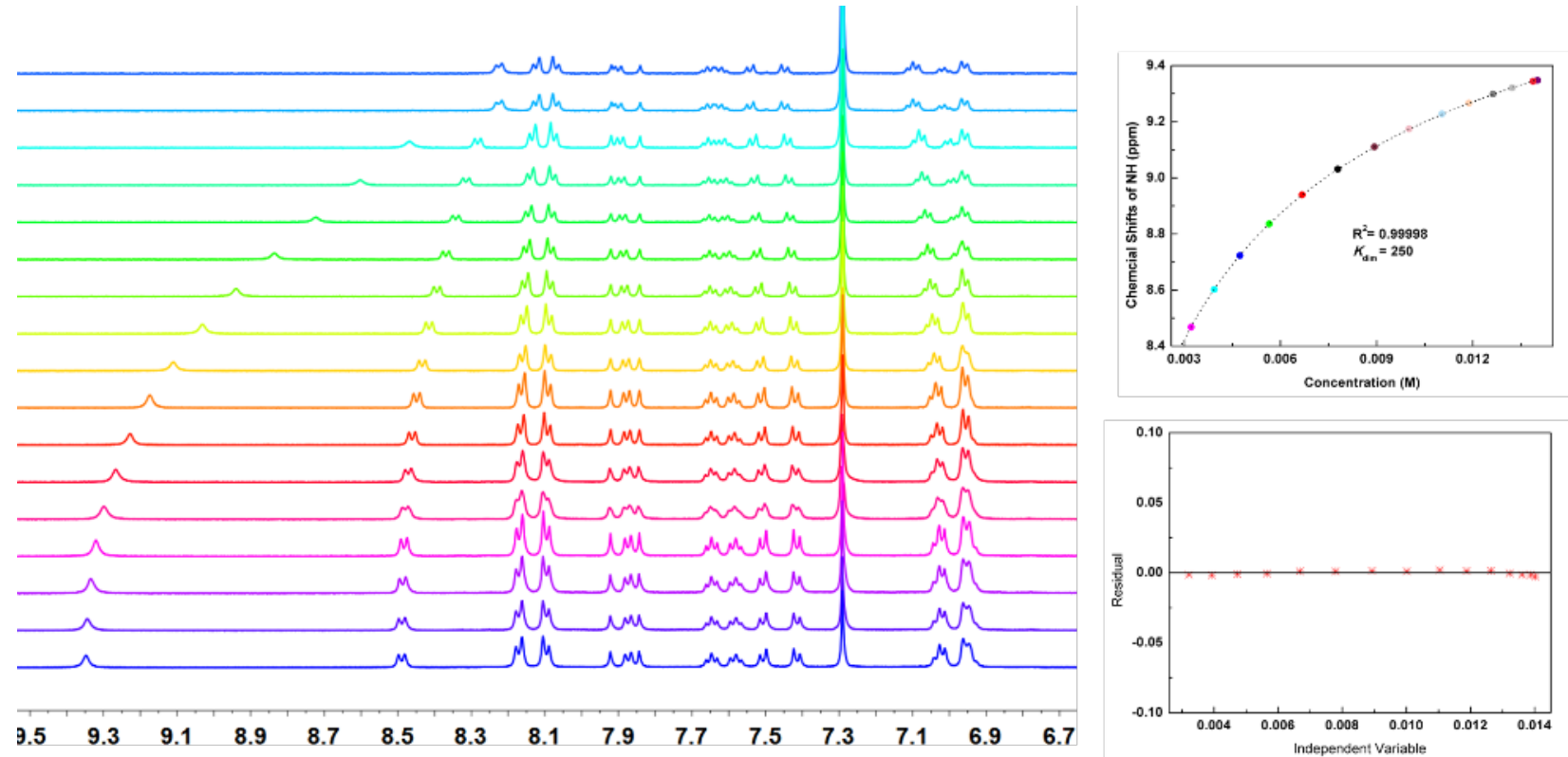

Figure S16. Stacked partial ${ }^{1} \mathrm{H}$ NMR spectra of compound 8d at different concentrations in water-saturated $\mathrm{CDCl}_{3}(298 \mathrm{~K})$. Determination of the dimerization constant of $\mathbf{8 d} \cdot \mathbf{8 d}$, fitting result based on chemical shifts of phosphonamidate $\mathrm{NH}$. 

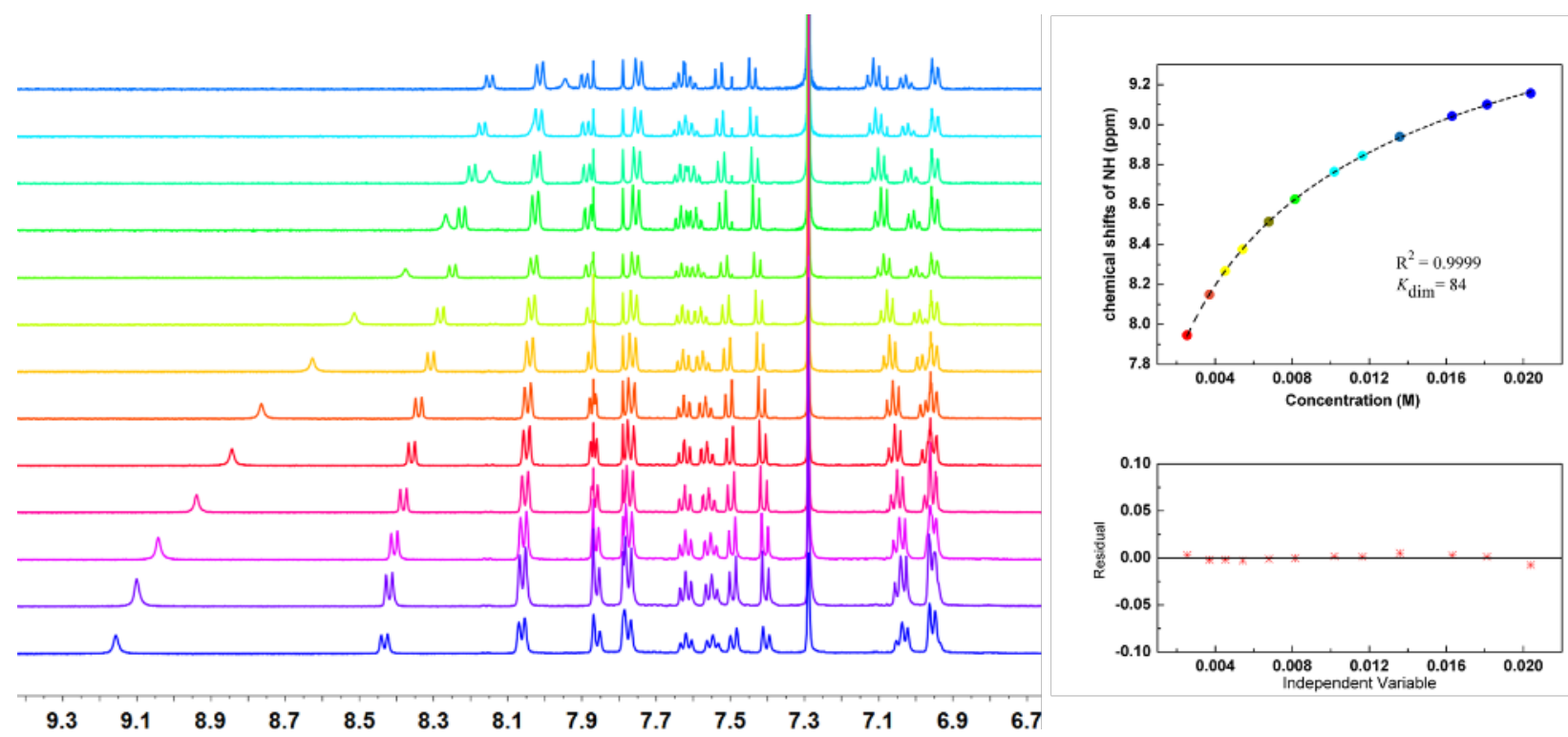

Figure S17. Stacked partial ${ }^{1} \mathrm{H}$ NMR spectra of compound 8e at different concentrations in water-saturated $\mathrm{CDCl}_{3}(298 \mathrm{~K})$. Determination of the dimerization constant of $\mathbf{8 e} \cdot \mathbf{8 e}$, fitting result based on chemical shifts of phosphonamidate $\mathrm{NH}$.

\section{Self-dimerization of $8 f$}
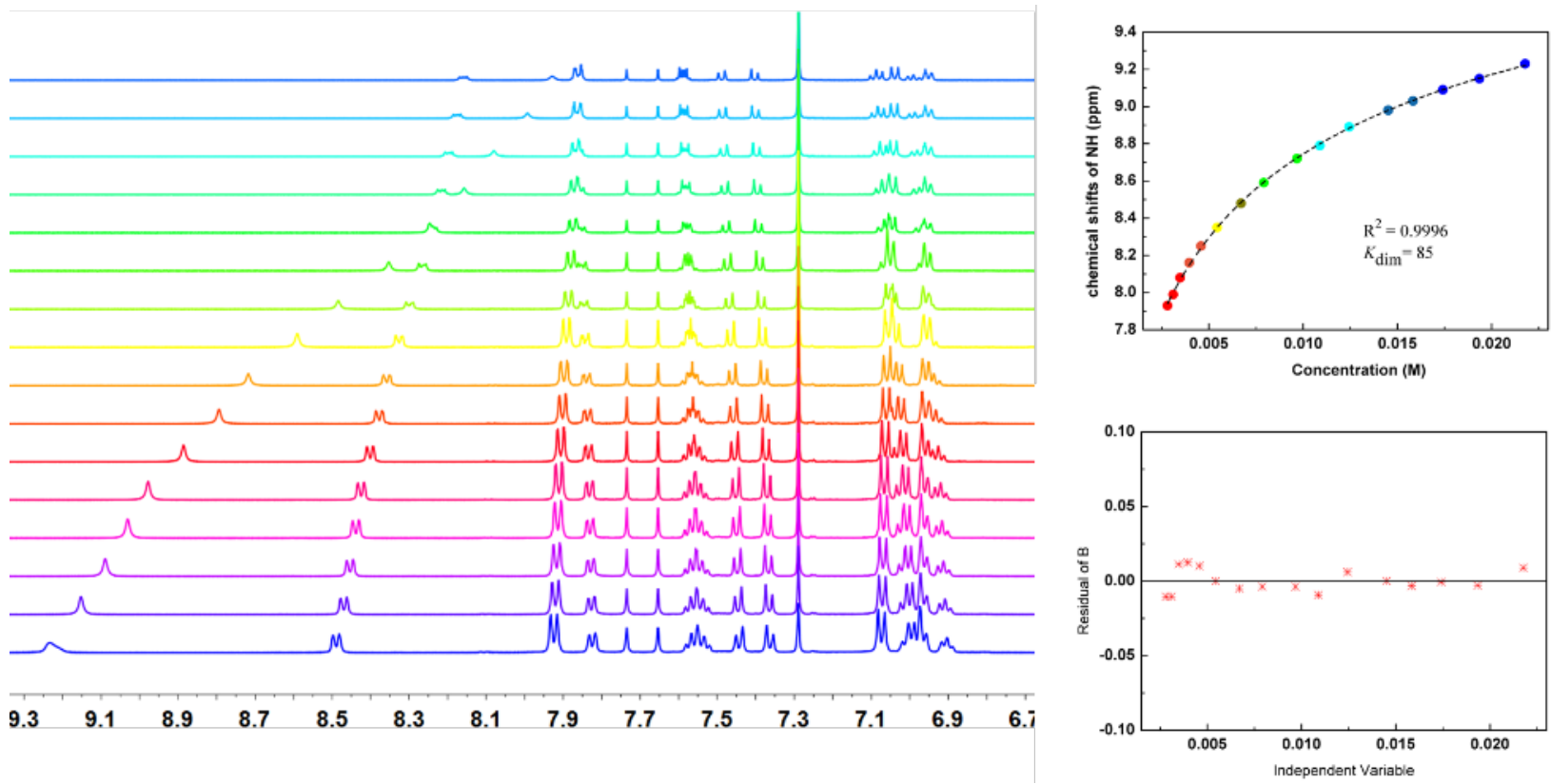

Figure S18. Stacked partial ${ }^{1} \mathrm{H}$ NMR spectra of compound $\mathbf{8 f}$ at different concentrations in water-saturated $\mathrm{CDCl}_{3}(298 \mathrm{~K})$. Determination of the dimerization constant of $\mathbf{8 f} \cdot \mathbf{8 f}$, fitting result based on chemical shifts of phosphonamidate $\mathrm{NH}$. 


\section{References}

1. Sheldrick, G. M.; Bruker/Siemens Area Detector Absorption Correction Program, Bruker AXS, Madison, WI, 1998.

2. Van der Sluis, P.; Spek, A. L. Acta Cryst. 1990, A46, 194-201.

3. Sheldrick, G. M. Acta Cryst. C 2015, 71, 3-8.

4. Brandenburg, K. DIAMOND Version 2.0, 1998.

5. Farrugia, L. J. Ortep 3 for windows. J. Appl. Cryst. 1997, 30, 565-566.

6. Wolff, S. K.; Grimwood, D. J.; McKinnon, J. J.; Jayatilaka, D.; Spackman, M. A. Crystal Explorer Version 1.5. University of Western Australia, Perth, Australia, 2007.

7. (a) Adamo, C.; Barone, V. J. Chem. Phys. 1999, 110, 6158-6169; (b) Bremond, E.; Savarese, M.; Su, N. Q.; Perez-Jimenez, A. J.; Xu, X.; Sancho-Garcia, J. C.; Adamo, C. J. Chem. Theory Comput. 2016, 12, 459-465.

8. Gaussian 09, Revision E.01, Frisch, M. J.; Trucks, G. W.; Schlegel, H. B.; Scuseria, G. E.; Robb, M. A.; Cheeseman, J. R.; Scalmani, G.; Barone, V.; Mennucci, B.; Petersson, G. A.; Nakatsuji, H.; Caricato, M.; Li, X.; Hratchian, H. P.; Izmaylov, A. F.; Bloino, J.; Zheng, G.; Sonnenberg, J. L.; Hada, M.; Ehara, M.; Toyota, K.; Fukuda, R.; Hasegawa, J.; Ishida, M.; Nakajima, T.; Honda, Y.; Kitao, O.; Nakai, H.; Vreven, T.; Montgomery, J. A., Jr.; Peralta, J. E.; Ogliaro, F.; Bearpark, M.; Heyd, J. J.; Brothers, E.; Kudin, K. N.; Staroverov, V. N.; Kobayashi, R.; Normand, J.; Raghavachari, K.; Rendell, A.; Burant, J. C.; Iyengar, S. S.; Tomasi, J.; Cossi, M.; Rega, N.; Millam, J. M.; Klene, M.; Knox, J. E.; Cross, J. B.; Bakken, V.; Adamo, C.; Jaramillo, J.; Gomperts, R.; Stratmann, R. E.; Yazyev, O.; Austin, A. J.; Cammi, R.; Pomelli, C.; Ochterski, J. W.; Martin, R. L.; Morokuma, K.; Zakrzewski, V. G.; Voth, G. A.; Salvador, P.; Dannenberg, J. J.; Dapprich, S.; Daniels, A. D.; Farkas, Ö.; Foresman, J. B.; Ortiz, J. V.; Cioslowski, J.; Fox, D. J. Gaussian, Inc., Wallingford CT, 2013.

9. (a) Miertus, S.; Scrocco, E.; Tomasi, J. Chem. Phys. 1981, 55, 117-129; (b) Barone, V.; Cossi, M.; Tomasi, J. J. Comput. Chem. 1998, 19, 404-417.

10. Zhao, Y.; Truhlar, D. G. Theor. Chem. Acc. 2008, 120, 215-241.

11. Schuafer, A.; Huber, C.; Ahlrichs, R. J. Chem. Phys. 1994, 100, 5829-5835. 
12. (a) Zheng, J.; Xu, X.; Truhlar, D. G. Theor. Chem. Acc. 2010, 128, 295-305; (b) Weigend, F.; Ahlrichs, R. Phys. Chem. Chem. Phys. 2005, 7, 3297-3305.

13. Boys, S. F.; Bernardi, F. Mol. Phys. 1970, 19, 553-566.

14. Bader, R. F. W. Atoms in Molecules, A Quantum Theory; Oxford University Press: Oxford, 1990.

15. Espinosa, E.; Molins, E.; Lecomte, C. Chem. Phys. Lett. 1998, 285, 170-173.

16. (a) Lu, T.; Chen, F. J. Comput. Chem. 2012, 33, 580-592; (b) Lu, T.; Chen, F. J. Mol. Graphics Modell. 2012, 38, 314-323.

17. (a) Phipps, M. J. S.; Fox, T.; Tautermannb, C. S.; Skylaris, C.-K. Chem. Soc. Rev. 2015, 44, 3177-3211; (b) Jeziorski, B.; Moszynski, R.; Szalewicz, K. Chem. Rev. 1994, 94, 1887-1930;

(c) Hohenstein, E. G.; Sherrill, C. D. WIREs Comput. Mol. Sci. 2012, 2, 304-326.

18. Parker, T. M.; Burns, L. A.; Parrish, R. M.; Ryno, A. G.; Sherrill, C. D. J. Chem. Phys. 2014, 140, 094106-094116.

19. Parrish, R. M.; Burns, L. A.; Smith, D. G. A.; Simmonett, A. C.; DePrince, A. E., III; Hohenstein, E. G.; Bozkaya, U.; Sokolov, A. Y.; Remigio, R. D.; Richard, R. M.; Gonthier, J. F.; James, A. M.; McAlexander, H. R.; Kumar, A.; Saitow, M.; Wang, X.; Pritchard, B. P.; Verma, P.; Schaefer III, H. F.; Patkowski, K.; King, R. A.; Valeev, E. F.; Evangelista, F. A.; Turney, J. M.; Crawford, T. D.; Sherrill, C. D. J. Chem. Theory Comput. 2017, 13, 3185-3197.

20. Thordarson, P. Chem. Soc. Rev. 2011, 40, 1305-1323. 


\section{Copies of NMR Spectra for New Compounds}
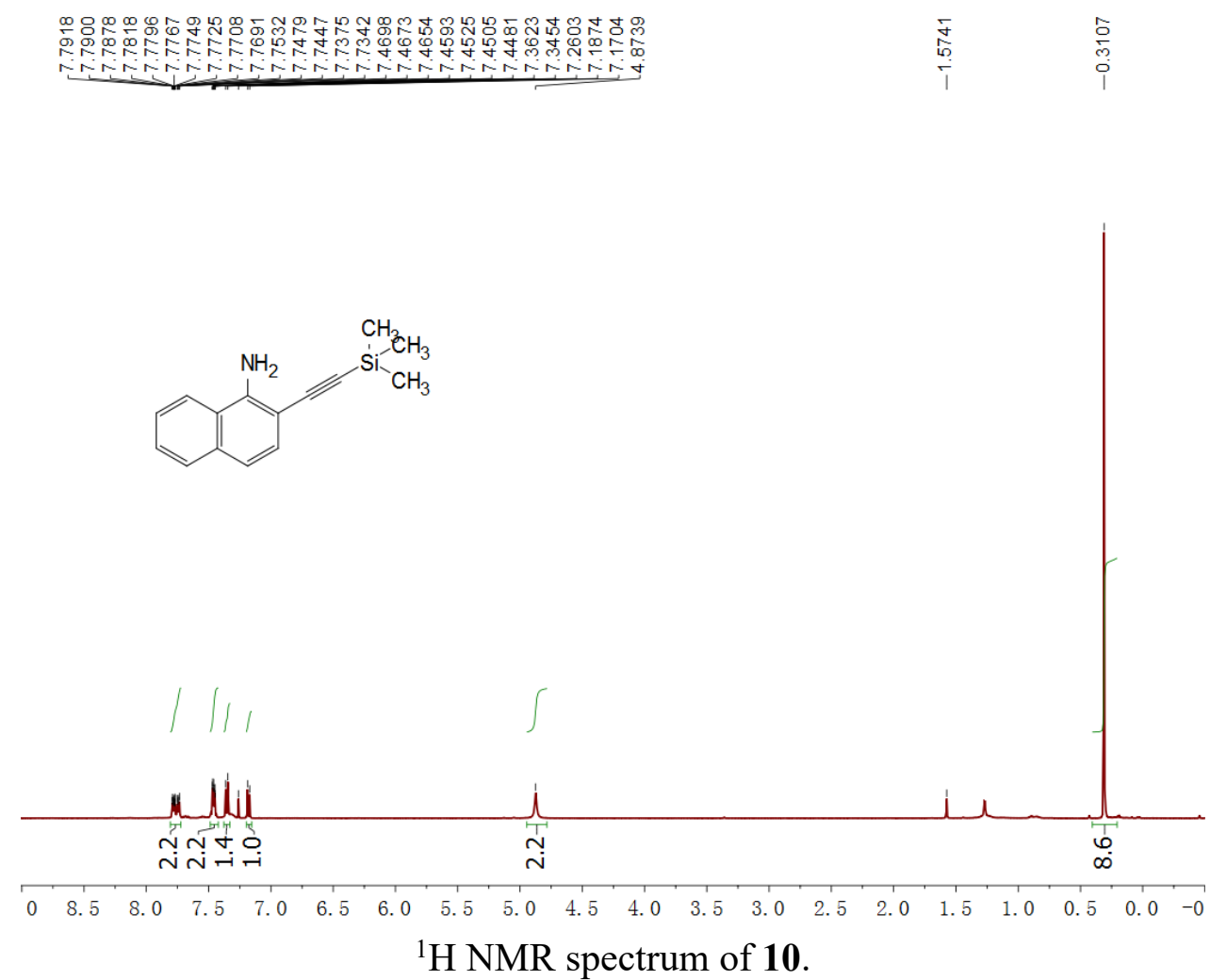

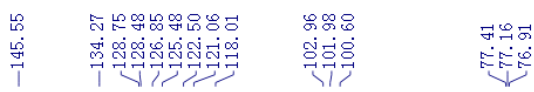

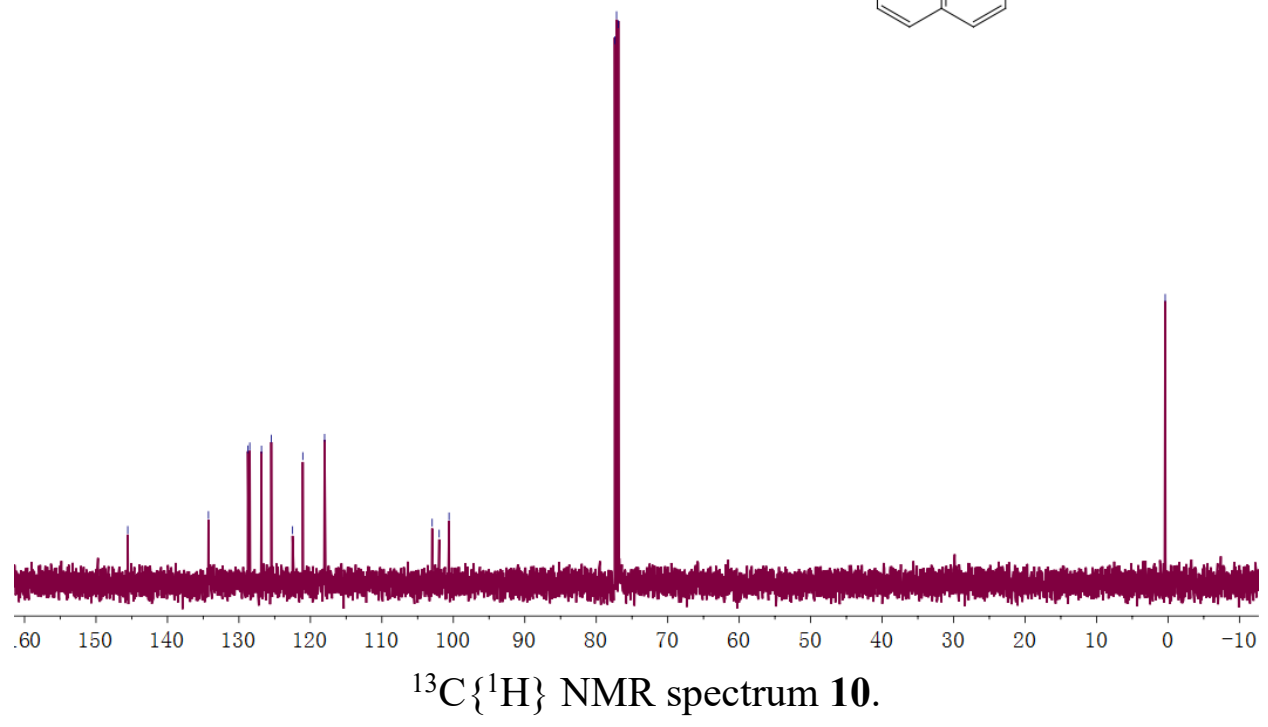



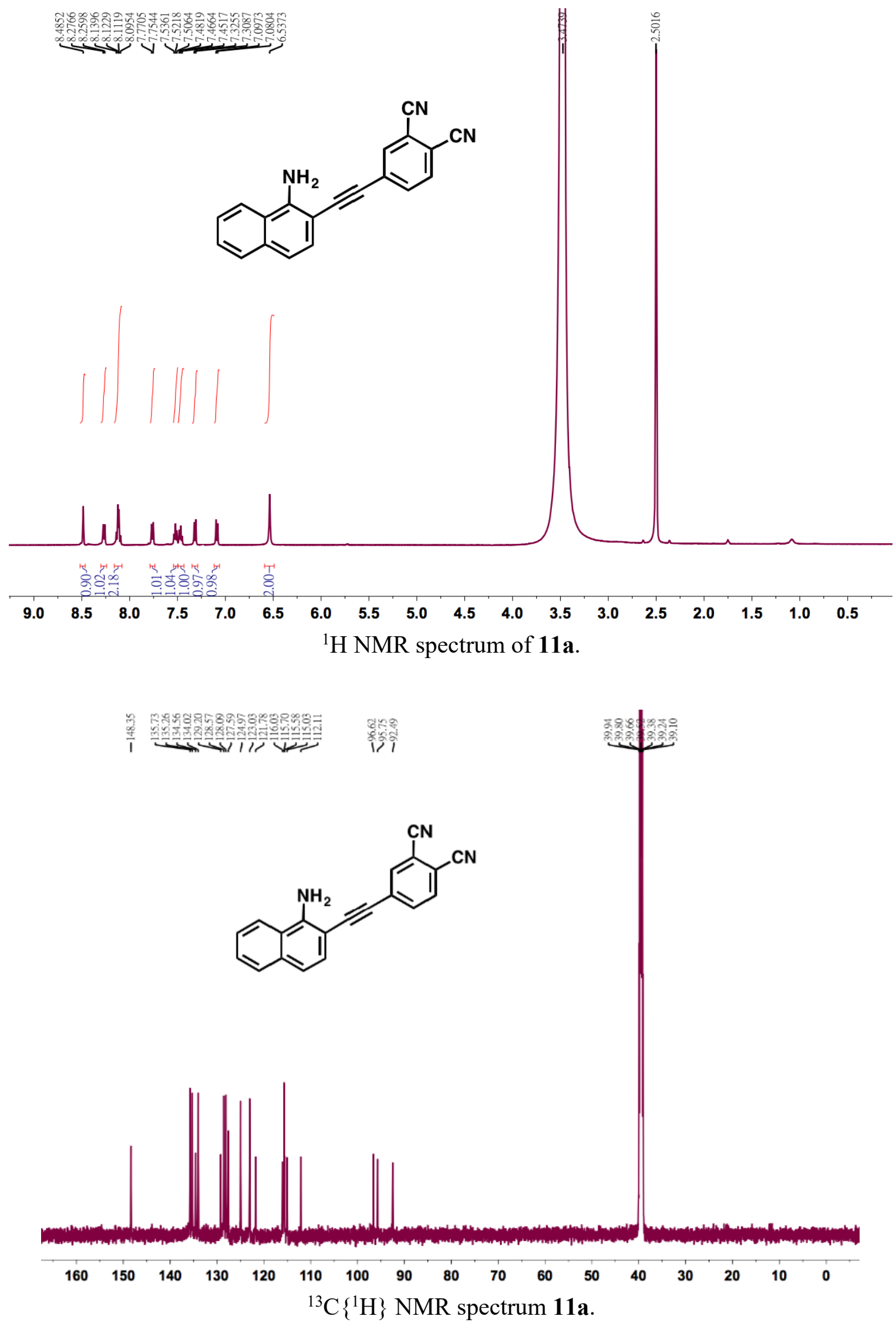

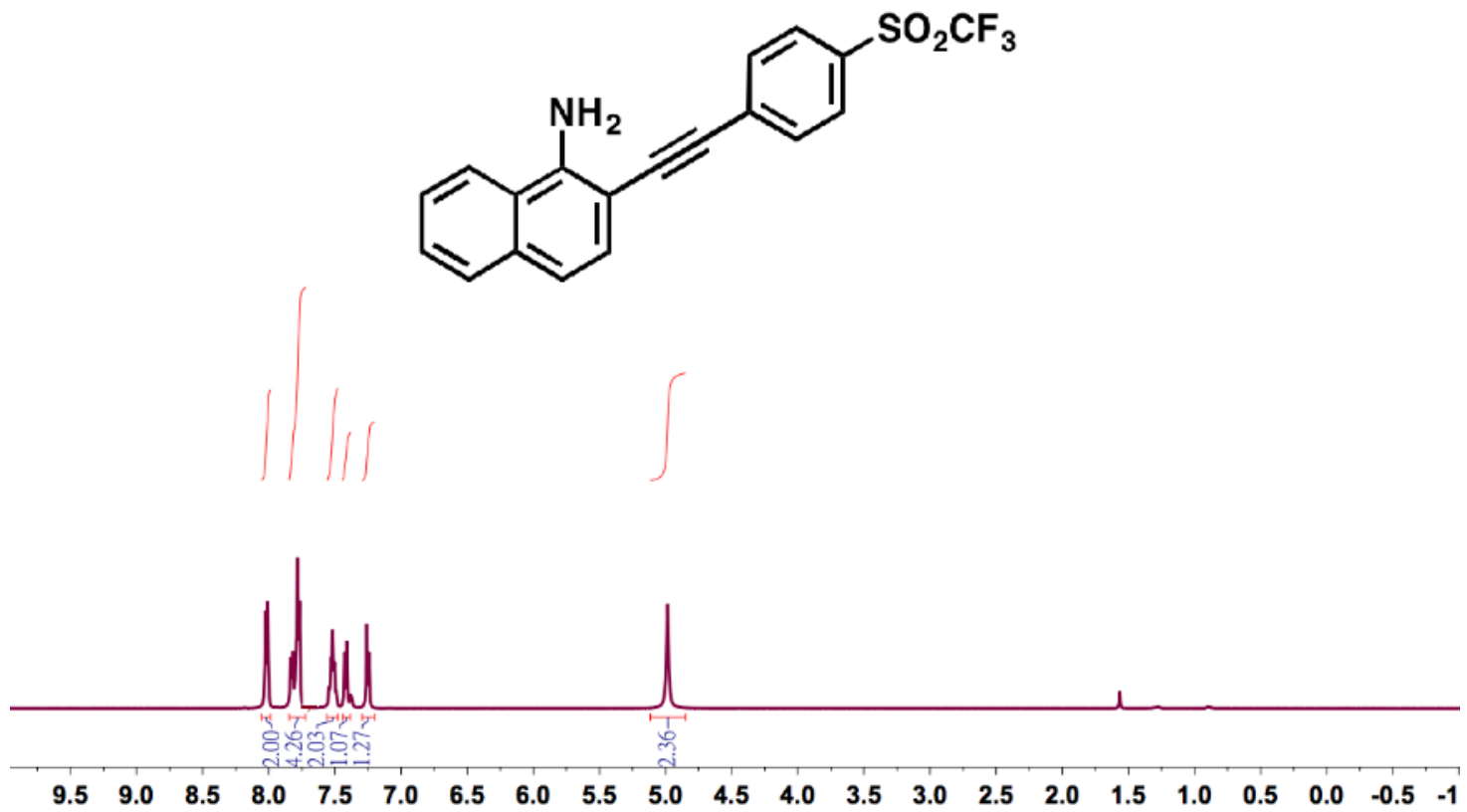

${ }^{1} \mathrm{H}$ NMR spectrum of $\mathbf{1 1 b}$.

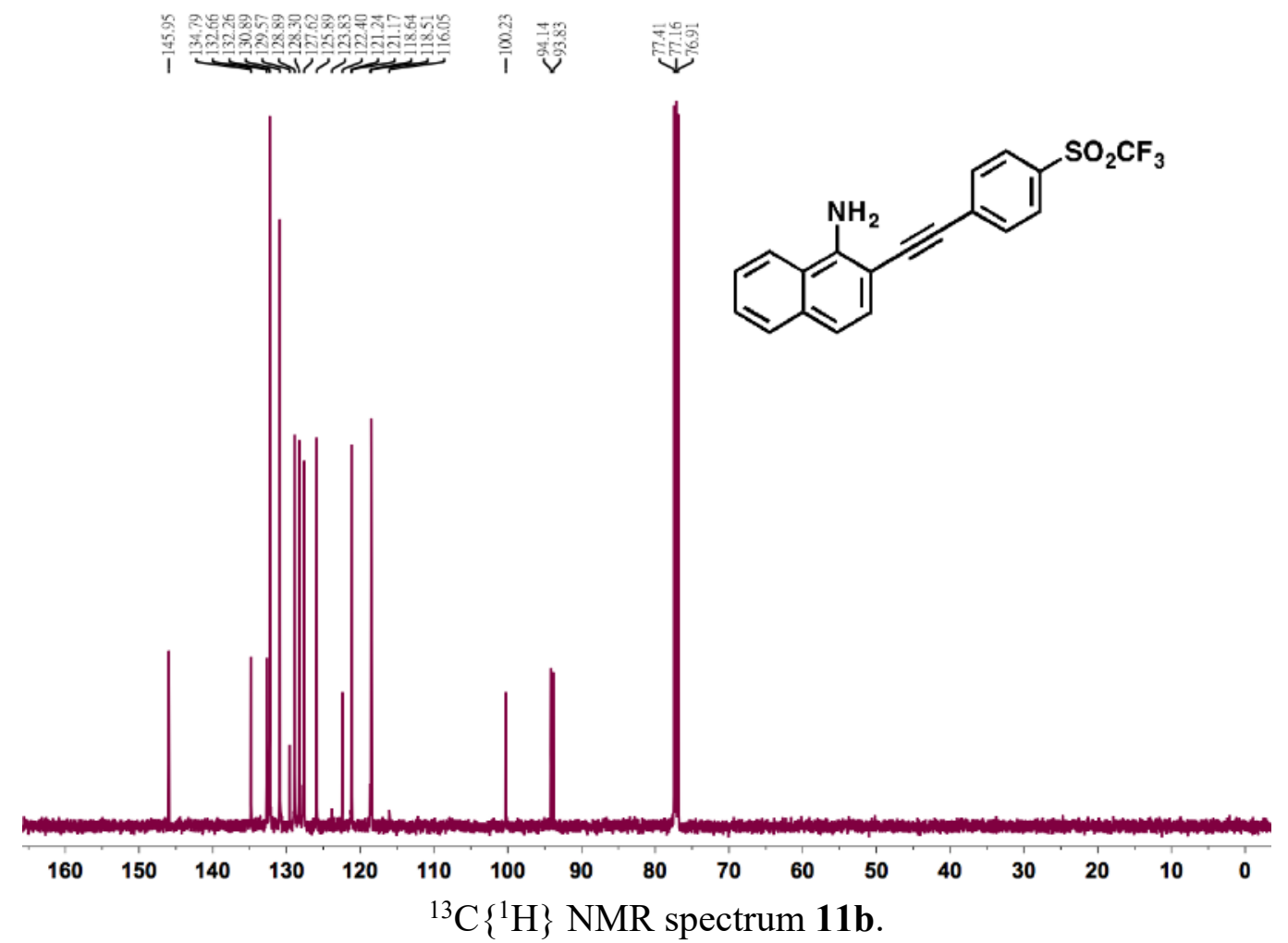




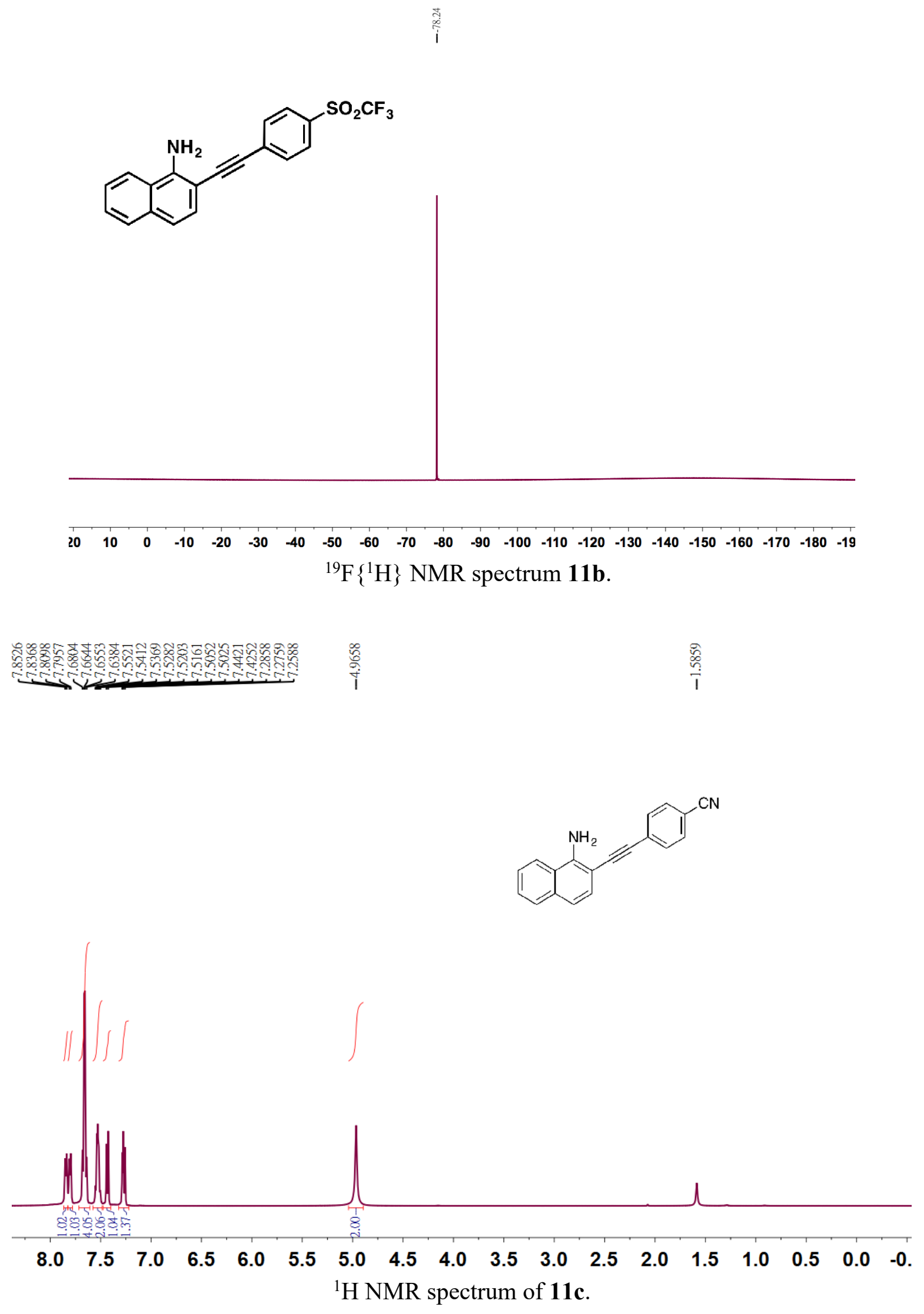



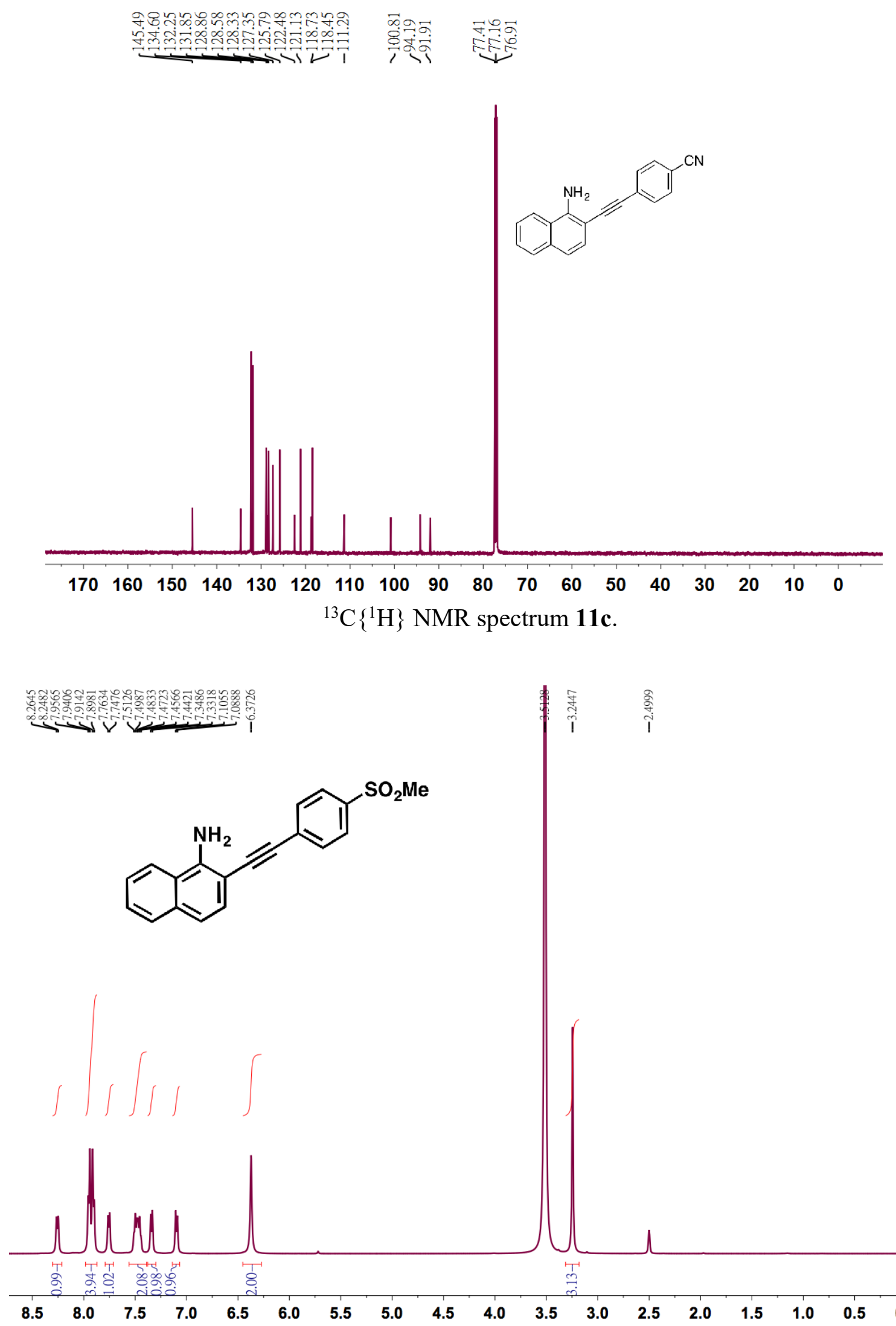

${ }^{1} \mathrm{H}$ NMR spectrum of $11 \mathrm{~d}$. 


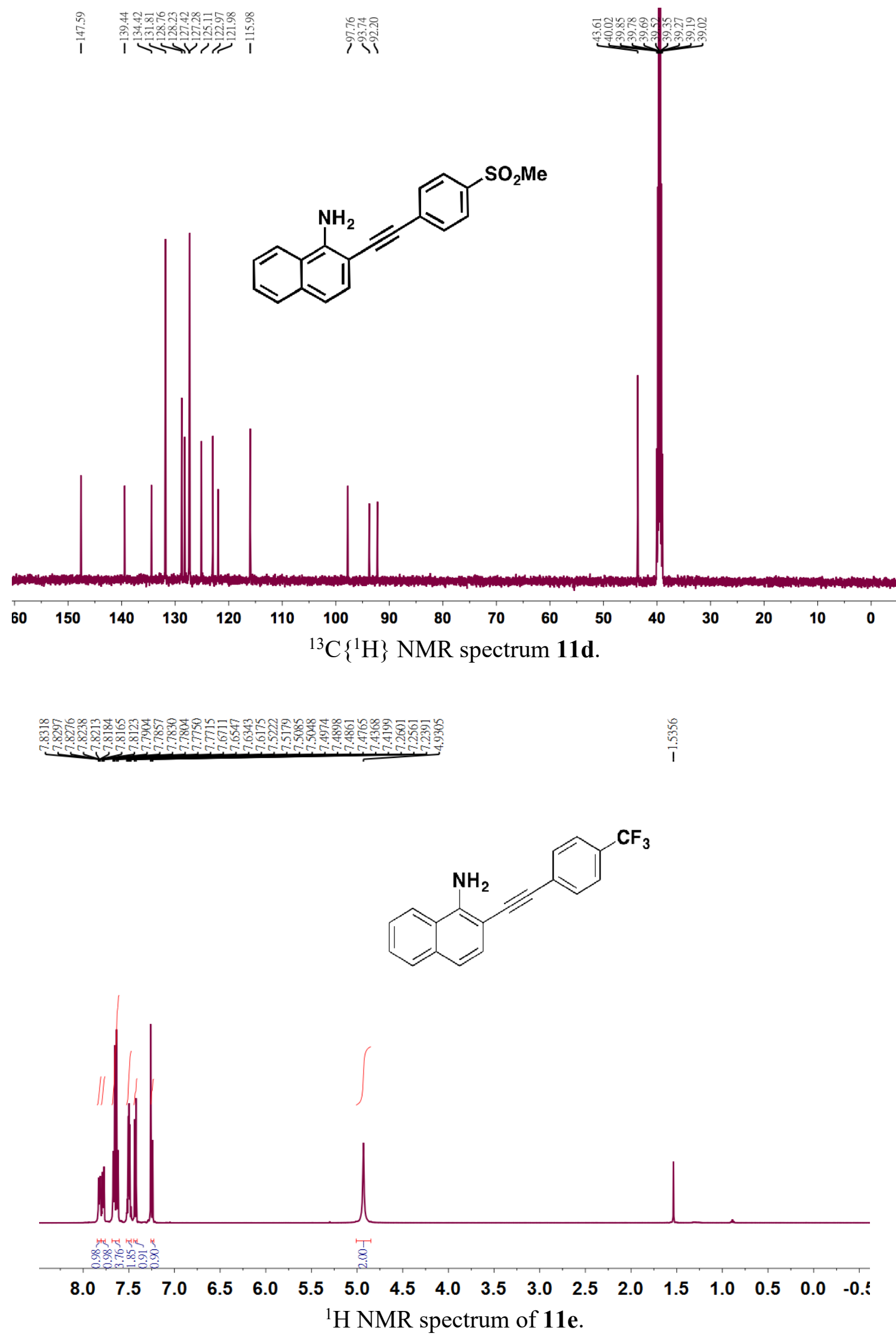



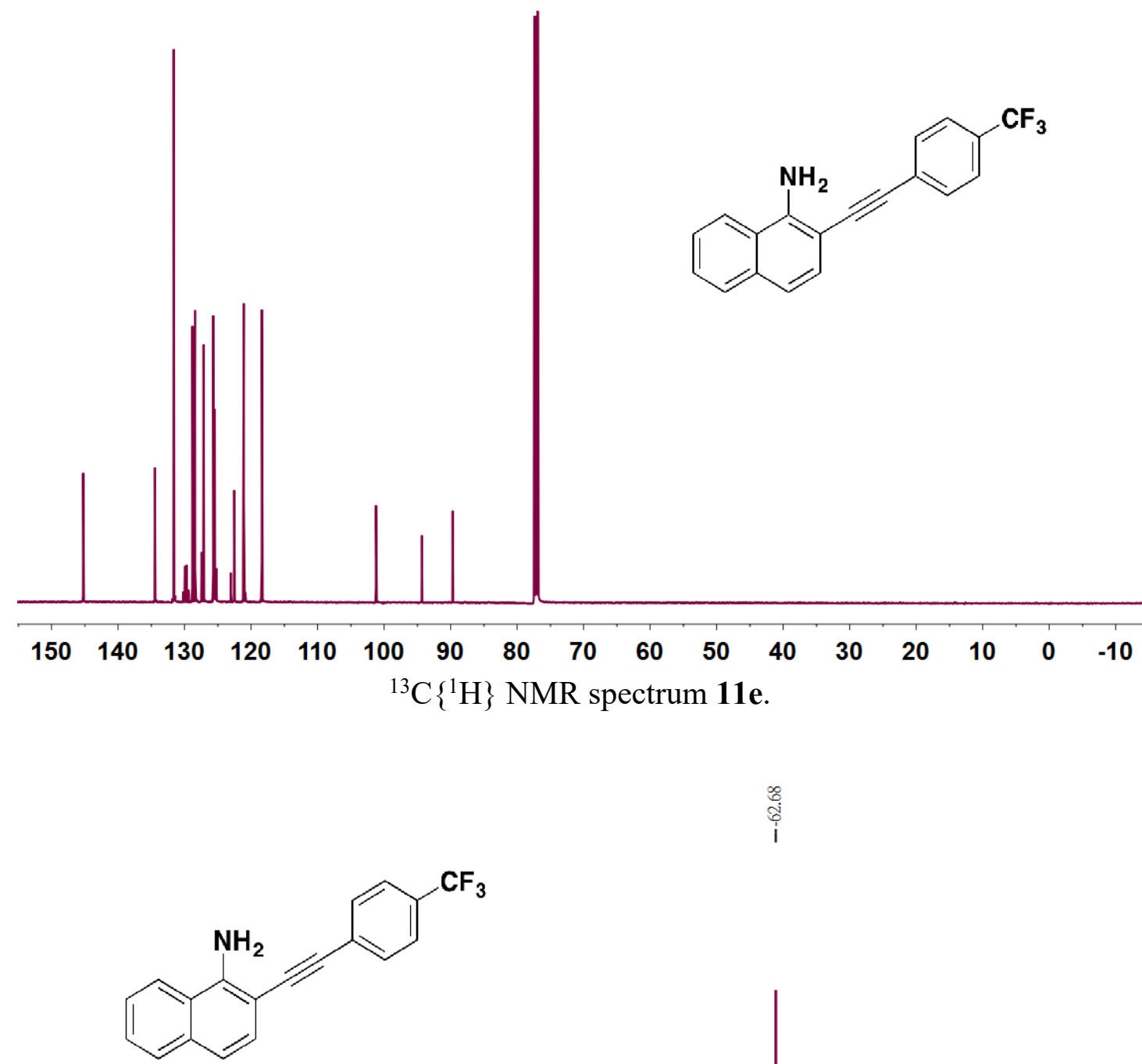

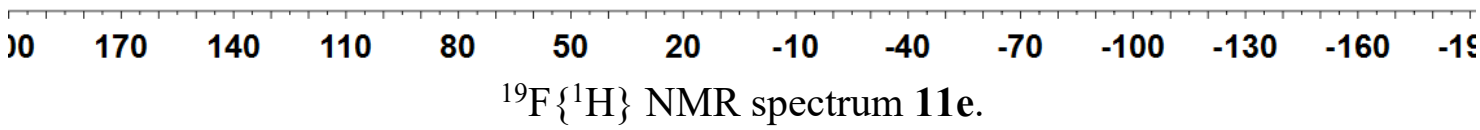



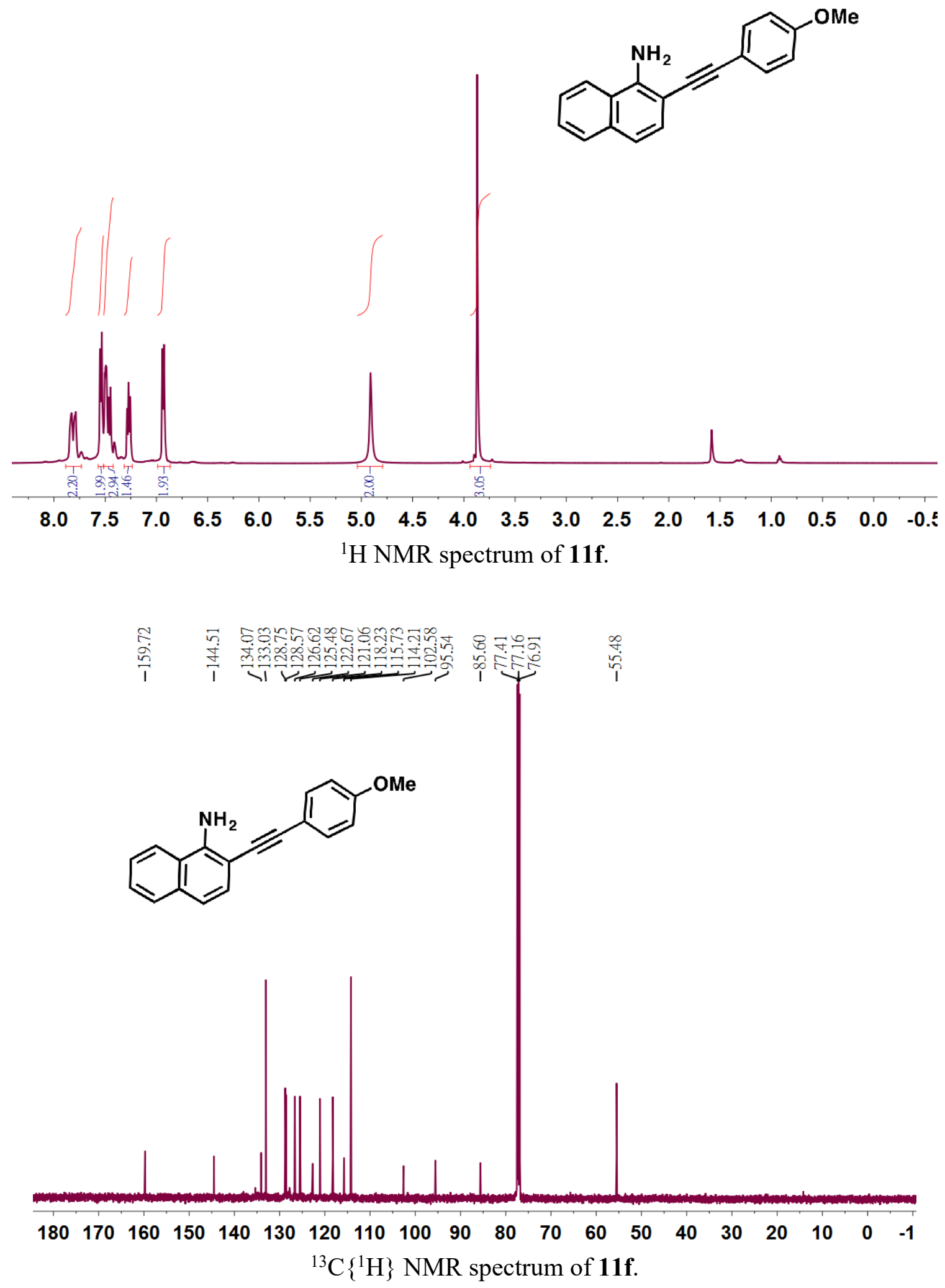

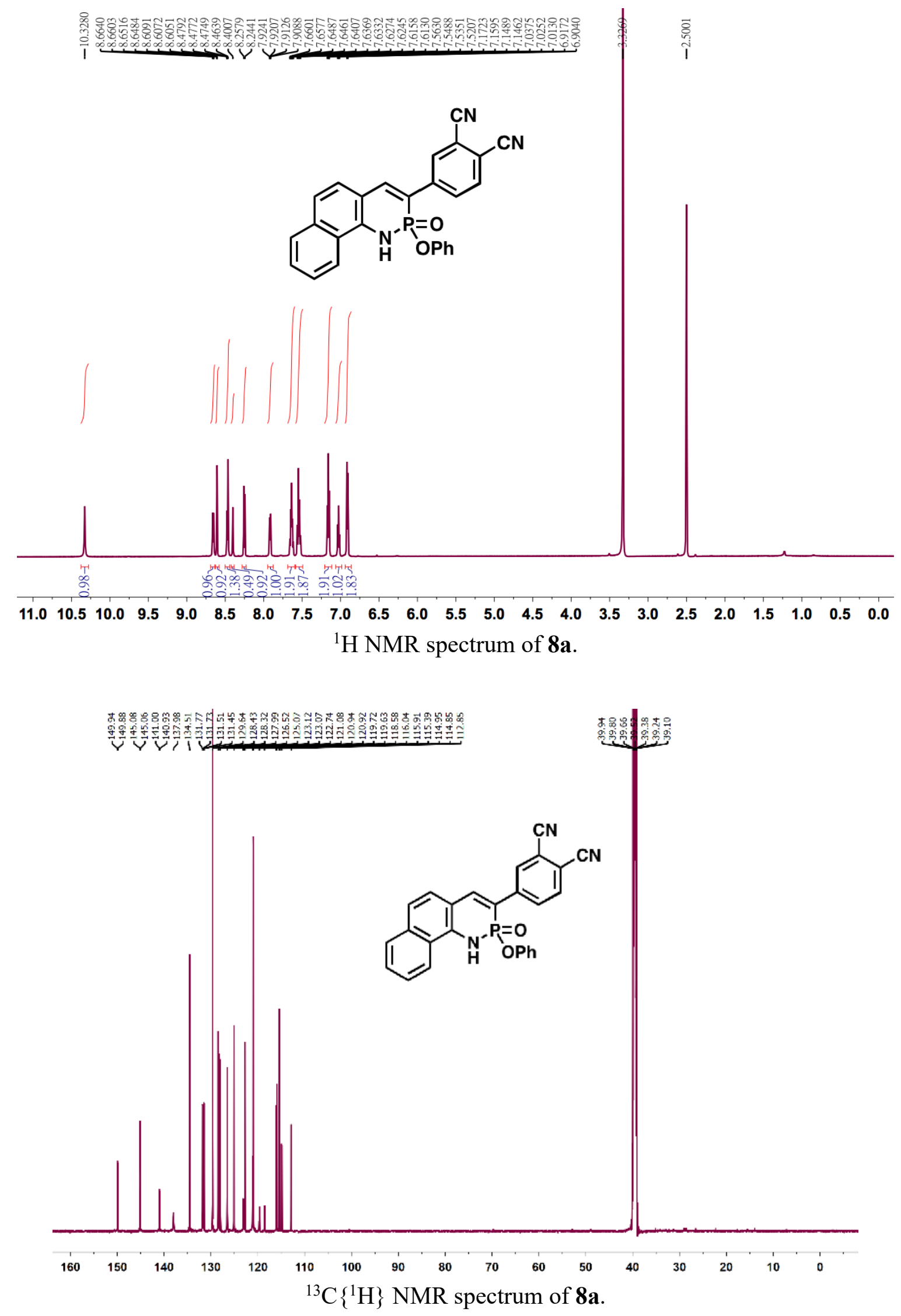


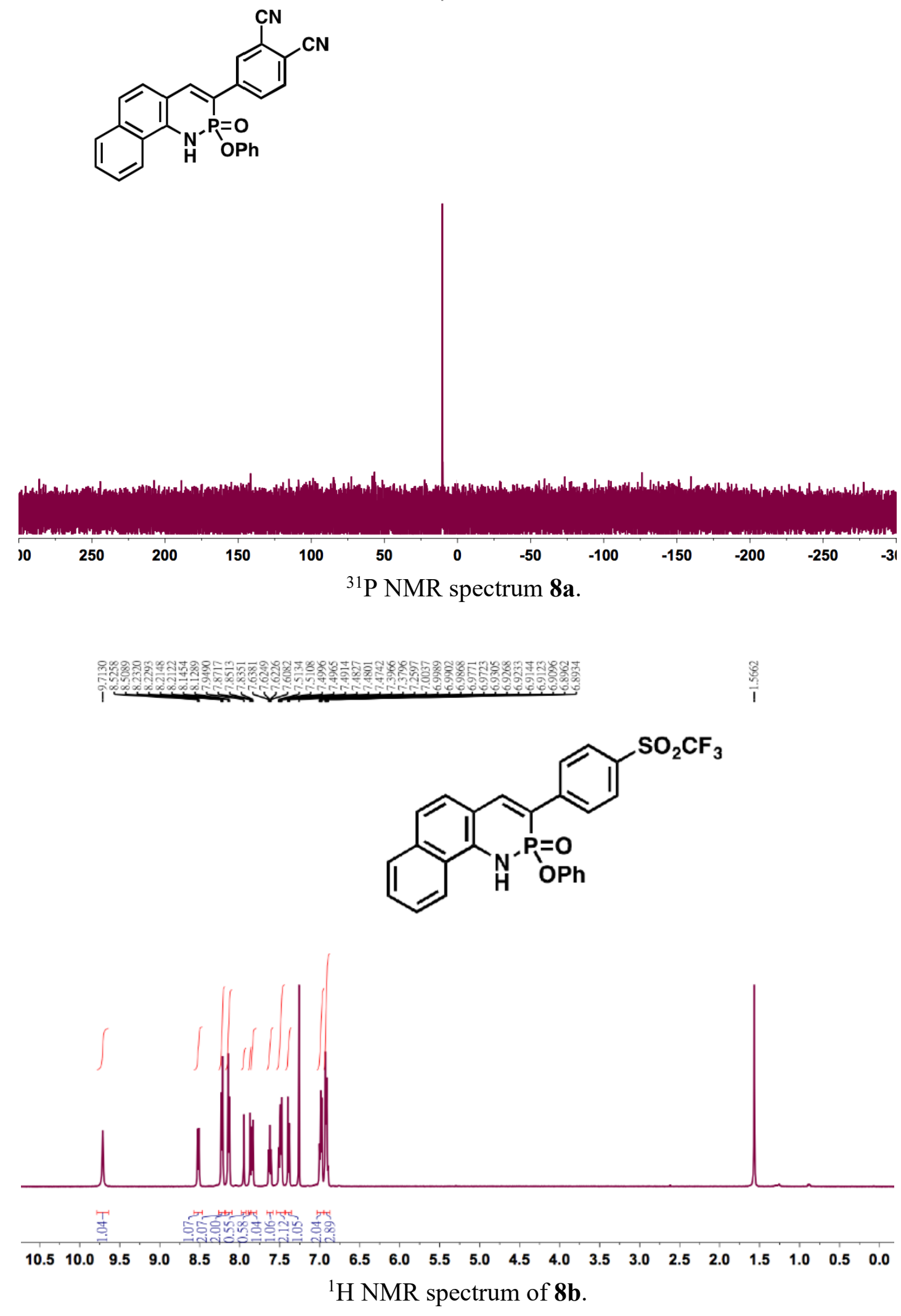




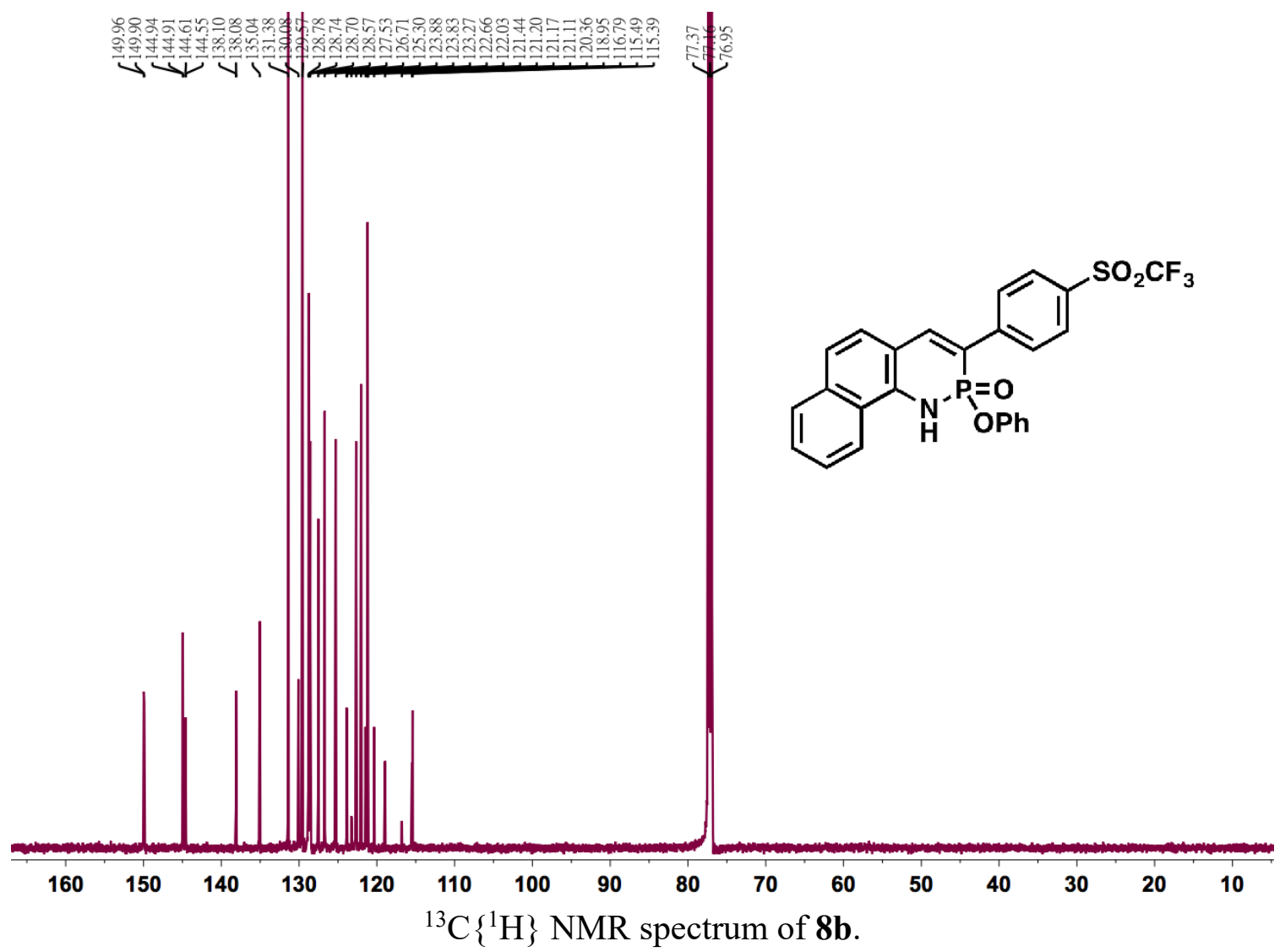

원<smiles>O=P1(Oc2ccccc2)Nc2c(ccc3ccccc23)C=C1c1ccc(S(=O)(=O)OC(F)(F)F)cc1</smiles>

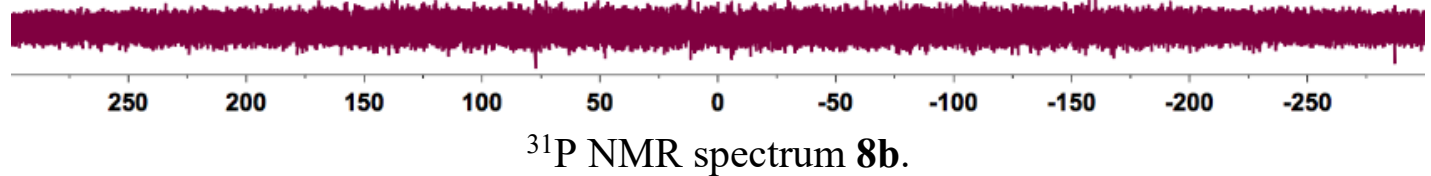




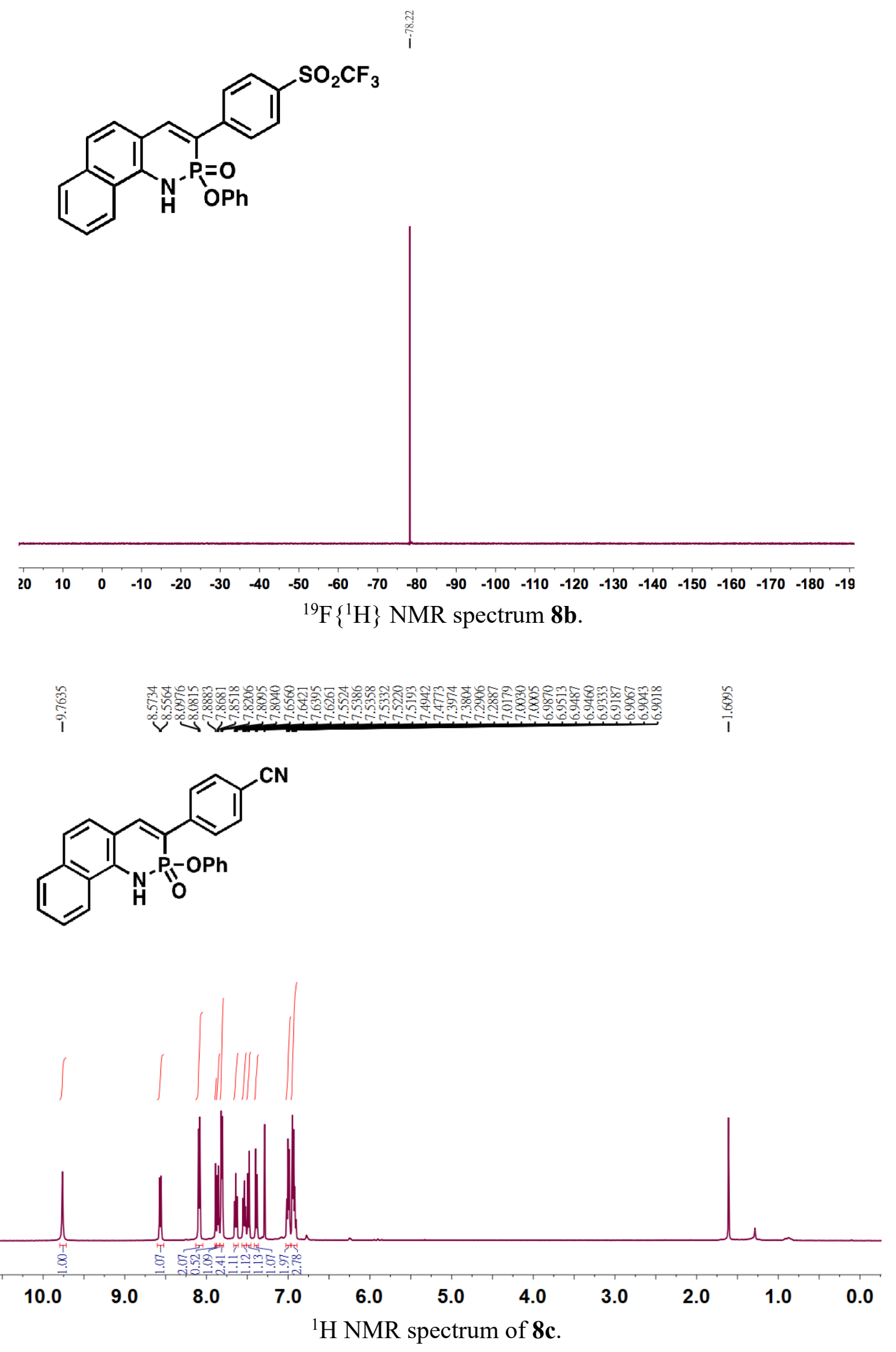




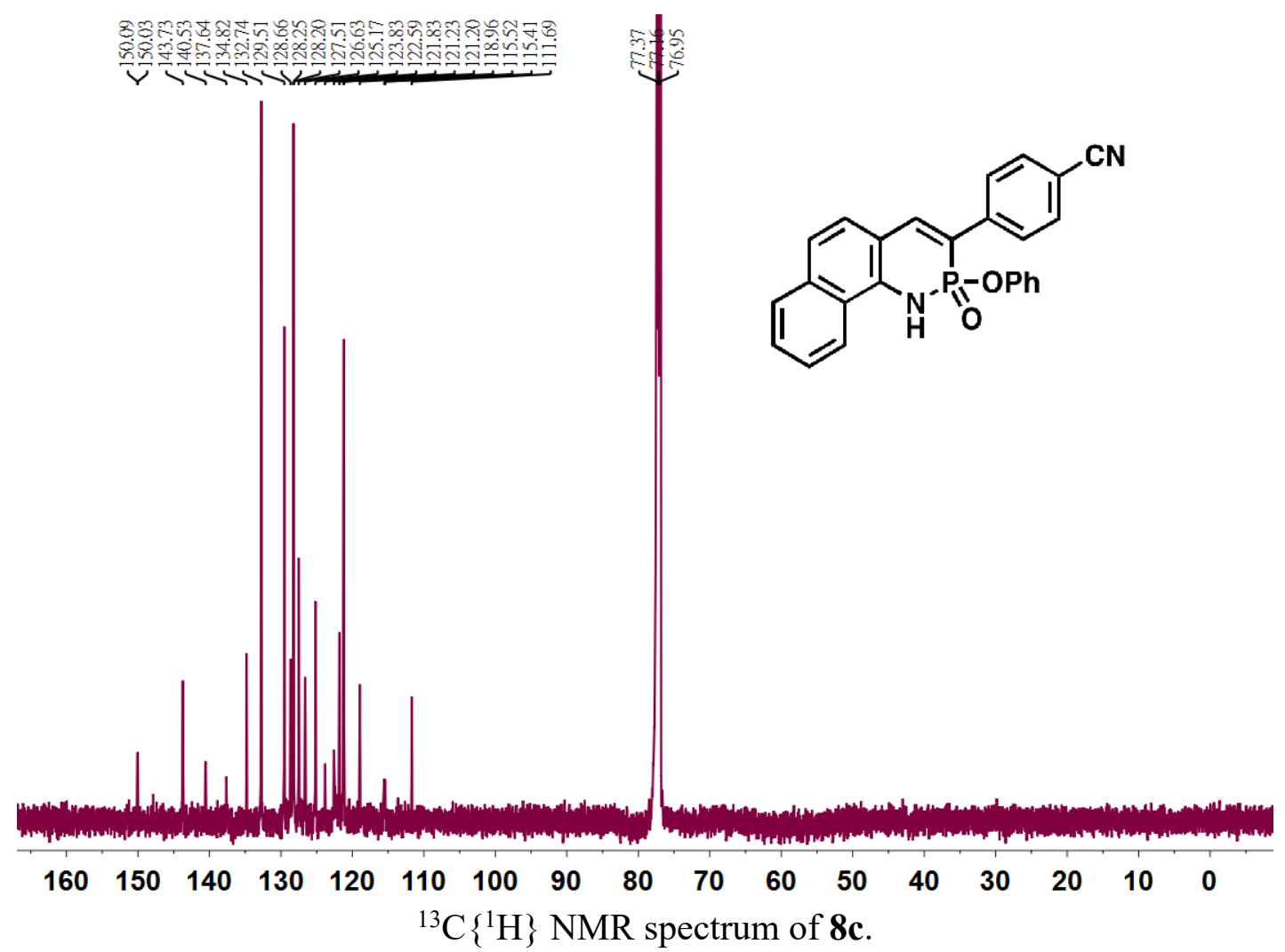

긿<smiles>N#Cc1ccc(C2=Cc3ccc4ccccc4c3NP2(=O)O)cc1</smiles>

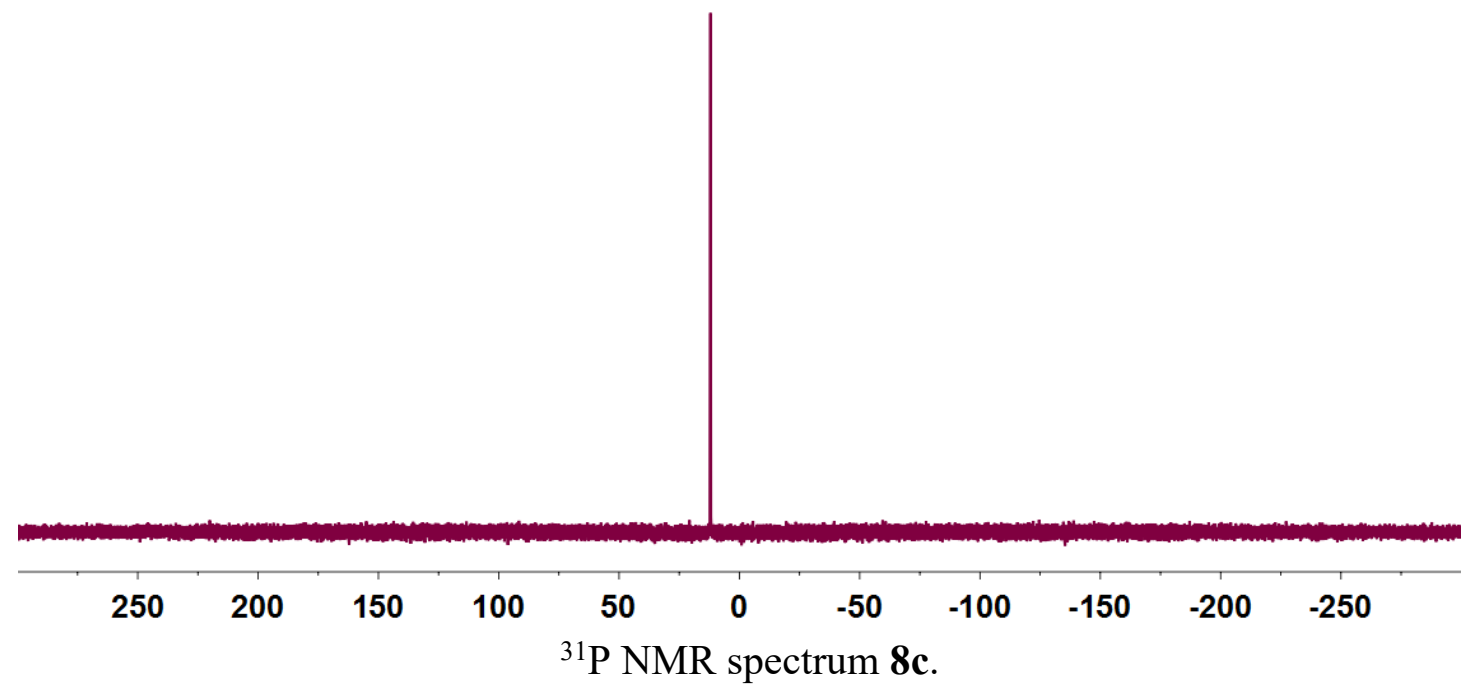



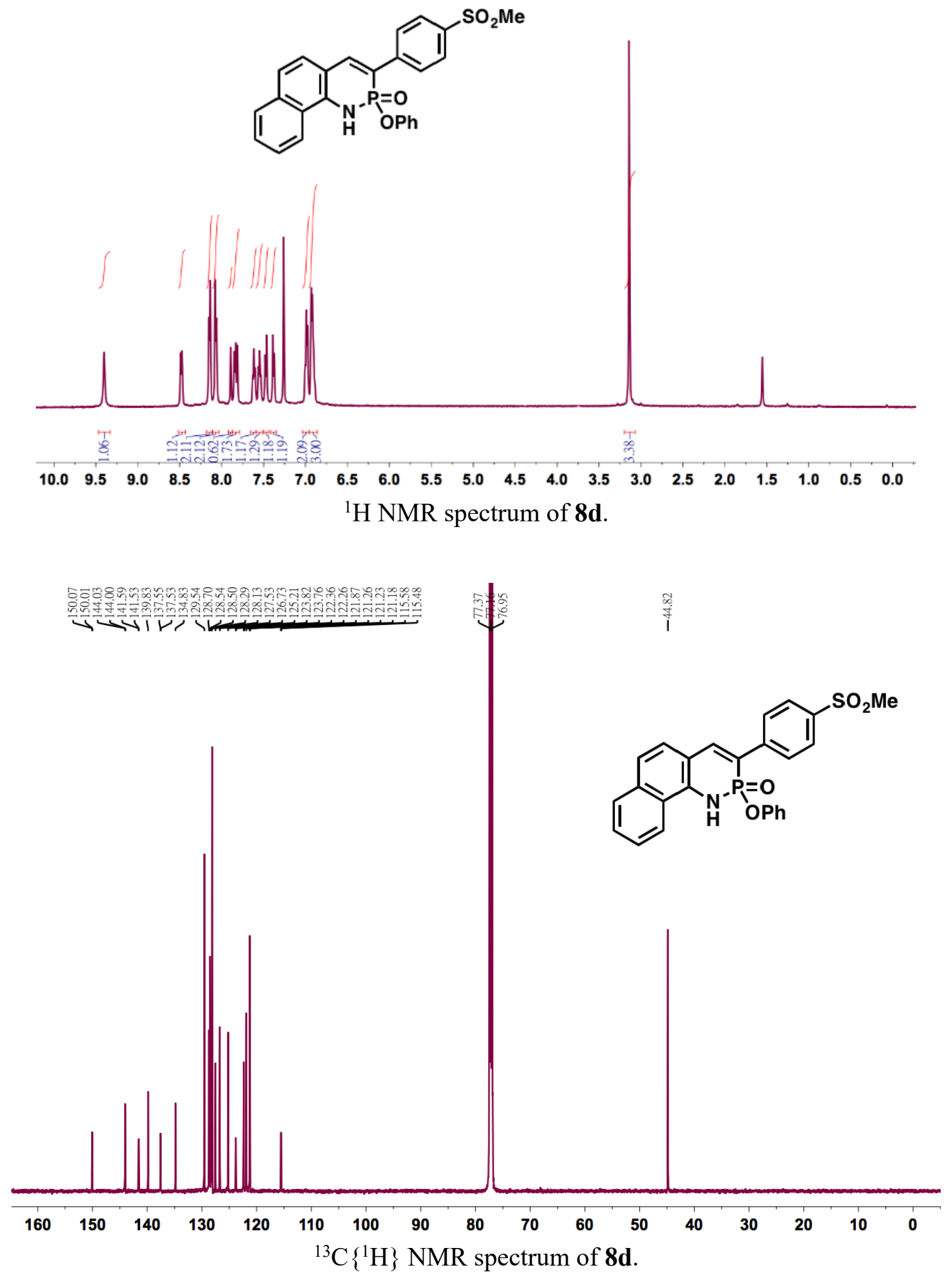


\section{留}<smiles>COS(=O)(=O)c1ccc(C2=Cc3ccc4ccccc4c3NP2(=O)c2ccccc2)cc1</smiles>
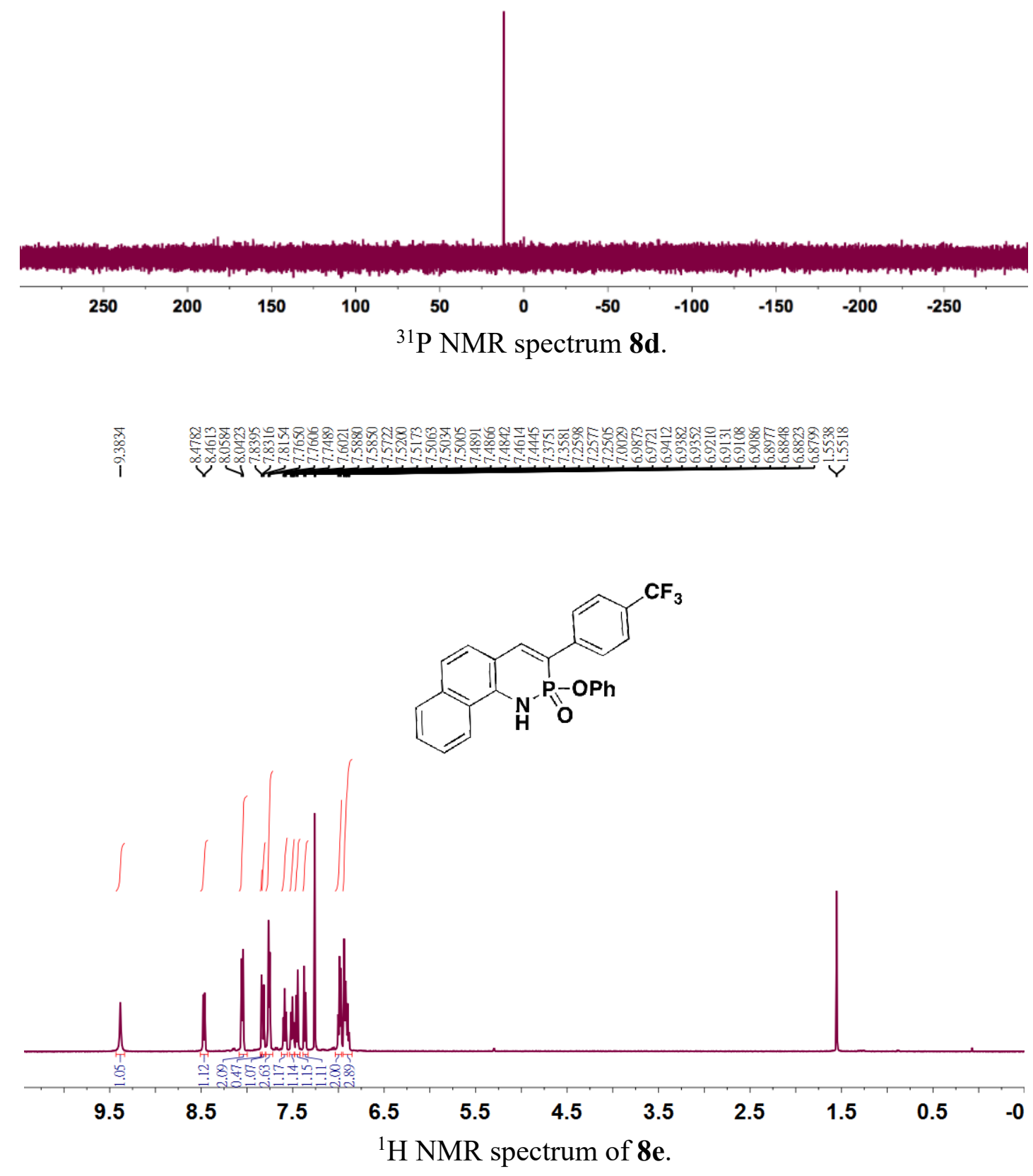


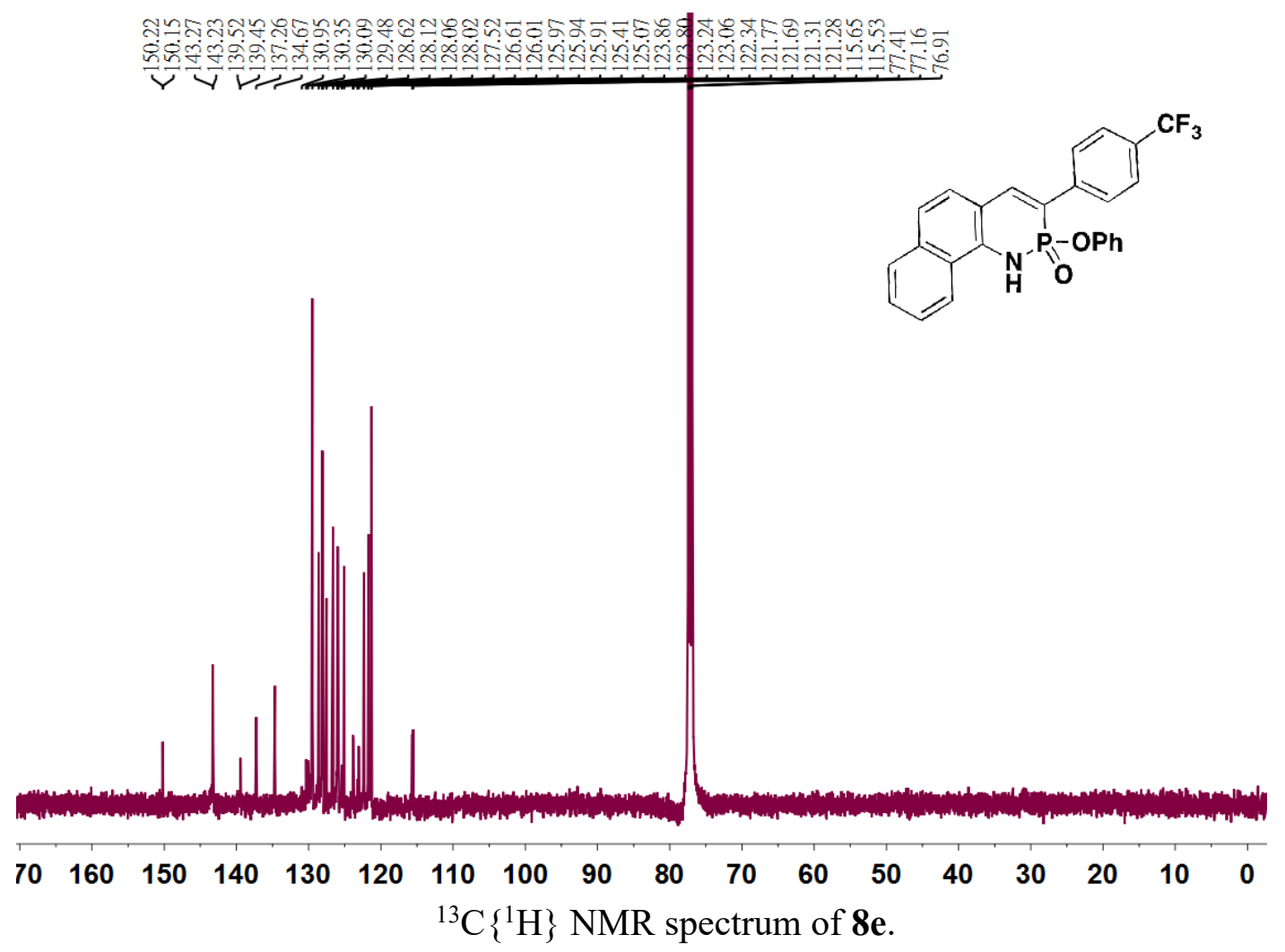

$\frac{\text { đ্d }}{\mathrm{J}}$<smiles>O=P1(O)Nc2c(ccc3ccccc23)C=C1c1ccc(C(F)(F)F)cc1</smiles>

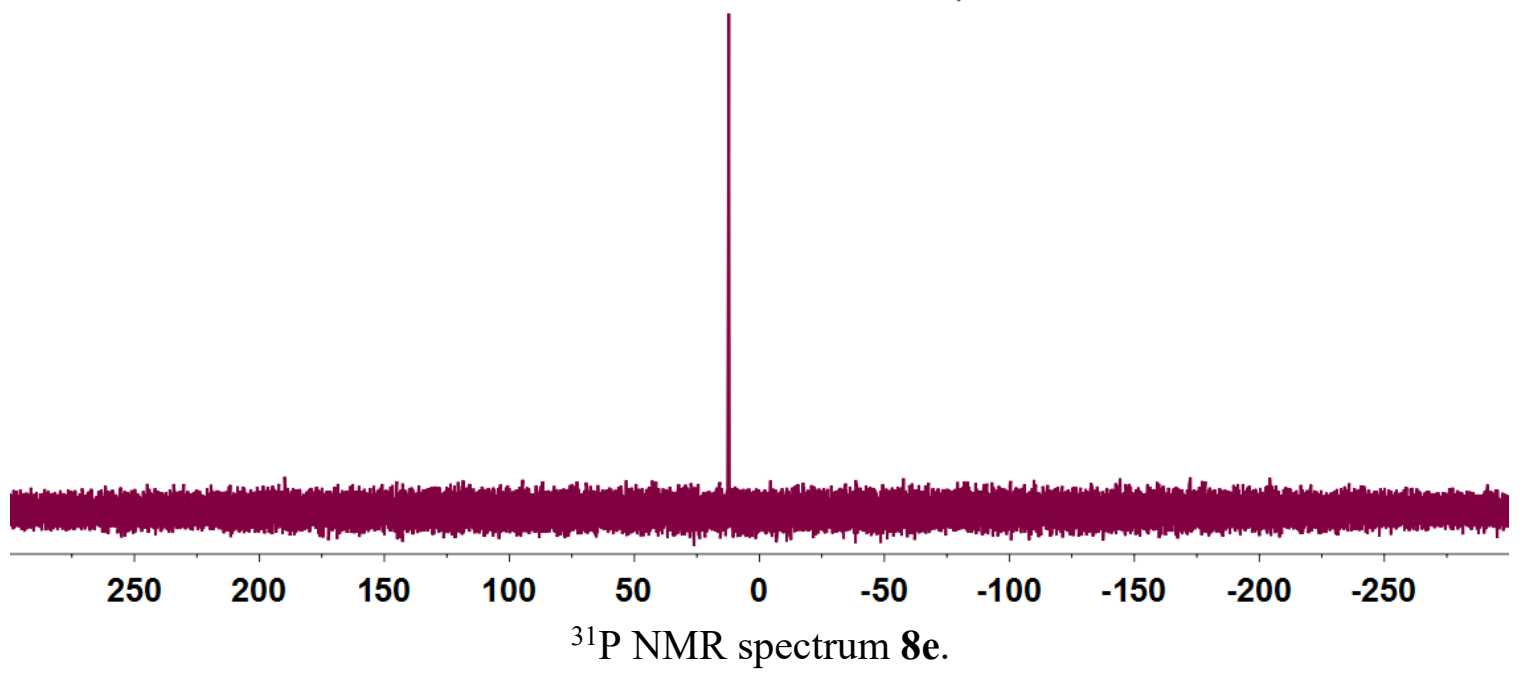



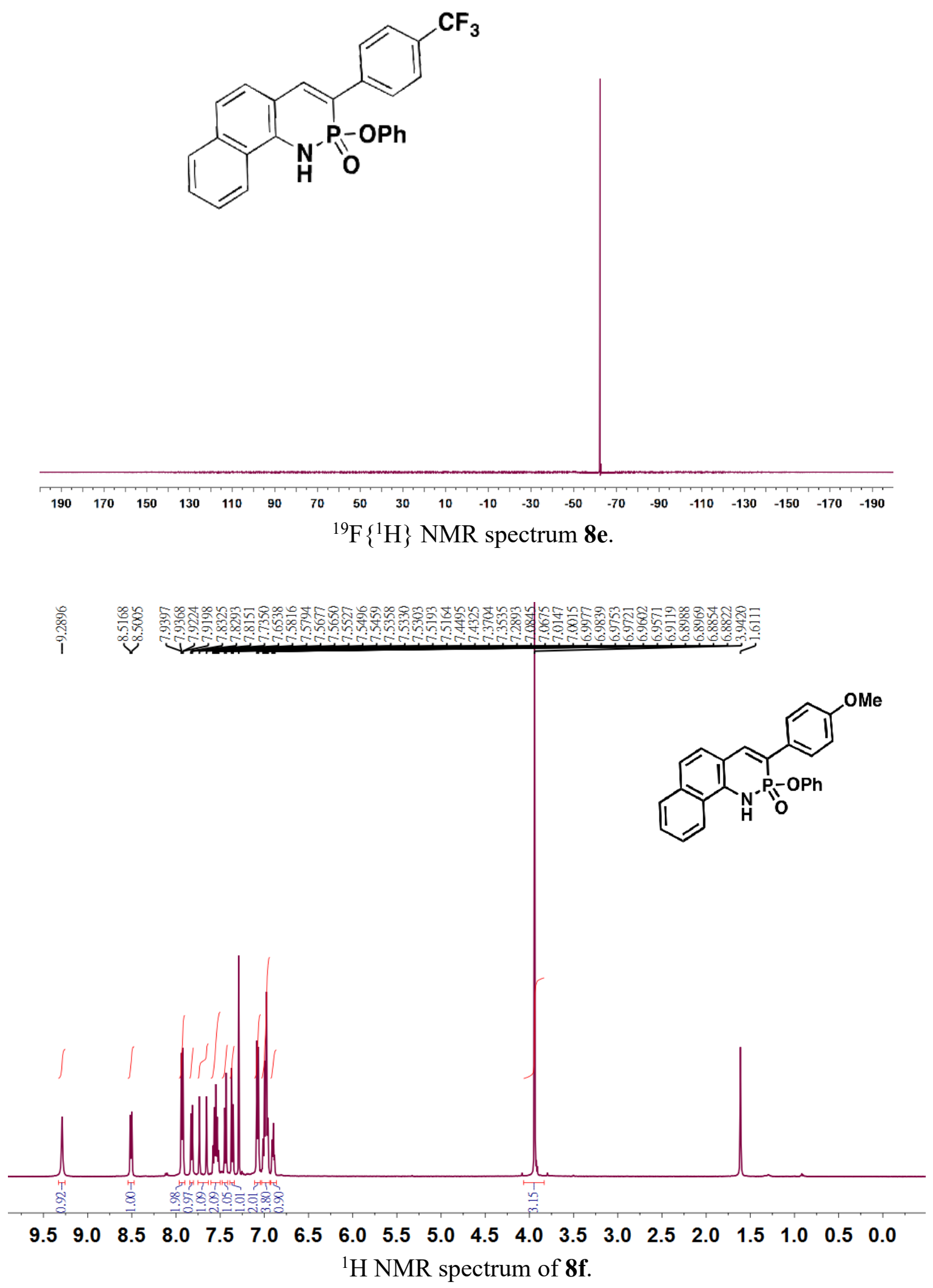


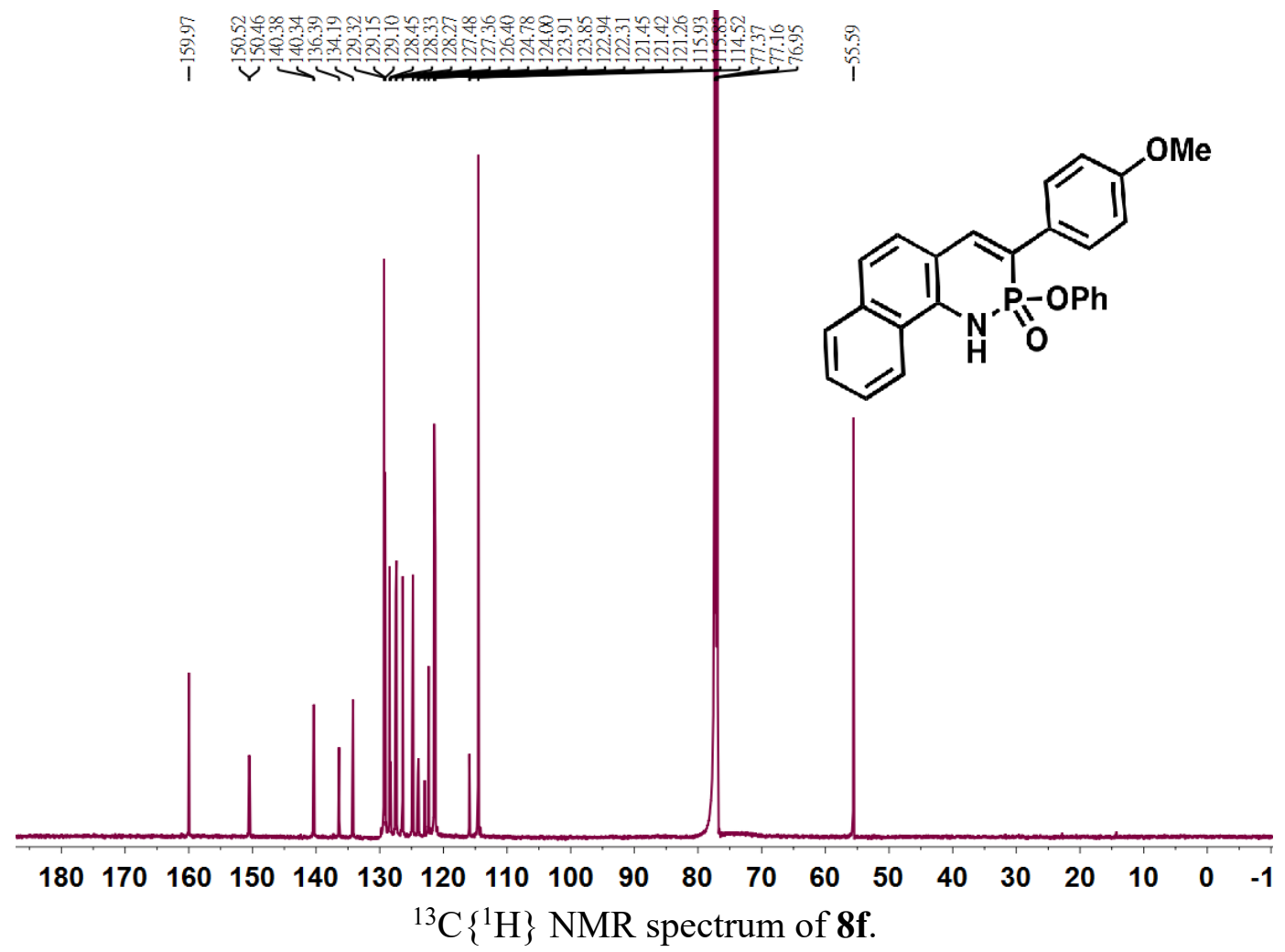

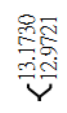<smiles>COc1ccc(C2=Cc3ccc4ccccc4c3NP2(=O)O)cc1</smiles>

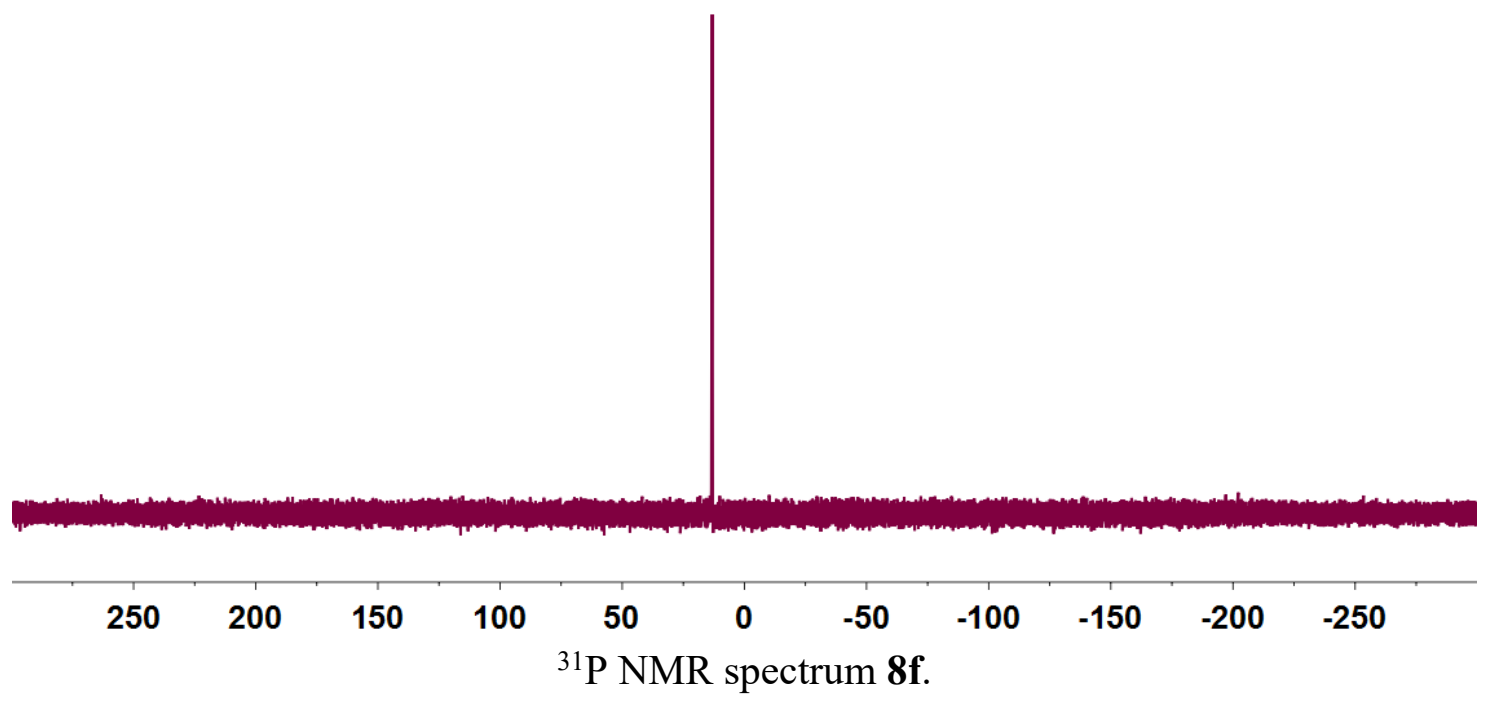

